\title{
Temperature structures in Galactic center clouds
}

\section{Direct evidence for gas heating via turbulence ${ }^{\star}$}

\author{
K. Immer ${ }^{1}$, J. Kauffmann ${ }^{2}$, T. Pillai ${ }^{2}$, A. Ginsburg ${ }^{1}$, and K. M. Menten ${ }^{2}$ \\ ${ }^{1}$ European Southern Observatory, Karl-Schwarzschild-Strasse 2, 85748 Garching bei München, Germany \\ e-mail: ksimmer09@gmail.com \\ 2 Max-Planck-Institut für Radioastronomie, Auf dem Hügel 69, 53121 Bonn, Germany \\ Received 22 April 2016 / Accepted 5 July 2016
}

\begin{abstract}
The central molecular zone at the center of our Galaxy is the best template to study star formation processes under extreme conditions, similar to those in high-redshift galaxies. We observed on-the-fly maps of para- $\mathrm{H}_{2} \mathrm{CO}$ transitions at $218 \mathrm{GHz}$ and $291 \mathrm{GHz}$ towards seven Galactic center clouds. From the temperature-sensitive integrated intensity line ratios of $\mathrm{H}_{2} \mathrm{CO}\left(3_{2,1}-2_{2,0}\right) / \mathrm{H}_{2} \mathrm{CO}\left(3_{0,3}-2_{0,2}\right)$ and $\mathrm{H}_{2} \mathrm{CO}\left(4_{2,2}-3_{2,1}\right) / \mathrm{H}_{2} \mathrm{CO}\left(4_{0,4}-3_{0,3}\right)$ in combination with radiative transfer models, we produce gas temperature maps of our targets. These transitions are sensitive to gas with densities of $\sim 10^{5} \mathrm{~cm}^{-3}$ and temperatures $<150 \mathrm{~K}$. The measured gas temperatures in our sources are all higher $(>40 \mathrm{~K})$ than their dust temperatures $(\sim 25 \mathrm{~K})$. Our targets have a complex velocity structure that requires a careful disentanglement of the different components. We produce temperature maps for each of the velocity components and show that the temperatures of the components differ, revealing temperature gradients in the clouds. Combining the temperature measurements with the integrated intensity line ratio of $\mathrm{H}_{2} \mathrm{CO}\left(4_{0,4}-3_{0,3}\right) / \mathrm{H}_{2} \mathrm{CO}\left(3_{0,3}-2_{0,2}\right)$, we constrain the density of this warm gas to $10^{4}-10^{6} \mathrm{~cm}^{-3}$. We find a positive correlation between the line width of the main $\mathrm{H}_{2} \mathrm{CO}$ lines and the temperature of the gas, direct evidence for gas heating via turbulence. Our data is consistent with a turbulence heating model with a density of $n=10^{5} \mathrm{~cm}^{-3}$.
\end{abstract}

Key words. Galaxy: center - ISM: molecules - ISM: structure - ISM: clouds - submillimeter: ISM

\section{Introduction}

The central region of the Milky Way, the so-called central molecular zone (CMZ), is an exceptional star-forming environment. This region contains $\sim 10 \%$ of the Galaxy's total molecular gas and produces 5-10\% of the Galaxy's infrared and Lyman continuum luminosity (Morris \& Serabyn 1996). The conditions (pressure, magnetic field strength, turbulence, gas temperature, etc.) in this region are much more extreme than in Galactic plane clouds (Morris \& Serabyn 1996). The star formation rate in the CMZ is a factor of 10-100 lower than expected for the huge amount of dense gas and dust contained in this region (e.g. Yusef-Zadeh et al. 2009; Immer et al. 2012; Longmore et al. 2013; Kruijssen \& Longmore 2013).

The high gas temperatures are one of the key properties of the CMZ clouds, influencing the chemistry of the gas, as well as the star formation efficiency of the clouds. It determines the thermal Jeans mass as well as the sound speed that in turn sets the Mach number. Understanding the gas temperature structure of Galactic center clouds is thus crucial for understanding the fragmentation and star forming mechanisms within them. The discrepancy between observed dust and gas temperatures is a long-known feature of CMZ clouds. While multi-wavelength observations of the dust emission in the CMZ yield dust

\footnotetext{
* Integrated intensity maps of the $\mathrm{H}_{2} \mathrm{CO}$ lines (Figs. E.1-E.7), ratio and uncertainty maps (Figs. F.1-F.7), and temperature and uncertainty maps (Figs. H.1-H.7) are only available at the CDS via anonymous ftp to cdsarc.u-strasbg. fr (130.79.128.5) or via http://cdsarc.u-strasbg.fr/viz-bin/qcat?]/A+A/595/A94
}

temperatures of $\sim 20 \mathrm{~K}$ (Lis et al. 1999; Molinari et al. 2011), comparable to the dust temperatures of Galactic plane clouds, the gas temperatures are much higher $(>50 \mathrm{~K}$, Güsten et al. 1981; Hüttemeister et al. 1993; Ao et al. 2013; Mills \& Morris 2013; Ott et al. 2014; Ginsburg et al. 2016). Many previous gas temperature measurements in the CMZ are based on observations of the $\mathrm{NH}_{3}$ molecule which traces low-density gas $\left(n \sim 10^{3} \mathrm{~cm}^{-3}\right.$, e.g. Güsten et al. 1981; Hüttemeister et al. 1993; Ott et al. 2014). Ao et al. (2013) mapped the inner $\sim 75$ pc of the $\mathrm{CMZ}$ in the para- $\mathrm{H}_{2} \mathrm{CO}$ transitions at $218 \mathrm{GHz}$, sensitive to warmer $(T>20 \mathrm{~K})$ and denser $\left(n \sim 10^{4}-10^{5} \mathrm{~cm}^{-3}\right)$ gas. Their results show high gas temperatures towards many CMZ clouds, comparable to those measured in prior studies. This survey was extended to the whole $\mathrm{CMZ}\left(-0.4^{\circ}<l<1.6^{\circ}\right)$ by Ginsburg et al. (2016). The inferred gas temperatures range from $\sim 60 \mathrm{~K}$ to $>100 \mathrm{~K}$, where the highest values are measured towards Sgr B2, the 20 and $50 \mathrm{~km} \mathrm{~s}^{-1}$ clouds, and G0.253+0.016 (The Brick). Comparing their results with dust temperature measurements of the whole CMZ, they show that the gas is uniformly hotter than the dust. The high gas temperatures are consistent with heating through turbulence, while uniform cosmic ray heating is excluded as a dominant heating mechanism.

$\mathrm{H}_{2} \mathrm{CO}$ is a slightly asymmetric rotor molecule. It has two different species (i.e. ortho and para) for which the $K_{\mathrm{a}}{ }^{1}$ quantum number is odd or even. These two species are not connected by radiative transitions. The differences in the population of levels separated by $\Delta K_{\mathrm{a}}=2 \cdot n$ are due to collisions.

\footnotetext{
${ }^{1} K_{\mathrm{a}}$ is the projection of $J$ along the symmetry axis for the limiting case of a prolate (oblate) top.
} 
Table 1. Coordinates and sizes of observed OTF maps at 218 and $291 \mathrm{GHz}$, as well as velocity ranges over which the $\mathrm{H}_{2} \mathrm{CO}$ line emission was integrated (see Sect 3.1).

\begin{tabular}{|c|c|c|c|c|c|c|c|c|c|}
\hline \multirow[t]{2}{*}{ Source } & \multirow{2}{*}{$\begin{array}{c}\text { RA } \\
\text { (hh mm ss.ss) } \\
\text { (EQ 2000) }\end{array}$} & \multirow{2}{*}{$\begin{array}{c}\text { Dec } \\
(\mathrm{dd} " \prime \prime \prime \prime \prime . ") \\
(\text { EQ 2000) }\end{array}$} & \multirow[t]{2}{*}{$\begin{array}{c}v_{\mathrm{LSR}} \\
\left(\mathrm{km} \mathrm{s}^{-1}\right)\end{array}$} & \multicolumn{2}{|c|}{$\begin{array}{l}\text { OTF size } \\
\left({ }^{\prime \prime} x^{\prime \prime}\right)\end{array}$} & & \\
\hline & & & & $218 \mathrm{GHz}$ & $291 \mathrm{GHz}$ & $218 \mathrm{GHz}$ & $291 \mathrm{GHz}$ & Whole Source & Vel. Comp. \\
\hline $20 \mathrm{~km} \mathrm{~s}^{-1}$ cloud & 174536.89 & -290512.0 & 20 & $115 \times 200$ & $180 \times 270$ & 41 & 30 & $-15-36$ & $\begin{array}{c}0-6 \\
8-14 \\
27-33\end{array}$ \\
\hline $50 \mathrm{~km} \mathrm{~s}^{-1}$ cloud & 174551.18 & -285934.3 & 50 & $115 \times 150$ & $180 \times 180$ & 59 & 46 & $16-80$ & $\begin{array}{c}41-47 \\
57-63 \\
-3-3\end{array}$ \\
\hline $\mathrm{G} 0.253+0.016$ & 174608.55 & -284244.0 & 25 & $115 \times 200$ & $200 \times 340$ & 35 & 44 & $-6-54$ & $\begin{array}{l}16-22 \\
36-42 \\
75-81\end{array}$ \\
\hline $\mathrm{G} 0.411+0.050$ & 174624.44 & -283326.6 & 25 & $115 \times 115$ & $180 \times 240$ & 36 & 44 & $10-30$ & $19-25$ \\
\hline G0.480 & 1746 & -283 & 25 & $115 \times 115$ & $180 \times$ & 49 & 43 & & $27-33$ \\
\hline Sgr C & 1744 & -292753.7 & -50 & & $200 \times 180$ & & 44 & $-60--46$ & $-55--49$ \\
\hline Sgr D & 174841.75 & -280145.0 & -15 & & $180 \times 180$ & & 44 & $-17--14$ & $-19--13$ \\
\hline
\end{tabular}

Notes. Columns 6 and 7 give the median rms values of the $\mathrm{H}_{2} \mathrm{CO}$ cubes of the whole velocity range of each source at 218 and $291 \mathrm{GHz}$.

Mangum \& Wootten (1993) presented a detailed study of the usability of different $\mathrm{H}_{2} \mathrm{CO}$ transitions for the determination of the kinetic temperature and density of the gas in molecular clouds. For a range of total angular momentum quantum numbers $J$ (here $J=2$ and 3), modeling of the relative intensities of (in our case) para- $\mathrm{H}_{2} \mathrm{CO}$ lines ( $K_{\mathrm{a}}$ quantum number of 0 or 2 ), delivers estimates of the density and the temperature. The $K_{\mathrm{a}}$ ladders are close in frequency and thus can be observed with the same telescope, even very often in the same spectrum, which makes them calibration-independent.

The $J=3-2$ and 4-3 $\mathrm{H}_{2} \mathrm{CO} K_{\mathrm{a}}$ ladders at 218 and $291 \mathrm{GHz}$, respectively, can be observed with the Atacama Pathfinder Experiment (APEX) telescope, each group within one band. Mangum \& Wootten (1993) showed that measuring several $\mathrm{H}_{2} \mathrm{CO}$ intensity ratios of transitions with different $J$ values yields better constraints of the kinetic temperature than can be obtained from just one $\mathrm{H}_{2} \mathrm{CO}$ intensity ratio. This is clear since then a larger range of level energies is covered.

In this paper, we report a detailed gas temperature study of seven molecular clouds in the CMZ, using the $\mathrm{H}_{2} \mathrm{CO}$ thermometer at 218 and $291 \mathrm{GHz}$. The names of the observed sources and their coordinates are listed in Table 1. We chose our targets to be high density clouds with previous warm gas temperature measurements. As shown in Fig. 1, they span the whole CMZ. None of these clouds are photon-dominated or $\mathrm{X}$-ray-dominated regions. There is evidence of wide-spread shocks in the form of $\mathrm{SiO}$ emission in the $\mathrm{CMZ}$ including these clouds. Our sample contains potential star-forming clouds (Sgr C, $20 \mathrm{~km} \mathrm{~s}^{-1}$ cloud, $50 \mathrm{~km} \mathrm{~s}^{-1}$ cloud, G0.4800.006), quiescent clouds $(\mathrm{G} 0.411+0.050)$ and shock-heated clouds (G0.253+0.016). There is an ongoing debate whether Sgr $\mathrm{D}$ is part of the CMZ or not (Mehringer et al. 1998; Blum et al. 1999; Sawada et al. 2009. However, this uncertainty does not influence our results or conclusions.

In Sect. 2, we describe the observations and the calibration of the data. In Sect. 3, we present how the $\mathrm{H}_{2} \mathrm{CO}$ spectra, ratio, and uncertainty maps were produced. In Sect. 4 , the radiative transfer modeling is described. We discuss the different results in Sect. 5 and give conclusions in Sect. 6.

\section{Observation and data reduction}

\subsection{Observations}

In 2012 and 2014, we observed five and seven molecular clouds in the CMZ (Table 1) with the $\mathrm{APEX}^{2}$ telescope (Güsten et al. 2006) at 218 and $291 \mathrm{GHz}$, respectively (project codes: M-089.F-0029-2012, M-093.F-0030-2014). On-thefly (OTF) maps were taken with the Swedish heterodyne facility instrument ( $\mathrm{SHeFi}$, Vassilev et al. 2008), as well as the First Light APEX Submillimeter Heterodyne (FLASH) receiver (Heyminck et al. 2006; Klein et al. 2014), using the eXtended bandwidth Fast Fourier Transform Spectrometer (XFFTS) backend (Klein et al. 2012). Table 2 shows the covered para- $\mathrm{H}_{2} \mathrm{CO}$ transitions.

The SHeFi observations were centered at $218.9 \mathrm{GHz}$ and covered a bandwidth of $4 \mathrm{GHz}$. The FLASH observations were taken in the frequency ranges $278.5-282.5 \mathrm{GHz}$ and 290.5-294.5 GHz. The XFFTS backend provides a fixed number of 32768 spectral channels, resulting in a resolution of $0.1 \mathrm{~km} \mathrm{~s}^{-1}$ at $218 \mathrm{GHz}$ and $0.04 \mathrm{~km} \mathrm{~s}^{-1}$ at $291 \mathrm{GHz}$. To increase the signal-to-noise ratio of the spectra, we smoothed the two datasets by a factor of 9 and 24, respectively, yielding a velocity resolution of $\sim 0.9 \mathrm{~km} \mathrm{~s}^{-1}$. Since we do not expect to detect lines in our spectra that are narrower than a few $\mathrm{km} \mathrm{s}^{-1}$, this velocity resolution is sufficient. The full widths at half maximum of the beams were $30^{\prime \prime}$ at $218 \mathrm{GHz}$ and $24^{\prime \prime}$ at $291 \mathrm{GHz}$.

A first calibration of the data was conducted already at the telescope, yielding spectra with calibrated fluxes at each position of the OTF maps. The calibration error of this step is about $15 \%$ for each dataset (SHeFI calibration plan; A. Belloche for FLASH, priv. comm.). We then converted the antenna temperatures of the spectra into main beam temperatures, using Ruze's equation with the scaling factor being 0.69 and the width factor 19 micron. The two datasets were further edited and analyzed with the software CLASS from the GILDAS package (Pety 2005).

2 This publication is based on data acquired with the Atacama Pathfinder Experiment (APEX). APEX is a collaboration between the Max-Planck-Institut für Radioastronomie, the European Southern Observatory, and the Onsala Space Observatory. 


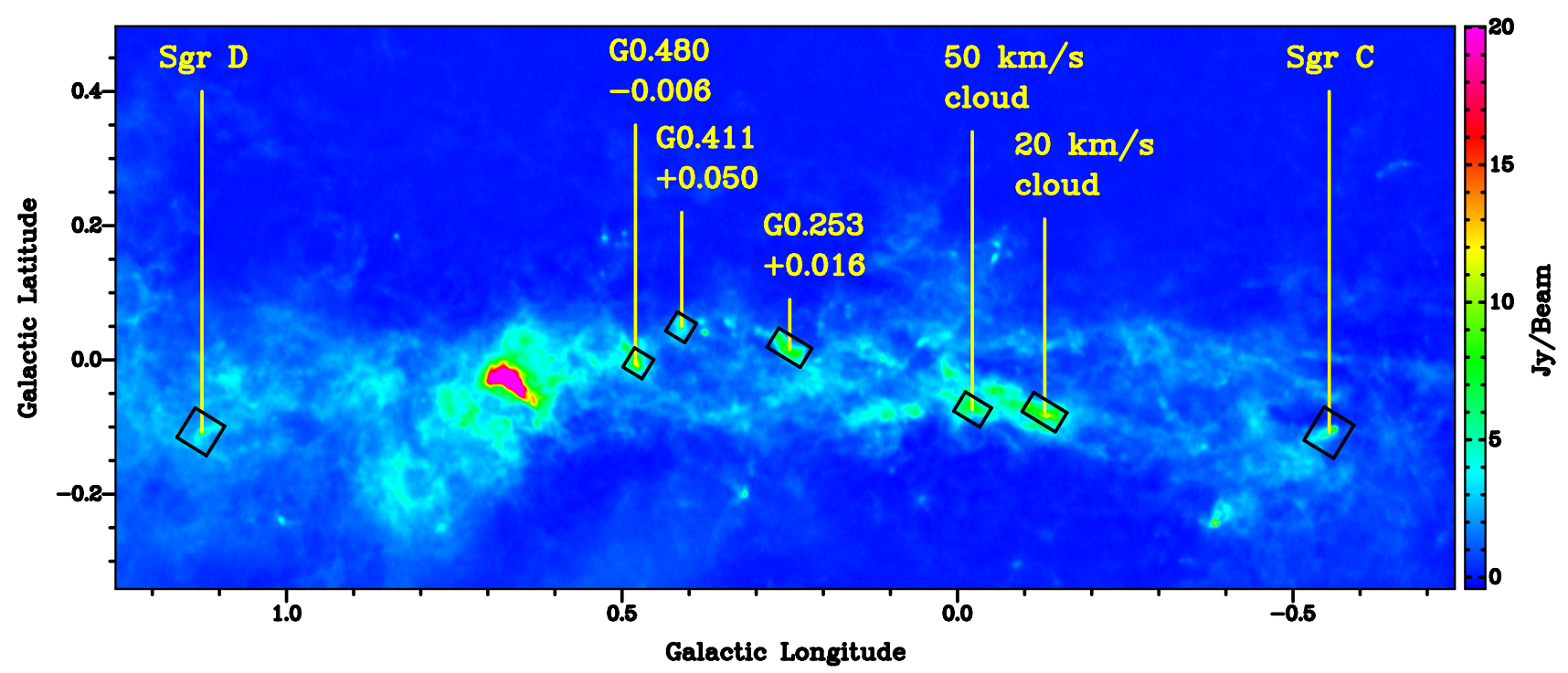

Fig. 1. $870 \mu \mathrm{m}$ emission of the CMZ from the ATLASGAL survey (Schuller et al. 2009). The targets of our temperature study are marked. The boxes show the sizes of the observed OTF maps at $218 \mathrm{GHz}$ for the 20 and $50 \mathrm{~km} \mathrm{~s}^{-1}$ clouds, G0.253+0.016, G0.411+0.050, and G0.480-0.006, and at $291 \mathrm{GHz}$ for Sgr C and Sgr D.

Table 2. Properties of covered para- $\mathrm{H}_{2} \mathrm{CO}$ transitions.

\begin{tabular}{ccc}
\hline \hline Transition & $\begin{array}{c}v \\
(\mathrm{GHz})\end{array}$ & $\begin{array}{c}E_{\mathrm{up}} \\
(\mathrm{K})\end{array}$ \\
\hline $3_{0,3}-2_{0,2}$ & 218.22219 & 20.96 \\
$3_{2,2}-2_{2,1}$ & 218.47563 & 68.09 \\
$3_{2,1}-2_{2,0}$ & 218.76007 & 68.11 \\
\hline $4_{0,4}-3_{0,3}$ & 290.62341 & 34.90 \\
$4_{2,3}-3_{2,2}$ & 291.23778 & 82.07 \\
$4_{2,2}-3_{2,1}$ & 291.94806 & 82.12 \\
\hline
\end{tabular}

Notes. Columns give quantum numbers, $\mathrm{J}_{\mathrm{K}_{\mathrm{a}}, \mathrm{K}_{\mathrm{c}}}$, frequencies and level energies above the ground state. The critical densities of $3_{0,3}-2_{0,2}$ and $4_{0,4}-3_{0,3}$ are $\sim 6 \times 10^{5} \mathrm{~cm}^{-3}$ and $\sim 1 \times 10^{6} \mathrm{~cm}^{-3}$, respectively (Shirley 2015).

\subsection{Baseline subtraction}

The $218 \mathrm{GHz}$ spectra were strongly affected by bad baselines (Fig. A.1). Since the baselines could not be cleanly removed by fitting low-level polynomials to the data, we fitted the spectra with splines over the velocity ranges $-90-250 \mathrm{~km} \mathrm{~s}^{-1}$ for the $20 \mathrm{~km} \mathrm{~s}^{-1}$ cloud, G0.253+0.016, G0.411+0.050, and G0.480-0.006, and $-60-220 \mathrm{~km} \mathrm{~s}^{-1}$ for the $50 \mathrm{~km} \mathrm{~s}^{-1}$ cloud. First, the raw data were smoothed to a velocity resolution of $\sim 0.9 \mathrm{~km} \mathrm{~s}^{-1}$, then lines in the spectra were fitted with Gaussians and removed from the spectra if they were at least a $3 \sigma$ detection. Then, the data were downsampled to a resolution of $\sim 36 \mathrm{~km} \mathrm{~s}^{-1}$ to ensure that weak line emission that was not removed in the previous step would be smoothed out over the adjacent channels. This spectrum was then resampled to the original resolution of $0.9 \mathrm{~km} \mathrm{~s}^{-1}$, yielding the spline spectrum, and then subtracted from the input spectrum. However, in some of the spectra, the baseline features are as narrow as the spectral lines and could not be removed completely, resulting in negative features in the final spectra. Figure A.1 shows one input spectrum, the fitted spline, and the final spectrum. The gray box marks one of the narrow negative features in the spectrum.
The $291 \mathrm{GHz}$ dataset was much less affected by bad baselines. The baselines were removed by fitting each spectrum with a 1st-order polynomial over the velocity ranges -120 $120 \mathrm{~km} \mathrm{~s}^{-1}$ (20 km s${ }^{-1}$ cloud), $-70-120 \mathrm{~km} \mathrm{~s}^{-1}$ (50 $\mathrm{km} \mathrm{s}^{-1}$ cloud), -90-120 $\mathrm{km} \mathrm{s}^{-1}$ (G0.253+0.016, G0.411+0.050, G0.480-0.006), and -200-100 $\mathrm{km} \mathrm{s}^{-1}$ (Sgr C, Sgr D).

\section{3. $\mathrm{H}_{2} \mathrm{CO}$ spectra}

To obtain average spectra of the six $\mathrm{H}_{2} \mathrm{CO}$ lines at $218 \mathrm{GHz}$ and $291 \mathrm{GHz}$, we averaged the 218.15-218.85 GHz and 290.55-292.1 GHz emission, respectively, over the OTF map of each source. As an example, the spectra of the $20 \mathrm{~km} \mathrm{~s}^{-1}$ cloud and G0.411+0.050 are shown in Fig. 2. In addition to the six $\mathrm{p}-\mathrm{H}_{2} \mathrm{CO}$ lines in the two frequency ranges, we marked the transitions $\mathrm{HC}_{3} \mathrm{~N}(24-23), \mathrm{CH}_{3} \mathrm{OH}\left(4_{2,2}-3_{1,2}\right)$, and $\mathrm{o}-\mathrm{H}_{2} \mathrm{CO}\left(4_{3,2}-3_{3,1}\right)$. The spectra of all sources are presented in Fig. B.1. To better compare the strength of the different lines, we chose the same intensity scale for all spectra.

The $\mathrm{H}_{2} \mathrm{CO}$ emission at both frequencies is strongest in the 20 and $50 \mathrm{~km} \mathrm{~s}^{-1}$ clouds. Here, all six $\mathrm{p}-\mathrm{H}_{2} \mathrm{CO}$ lines are welldetected. In $\mathrm{G} 0.411+0.050$ and $\mathrm{Sgr} \mathrm{D}$, the $\mathrm{H}_{2} \mathrm{CO}$ emission at $291 \mathrm{GHz}$ is almost not detected in the average spectra.

In Fig. B.2, we overplot the $\mathrm{H}_{2} \mathrm{CO}\left(3_{0,3}-2_{0,2}\right)$ and the $\mathrm{H}_{2} \mathrm{CO}\left(4_{0,4}-3_{0,3}\right)$ spectra for the 20 and $50 \mathrm{~km} \mathrm{~s}^{-1}$ clouds. The figure shows that the line width of the two transitions is comparable.

\subsection{Methanol contamination}

The $\mathrm{H}_{2} \mathrm{CO}\left(3_{2,2}-2_{2,1}\right)$ and the $\mathrm{CH}_{3} \mathrm{OH}\left(4_{2,2}-3_{1,2}\right)$ transitions are separated by $35 \mathrm{MHz}$, corresponding to $25.5 \mathrm{~km} \mathrm{~s}^{-1}$. These two lines are well-resolved and well-separated in our data. However, in sources for which the range of velocity components extends over more than $26 \mathrm{~km} \mathrm{~s}^{-1}$, the integrated intensity maps of the $\mathrm{H}_{2} \mathrm{CO}\left(3_{2,2}-2_{2,1}\right)$ line are contaminated by methanol emission for certain velocity components (see Fig. C.1). We thus excluded this line from the further analysis. 

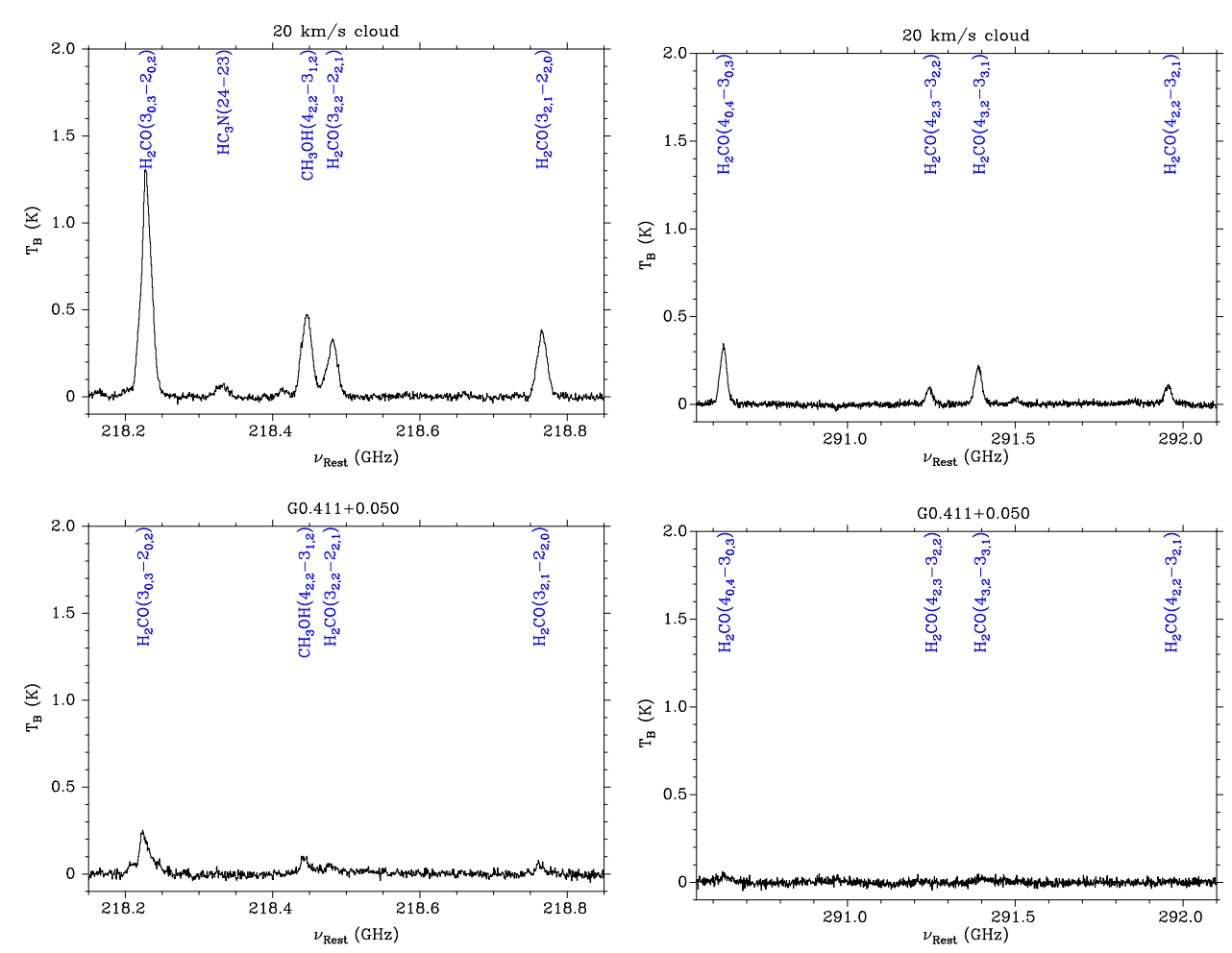

Fig. 2. Spectra of para- $\mathrm{H}_{2} \mathrm{CO}$ transitions at 218 (left panels) and $291 \mathrm{GHz}$ (right panels) of the $20 \mathrm{~km} \mathrm{~s}^{-1}$ cloud (upper panels) and G0.411+0.050 (lower panels), averaged over the whole OTF maps. Transitions of $\mathrm{H}_{2} \mathrm{CO}$, as well as other molecules are marked in the spectra.

\section{Analysis}

\subsection{Velocity ranges}

To determine the whole velocity range covered by $\mathrm{H}_{2} \mathrm{CO}$ emission for each source, we used the $218 \mathrm{GHz}$ spectra (Fig. B.1, Sect. 2.3) and zoomed in on the $\mathrm{H}_{2} \mathrm{CO}\left(3_{0,3}-2_{0,2}\right)$ line. We fitted a linear baseline to line-free channels close to the line to obtain the average rms level in each OTF map. For each source, we determined the velocity range where the line emission was above $3 \sigma$ (Col. 9 in Table 1, Fig. D.1). For Sgr C and Sgr D, we followed the same procedure but used the transition $\mathrm{H}_{2} \mathrm{CO}\left(4_{0,4}-3_{0,3}\right)$.

To detect different velocity components, we subdivided the OTF maps into small tiles of size $33^{\prime \prime} \times 33^{\prime \prime}$, slightly larger than the beam, and averaged the $\mathrm{H}_{2} \mathrm{CO}\left(3_{0,3}-2_{0,2}\right)$ spectra over these areas. We fitted the line with several Gaussians if more than one velocity component was present and determined the central velocity of each component in each tile.

For each velocity component in each source, we averaged the $\mathrm{H}_{2} \mathrm{CO}\left(3_{0,3}-2_{0,2}\right)$ spectra $\left(\mathrm{H}_{2} \mathrm{CO}\left(4_{0,4}-3_{0,3}\right)\right.$ in the case of Sgr C and Sgr D) over the area where this component dominated and again estimated the central velocity of the line in the averaged spectrum. To separate the components well, spatially and spectrally, in the following analysis, we set the velocity ranges over which the $\mathrm{H}_{2} \mathrm{CO}$ emission is integrated as $\pm 3 \mathrm{~km} \mathrm{~s}^{-1}$ around the central velocity of each component (Col. 10 in Table 1).

We detected velocity gradients in three of our clouds. In $\mathrm{G} 0.253+0.016$, the average velocities range from $\sim 40 \mathrm{~km} \mathrm{~s}^{-1}$ in the south-west of the cloud to $\sim 0 \mathrm{~km} \mathrm{~s}^{-1}$ in the North-East. We identified four spatially distinct velocity components at 0,19 , 39 , and $78 \mathrm{~km} \mathrm{~s}^{-1}$ in this source. While the emission of the first three velocity components peaks towards the cloud, the emission of the $78 \mathrm{~km} \mathrm{~s}^{-1}$ component originates from the south-east border of the cloud and might not be associated with this cloud.

In the $20 \mathrm{~km} \mathrm{~s}^{-1}$ cloud, the velocity gradient spreads over $\sim 30 \mathrm{~km} \mathrm{~s}^{-1}$ from the south-east end of the cloud to the northwest part. The three velocity components we identified in this source are at 3,11 , and $30 \mathrm{~km} \mathrm{~s}^{-1}$. Most of the $50 \mathrm{~km} \mathrm{~s}^{-1}$ cloud is at $\sim 45 \mathrm{~km} \mathrm{~s}^{-1}$ but there are higher velocities detected at the north and lower velocities at the west end of the cloud. The velocity gradient also spans about $\sim 15 \mathrm{~km} \mathrm{~s}^{-1}$. We could distinguish between two velocity components at 44 and $60 \mathrm{~km} \mathrm{~s}^{-1}$.

We identified only one velocity component in the other clouds. The $\mathrm{H}_{2} \mathrm{CO}$ emission peaks at $22 \mathrm{~km} \mathrm{~s}^{-1}$ in $\mathrm{G} 0.411+0.050,30 \mathrm{~km} \mathrm{~s}^{-1}$ in G0.480-0.006, $-52 \mathrm{~km} \mathrm{~s}^{-1}$ in Sgr C, and $-16 \mathrm{~km} \mathrm{~s}^{-1}$ in Sgr D.

\subsection{Integrated intensity ratio and uncertainty maps}

For each $\mathrm{H}_{2} \mathrm{CO}$ transition in Table 2, we produced calibrated data cubes on a grid with a resolution of $1 \mathrm{~km} \mathrm{~s}^{-1}$. The cubes are smoothed to a resolution of $33^{\prime \prime}$. The pixel size is $11^{\prime \prime}$. For each pixel of these cubes, we fitted linear baselines to line-free velocities at both sides of the lines. For each line and all velocity components of our targets, we produced maps of the uncertainty of the line intensity by assigning every pixel of the map the corresponding $\sigma\left(T_{\mathrm{mb}}\right)$ from the baseline fitting. The median of $\sigma\left(T_{\mathrm{mb}}\right)$ of the maps ranges between 35 and $59 \mathrm{mK}$ per pixel at $218 \mathrm{GHz}$ and 30 and $46 \mathrm{mK}$ per pixel at $291 \mathrm{GHz}$ (Cols. 7, 8 in Table 1).

As described in Sect. 1, the relative intensities of para- $\mathrm{H}_{2} \mathrm{CO}$ lines yield estimates of the temperature and density of the gas. 
We selected the following key line ratios:

$$
\begin{aligned}
& R_{321}=\frac{\int I_{\mathrm{H}_{2} \mathrm{CO}\left(3_{2,1}-2_{2,0}\right)} \mathrm{d} v}{\int I_{\mathrm{H}_{2} \mathrm{CO}\left(3_{0,3}-2_{0,2}\right)} \mathrm{d} v} \Rightarrow \text { Temperature } \\
& R_{422}=\frac{\int I_{\mathrm{H}_{2} \mathrm{CO}\left(4_{2,2}-3_{2,1}\right)} \mathrm{d} v}{\int I_{\mathrm{H}_{2} \mathrm{CO}\left(4_{0,4}-3_{0,3}\right)} \mathrm{d} v} \Rightarrow \text { Temperature } \\
& R_{404}=\frac{\int I_{\mathrm{H}_{2} \mathrm{CO}\left(4_{0,4}-3_{0,3}\right)} \mathrm{d} v}{\int I_{\mathrm{H}_{2} \mathrm{CO}\left(3_{0,3}-2_{0,2}\right)} \mathrm{d} v} \Rightarrow \text { Density. }
\end{aligned}
$$

For a better readability of the paper, we will abbreviate these ratios to $R_{321}, R_{404}$, and $R_{422}$, respectively, in the following text.

For each pixel in the data cubes, we integrated the emission of the para- $\mathrm{H}_{2} \mathrm{CO}$ lines independently over the same velocity range $v_{\text {range }}$, yielding separate integrated intensity maps for the different lines. We produced these maps for all velocity components of our targets $\left(v_{\text {range }}\right.$ in Cols. 9, 10 in Table 1; Figs. E.1-E.7).

We identified the pixels in the OTF maps of each source where the integrated emission of the $\mathrm{H}_{2} \mathrm{CO}\left(3_{0,3}-2_{0,2}\right)$ and the $\mathrm{H}_{2} \mathrm{CO}\left(4_{0,4}-3_{0,3}\right)$ lines, respectively, was above the $5 \cdot \sigma\left(T_{\mathrm{mb}}\right) \cdot \sqrt{\frac{v_{\text {range }}}{\Delta v_{\text {res }}}} \cdot \Delta v_{\text {res }}$ threshold. The parameter $\Delta v_{\text {res }}$ is the velocity resolution of our data. For these pixels, we determined the aforementioned ratios from the integrated intensity maps of these lines, yielding integrated intensity ratio maps (Figs. F.1-F.7).

For positions where the integrated emission of the line in the numerator was below the $5 \sigma$ threshold, the integrated intensity value was exchanged for the value $5 \cdot \sigma\left(T_{\mathrm{mb}}\right) \cdot \sqrt{\frac{v_{\text {range }}}{\Delta v_{\text {res }}}} \cdot \Delta v_{\text {res }}$, representing upper limits of the ratios (marked with Xs in the maps). For each pixel in the ratio maps (except upper limits), we then determined the uncertainty of the ratio via Gaussian error propagation, using the uncertainty maps of the intensity of the two corresponding lines. The remaining pixels (corresponding to the pixels with upper limits in the ratio maps) were blanked in gray. In two velocity components of two sources, all or almost all pixels are upper limits (G0.253+0.016: 16-22 $\mathrm{km} \mathrm{s}^{-1}, 291 \mathrm{GHz}$; 75-81 $\mathrm{km} \mathrm{s}^{-1}, 218$ and $291 \mathrm{GHz}$; G0.411+0.050: both velocity components, $291 \mathrm{GHz}$ ).

\section{Modeling}

\subsection{Fundamental approach}

We use RADEX (van der Tak et al. 2007) and a related solver (Fujun Du's myRadex ${ }^{3}$ ) to create model intensities for the para- $\mathrm{H}_{2} \mathrm{CO}$ lines. The calculations are executed using pyradex, a Python-based wrapper for RADEX (developed by A. Ginsburg ${ }^{4}$ ). The collision rates used here (from Wiesenfeld \& Faure 2013) are retrieved from the LAMDA database (Schöier et al. 2005). This approach is conceptually identical to the one employed by Ginsburg et al. (2016), though we choose slightly different gas properties to solve the radiative transfer problem.

We adopt a fiducial set of conditions for our calculations of emission line intensities and ratios. Below we show that our temperature estimates are robust with respect to the choice of values for these parameters. We assume $\mathrm{H}_{2}$ particle densities of $10^{5} \mathrm{~cm}^{-3}$. This choice is motivated by the average densities

\footnotetext{
https://github.com/fjdu/myRadex

4 https://github.com/keflavich/pyradex
}

of a few $10^{4} \mathrm{~cm}^{-3}$ as estimated for our targets via the analysis of dust emission maps (Kauffmann et al. 2016). The column density of para- $\mathrm{H}_{2} \mathrm{CO}$ is set to $5 \times 10^{13} \mathrm{~cm}^{-2}$. For a representative $\mathrm{H}_{2}$ column density of $5 \times 10^{22} \mathrm{~cm}^{-2}$ (Kauffmann et al. 2016) this corresponds to an abundance of $10^{-9}$ with respect to $\mathrm{H}_{2}$, a value that is suggested by previous work (for a summary, see Ginsburg et al. 2016). We adopt a velocity dispersion of $5 \mathrm{~km} \mathrm{~s}^{-1}$. This value is appropriate for structures with a radius of order $1 \mathrm{pc}$, which is about the diameter of our beam. The calculations are executed assuming a spherical geometry.

We compute the intensities of para- $\mathrm{H}_{2} \mathrm{CO}$ emission lines under the aforementioned conditions. These intensities can be used to calculate the $R_{321}$ and $R_{422}$ line ratios. These line ratios are known to be highly sensitive to the temperature while being rather insensitive to changes in other parameters (e.g. Mangum \& Wootten 1993, see left panel of Fig. 3 for $R_{321}$ ). This trend is confirmed in calculations discussed below. For this reason, the $R_{321}$ and $R_{422}$ line ratios can be used to estimate the kinetic gas temperature.

In essence, we use the aforementioned RADEX calculations for kinetic gas temperatures of 10 to $300 \mathrm{~K}$ to obtain functions that yield estimates of the kinetic gas temperature for given $R_{321}$ or $R_{422}$. The ratios range from 0 to values larger than 0.5 for this temperature range. The resulting function for $R_{321}$ is almost identical to the one used by Ginsburg et al. (2016). This is in particularly remarkable because Ginsburg et al. use large velocity gradient (LVG) calculations, while we choose to adopt spherical model geometries. Here, we opt for the latter modeling geometry since it is close to the naive picture of a cloud with a well-defined boundary that is immersed in tenuous material. The comparison with the LVG case shows, however, that our analysis is robust with respect to the adopted modeling geometry.

\subsection{Systematic uncertainties}

We assess the robustness of this method by repeating the line ratio calculations for different choices in gas properties. We vary the velocity dispersion between 2 and $10 \mathrm{~km} \mathrm{~s}^{-1}$, increase and decrease in column density by a factor of 10 , explore lower densities of $10^{4} \mathrm{~cm}^{-3}$, and examine the results for the slab and LVG models available within RADEX (Fig. G.1). This shows that, for a given line ratio, none of these modifications lead to temperatures that deviate by more than $\pm 30 \%$ from the result obtained using our fiducial case (see Fig. G.1). For reference, we note that spherical model geometries give the lowest temperatures, followed by slightly elevated values for LVG conditions. Slab geometries, however, give, for a fixed line ratio, temperatures that are up to $30 \%$ higher than those found for the fiducial case. We note in particular that these calculations explore the density range between $10^{4}$ and $10^{5} \mathrm{~cm}^{-3}$ that is suggested by the observations of dust emission discussed by Kauffmann et al. (2016). Variations in density by a factor 10 have no significant impact on our results.

In the high column density Galactic center environment, the $\mathrm{H}_{2} \mathrm{CO}\left(3_{0,3}-2_{0,2}\right)$ emission might be optically thick. This saturation would cause a falsely high $R_{321}$ line ratio in our integrated intensity ratio maps. A higher ratio implies higher temperatures. Thus, an optically thick $\mathrm{H}_{2} \mathrm{CO}\left(3_{0,3}-2_{0,2}\right)$ line would mimic higher gas temperatures in the clouds.

We have executed more detailed experiments for selected positions to understand the impact of the line optical depth. For example, we modeled the emission from the $20 \mathrm{~km} \mathrm{~s}^{-1}$ cloud in the velocity slice of 8 to $14 \mathrm{~km} \mathrm{~s}^{-1}$ (Fig. G.2, left panel). The $3_{0,3}-2_{0,2}$ transition has a velocity-integrated peak intensity of 

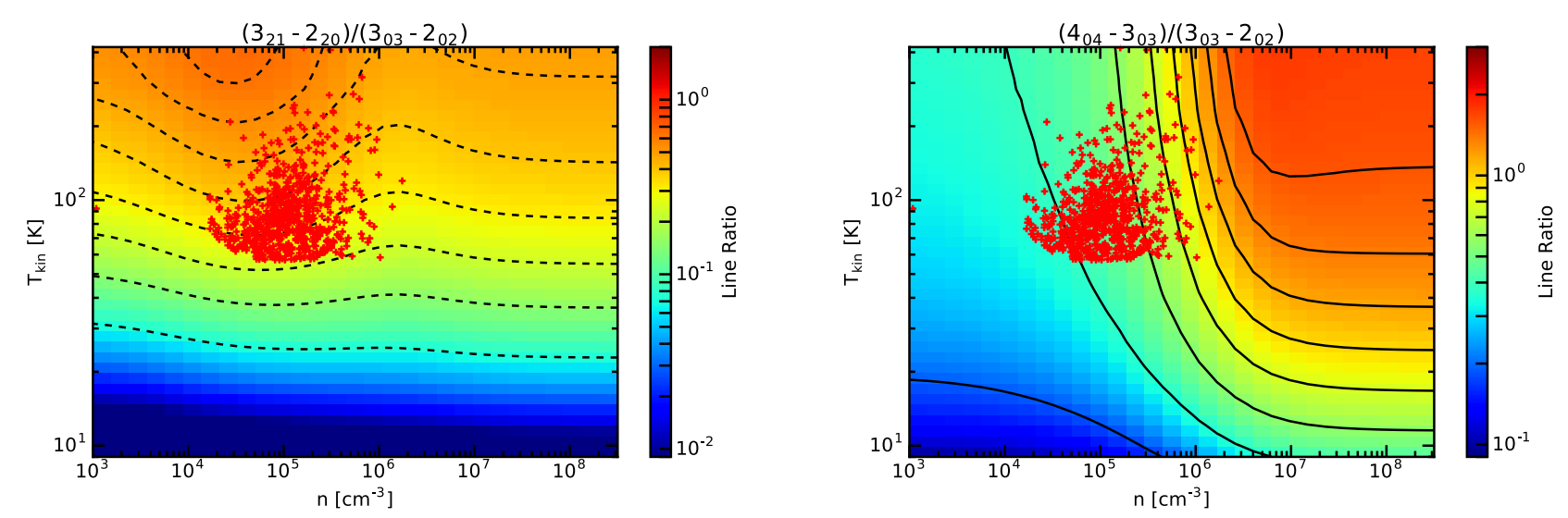

Fig. 3. Left: $R_{321}$ as a function of kinetic temperature $T_{\text {kin }}$ and density $n$. The plot shows that the ratio is mostly dependent on temperature and independent of the density of the gas. Right: $R_{404}$ as a function of kinetic temperature $T_{\text {kin }}$ and density $n$. The plot shows the strong dependence of the ratio on both temperature and density for densities $<10^{7} \mathrm{~cm}^{-3}$. The red plus signs indicate locations where the observed line ratios $R_{321}$ and $R_{404}$ are matched at the same time.

$15 \mathrm{~K} \mathrm{~km} \mathrm{~s}^{-1}$ (blue line in top panel of Fig. G.2). At this peak position the $4_{0,4}-3_{0,3}$ transition has an integrated intensity of $4 \mathrm{~K} \mathrm{~km} \mathrm{~s}^{-1}$ (blue line in bottom panel of Fig. G.2), while we find $R_{321}=0.25$ and $R_{422}=0.35$ (red line in top and bottom panel, respectively, of Fig. G.2). Further, the analysis of dust emission maps (Kauffmann et al. 2016) indicates $\mathrm{H}_{2}$ densities below $10^{5} \mathrm{~cm}^{-3}$. This limits the parameter space explored in our radiative transfer experiments. In our modeling, we thus require that the model intensities match the observed ones for $\mathrm{H}_{2}$ densities below $10^{5} \mathrm{~cm}^{-3}$. For these constraints we find that the data for the transitions near $218 \mathrm{GHz}$ can only be matched if we increase the column density to a multiple of the fiducial value (Fig. G.2, right panel, for an increased column density of $5 \times 10^{14} \mathrm{~cm}^{-2}$ ). The optical depth of the $3_{0,3}-2_{0,2}$ line is larger than one under these conditions. One might think that the high optical depth leads to massive biases in temperature estimates. This is, however, not the case: given a fixed line ratio, conditions with high optical depth can imply temperatures lower by $30 \%$ for optical depths $\gtrsim 5$ (i.e. our procedure, which assumes low optical depth, overestimates gas temperatures in regions of high optical depth).

\subsection{Observational uncertainties}

Further uncertainties in the temperature result from observational uncertainties in line ratios due to noise. To handle these uncertainties, we first obtain two additional temperature estimates per map position. These two estimates are calculated for line ratios that are equal to the observed value plus or minus the observed uncertainty in the line ratio. Conceptually these values bracket the range of possible temperatures for a given map position. The difference between these values represents the change in kinetic gas temperatures when changing the line ratio by twice its uncertainty (i.e. from $R_{i}-\sigma\left[R_{i}\right]$ to $R_{i}+\sigma\left[R_{i}\right]$, where $R_{i}$ and $\sigma\left[R_{i}\right]$ are the line ratio and its uncertainty). Half of this difference can thus be considered to represent the average temperature uncertainty associated with a line ratio uncertainty $\pm \sigma\left(R_{i}\right)$. In our analysis, we thus calculate for every map position the temperatures corresponding to $R_{i} \pm \sigma\left[R_{i}\right]$, and we report half of the difference between these temperatures as the observational uncertainty in kinetic gas temperatures. We are aware that the temperature uncertainties are asymmetric around the temperature value. However, we decided that the chosen method is the best way to present the uncertainties in this paper.

For some locations, the observations only yield upper limits to the line ratios. In such cases we take this upper limit to $R_{i}$, we calculate the associated temperature, and we report this resulting value as an upper limit to the kinetic gas temperature.

\section{Discussion}

The temperature maps of all velocity components of all sources are shown in Figs. H.1-H.7. Upper limits in the temperature maps are marked again with Xs. The corresponding pixels in the uncertainty maps are blanked in white. The measured temperatures range between 28 and $242 \mathrm{~K}$ at $218 \mathrm{GHz}$ and between 37 and $252 \mathrm{~K}$ at $291 \mathrm{GHz}$ (excluding upper limit values of the temperature). This shows that the gas temperatures are everywhere in the clouds much higher than the dust temperatures that are measured towards these clouds $(\sim 25 \mathrm{~K}$, Molinari et al. 2011). However, temperatures above $\sim 150 \mathrm{~K}$ have to be considered as lower limits since the radiative transfer models start to diverge significantly at these temperatures and the para$\mathrm{H}_{2} \mathrm{CO}$ line ratios are intrinsically insensitive to higher temperatures (Mangum \& Wootten 1993; Ginsburg et al. 2016).

We present the different plots we generated (integrated intensity maps, line ratio and uncertainty maps, temperature and uncertainty maps) for the $8-14 \mathrm{~km} \mathrm{~s}^{-1}$ component of the $20 \mathrm{~km} \mathrm{~s}^{-1}$ cloud in Fig. 4. In the last panel, we show a three-color image of Spitzer/GLIMPSE data (Churchwell et al. 2009, blue $=3.6 \mu \mathrm{m}$, green $=4.5 \mu \mathrm{m}$, red $=8.0 \mu \mathrm{m})$ of this source.

\subsection{Average temperatures}

To estimate and compare the overall temperatures of the clouds, we determined the weighted average of the temperature in each cloud for each velocity component, first, derived from the $218 \mathrm{GHz}$ lines and, second, from the $291 \mathrm{GHz}$ ladder. The squared reciprocal of the temperature uncertainties served as the weights. In this calculation, temperature upper limits were not included. The results are listed in Table 3 . In addition, we give the minimal and maximal temperature of each map in the table.

In most cases, the average temperature derived from the $291 \mathrm{GHz}$ data is higher than the average temperature derived from the $218 \mathrm{GHz}$ lines but the values agree within the 
$\mathrm{H}_{2} \mathrm{CO}$ integrated intensity maps (from left to right: $\mathrm{H}_{2} \mathrm{CO}\left(3_{0,3}-2_{0,2}\right), \mathrm{H}_{2} \mathrm{CO}\left(3_{2,1}-2_{2,0}\right), \mathrm{H}_{2} \mathrm{CO}\left(4_{0,4}-3_{0,3}\right)$, and $\mathrm{H}_{2} \mathrm{CO}\left(4_{2,2}-3_{2,1}\right)$ )
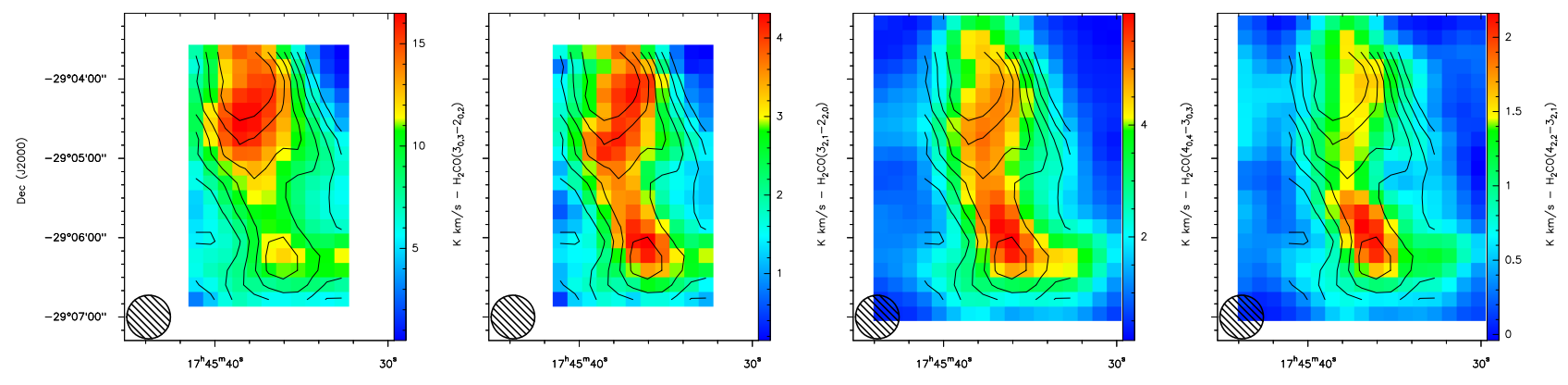

$\mathrm{H}_{2} \mathrm{CO}$ ratio (upper panels) and uncertainty maps (lower panels) (from left to right: $R_{321}, R_{422}$, and $R_{404}$ ).
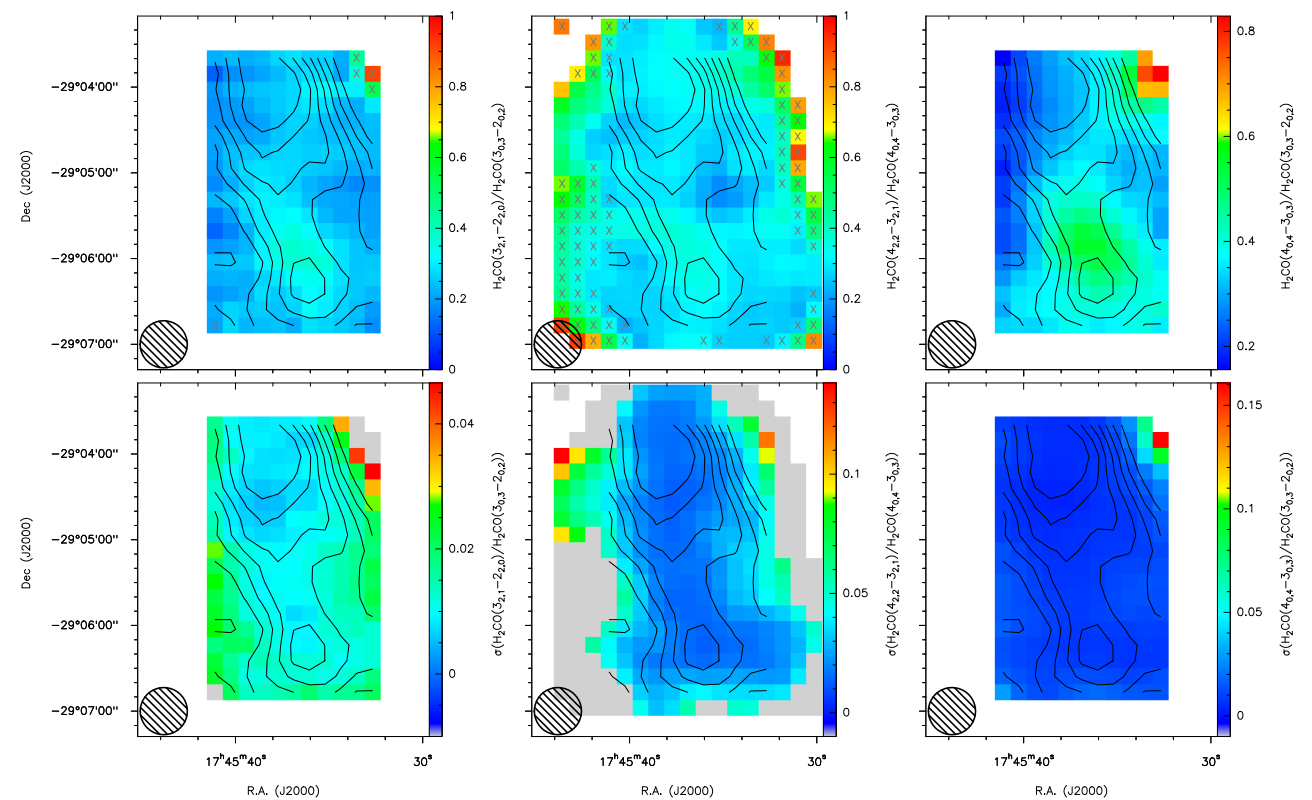

$\mathrm{H}_{2} \mathrm{CO}$ temperature (upper panels) and uncertainty maps (lower panels), derived from the $218 \mathrm{GHz}$ (left panels) and $291 \mathrm{GHz}$ data (right panels)
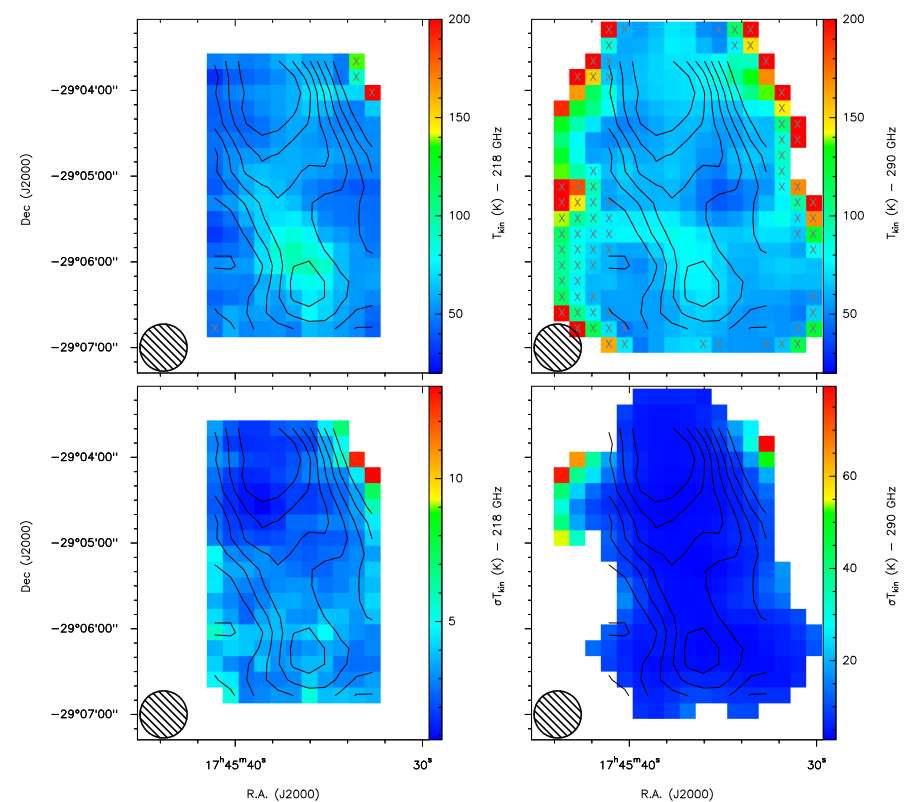

Spitzer/GLIMPSE RGB image (blue $=$ $3.6 \mu \mathrm{m}$, green $=4.5 \mu \mathrm{m}$, and red $=8.0 \mu \mathrm{m})$

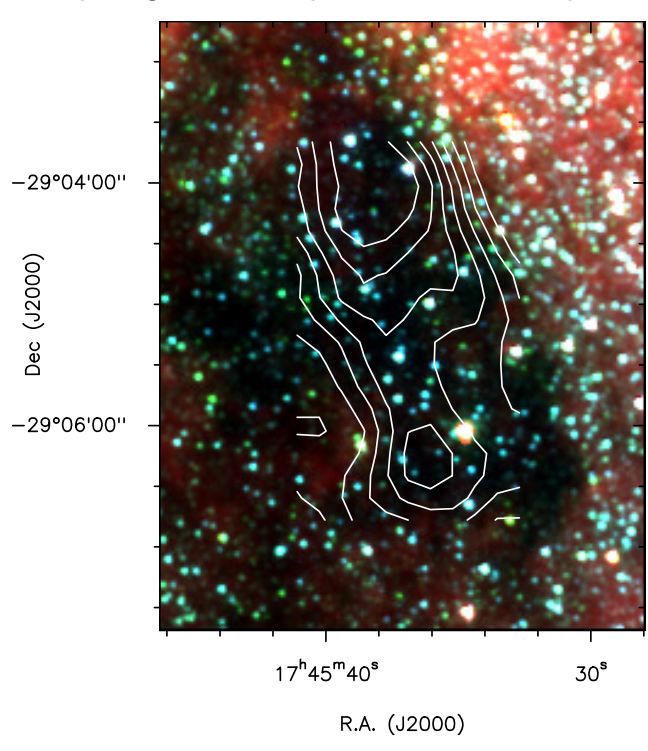

Fig. 4. Summary of the produced maps for the $8-14 \mathrm{~km} \mathrm{~s}^{-1}$ component of the $20 \mathrm{~km} \mathrm{~s}^{-1}$ cloud. The contours show the moment 0 map of the $\mathrm{H}_{2} \mathrm{CO}\left(3_{0,3}-2_{0,2}\right)$ transition, produced over the whole velocity range of the source (levels: $30-90 \%$ of the maximum in steps of $\left.10 \%\right)$. The circle in the lower left corner shows the 33" beam. 
Table 3. Minimal $T_{\mathrm{Min}}$, maximal $T_{\mathrm{Max}}$, and weighted average $T_{\text {Average }}$ temperatures, as well as $1 \sigma$ uncertainties of the weighted average temperature for all temperature maps (i.e. each source, each velocity component, and each frequency).

\begin{tabular}{|c|c|c|c|c|c|c|c|c|c|c|}
\hline \multirow[t]{2}{*}{ Source } & \multirow{2}{*}{$\begin{array}{l}\text { Velocity component } \\
\qquad\left(\mathrm{km} \mathrm{s}^{-1}\right)\end{array}$} & \multicolumn{4}{|c|}{$218 \mathrm{GHz}$} & \multicolumn{4}{|c|}{$291 \mathrm{GHz}$} & \multirow{2}{*}{$\begin{array}{c}T_{\text {Ginsburg }} \\
(\mathrm{K})\end{array}$} \\
\hline & & $\begin{array}{l}T_{\mathrm{Min}} \\
(\mathrm{K})\end{array}$ & $\begin{array}{c}T_{\text {Average }} \\
(\mathrm{K})\end{array}$ & $\begin{array}{c}\sigma_{\text {Average }} \\
(\mathrm{K})\end{array}$ & $\begin{array}{l}T_{\operatorname{Max}} \\
(\mathrm{K})\end{array}$ & $\begin{array}{l}T_{\mathrm{Min}} \\
(\mathrm{K})\end{array}$ & $\begin{array}{c}T_{\text {Average }} \\
(\mathrm{K})\end{array}$ & $\begin{array}{c}\sigma_{\text {Average }} \\
(\mathrm{K})\end{array}$ & $\begin{array}{l}T_{\operatorname{Max}} \\
(\mathrm{K})\end{array}$ & \\
\hline \multirow{5}{*}{$20 \mathrm{~km} \mathrm{~s}^{-1}$ cloud } & $-15-36$ & 28 & 58 & 16 & 133 & 37 & 62 & 11 & 163 & \multirow{5}{*}{64} \\
\hline & $0-6$ & 36 & 70 & 16 & 183 & 43 & 71 & 9 & 115 & \\
\hline & $8-14$ & 31 & 54 & 10 & 96 & 40 & 62 & 9 & 191 & \\
\hline & $27-33$ & 31 & 48 & 11 & 93 & 45 & 62 & 13 & 199 & \\
\hline & $16-80$ & 30 & 82 & 29 & 242 & 41 & 82 & 14 & 223 & \\
\hline \multirow{4}{*}{$50 \mathrm{~km} \mathrm{~s}^{-1}$ cloud } & $41-47$ & 44 & 87 & 18 & 142 & 53 & 91 & 15 & 150 & \multirow{2}{*}{91} \\
\hline & $57-63$ & 38 & 56 & 12 & 115 & 71 & 109 & 21 & 252 & \\
\hline & $-6-54$ & 29 & 50 & 14 & 203 & 41 & 61 & 12 & 212 & \multirow{5}{*}{82} \\
\hline & $-3-3$ & 61 & 81 & 16 & 180 & 56 & 77 & 17 & 140 & \\
\hline \multirow[t]{3}{*}{$\mathrm{G} 0.253+0.016$} & $16-22$ & 38 & 49 & 11 & 222 & 76 & 90 & 14 & 111 & \\
\hline & $36-42$ & 42 & 72 & 21 & 189 & 47 & 69 & 16 & 210 & \\
\hline & $75-81$ & 72 & 72 & & 72 & 95 & 109 & 25 & 183 & \\
\hline \multirow{2}{*}{ G0.411+0.050 } & $10-30$ & 41 & 49 & 8 & 132 & & & & & \multirow{2}{*}{57} \\
\hline & $19-25$ & 49 & 59 & 11 & 197 & & & & & \\
\hline \multirow{2}{*}{ G0.480-0.006 } & $19-44$ & 32 & 40 & 8 & 117 & 50 & 69 & 18 & 239 & \multirow{2}{*}{84} \\
\hline & $27-33$ & 42 & 56 & 12 & 161 & 65 & 84 & 14 & 189 & \\
\hline \multirow{2}{*}{ Sgr C } & $-60--46$ & & & & & 54 & 72 & 8 & 173 & \multirow{2}{*}{53} \\
\hline & $-55--49$ & & & & & 43 & 59 & 7 & 191 & \\
\hline \multirow{2}{*}{ Sgr D } & $-17--14$ & & & & & 47 & 56 & 8 & 80 & \multirow{2}{*}{52} \\
\hline & $-19--13$ & & & & & 39 & 50 & 7 & 66 & \\
\hline
\end{tabular}

Notes. The last column gives the average temperature of each source from the survey of Ginsburg et al. (2016).

$1 \sigma$ temperature uncertainty. In two cases, however, the temperature difference is rather large $\left(50 \mathrm{~km} \mathrm{~s}^{-1}\right.$ cloud: $57-63 \mathrm{~km} \mathrm{~s}^{-1}$ component, G0.253+0.016: 16-22 $\mathrm{km} \mathrm{s}^{-1}$ component). In Fig. H.8, we visualize these results in temperature box plots.

To check the validity of our results, we compared our temperature measurements to the $\mathrm{H}_{2} \mathrm{CO} 218 \mathrm{GHz}$ survey of Ginsburg et al. (2016). For each source, we averaged their temperature map (their Fig. 7c) over the sizes of our $218 \mathrm{GHz}$ OTF maps (the $291 \mathrm{GHz}$ OTF maps for Sgr C and Sgr D). The obtained values are shown in the last column of Table 3 . These average temperatures are roughly consistent with our results, except for G0.480-0.006. Here, the average temperature of Ginsburg et al. (2016) is much higher than our values at $218 \mathrm{GHz}$. However, our $291 \mathrm{GHz}$ measurements do show a high temperature component. We conclude that, on average, our study yields similar temperatures for the observed clouds as the survey of Ginsburg et al. (2016).

Comparing the average temperature of the whole velocity range of each source, we noticed a large spread of temperatures between $40 \mathrm{~K}$ in G0.480-0.006 and $82 \mathrm{~K}$ in the $50 \mathrm{~km} \mathrm{~s}^{-1}$ cloud. There is no clear correlation of the average temperature with the location of the clouds in the CMZ.

\subsection{Temperature gradients within clouds}

Table 3 shows that the average temperatures of the different velocity components in the sources are not the same. Differences of $\sim 30 \mathrm{~K}$ are observed. This indicates the presence of temperature gradients in the sources. These results show that averaging the temperatures over the whole velocity range of the clouds can yield misleading values of the temperature. It is thus important to look at the temperatures of the gas with different velocities to understand the underlying temperature structures of the clouds.
The $20 \mathrm{~km} \mathrm{~s}^{-1}$ cloud and G0.253+0.016 even show temperature gradients across the sources within one velocity component. In Fig. 4, the temperature map of the $8-14 \mathrm{~km} \mathrm{~s}^{-1}$ component of the $20 \mathrm{~km} \mathrm{~s}^{-1}$ cloud is plotted. There is a clear temperature gradient from $\sim 60-70 \mathrm{~K}$ at the north-east side of the cloud to $\sim 110-120 \mathrm{~K}$ in the southern part. A similar gradient is seen in the 36-42 $\mathrm{km} \mathrm{s}^{-1}$ component of G0.253+0.016 (Fig. H.3, fourth panel), with the temperature increasing from $\sim 70 \mathrm{~K}$ in the north of the cloud to more than $150 \mathrm{~K}$ in the southern part.

In a forthcoming paper, we will compare the temperature structures of the clouds with tracers of shocks (e.g. observations of $\mathrm{SiO}$, Class I methanol masers) or star formation (Class II methanol masers, water masers) to apprehend the cause for the different temperatures.

\subsection{Evidence for heating by turbulence}

To better understand the temperature structures in our clouds, we investigate the energy balance in the gas following the analysis of Ginsburg et al. (2016). We do not consider stellar heating or energy injection through supernovae since these processes can only explain local heating of the clouds, but not their overall high gas temperature. Ao et al. (2013) already excluded diffuse $\mathrm{X}$-rays as the main heating source, leaving only cosmic ray and mechanical heating (turbulence) as the energetically important heating processes. Ginsburg et al. (2016) concluded that cosmic ray heating is either irrelevant in the $\mathrm{CMZ}$ or the heating is nonuniform since uniform heating cannot simultaneously explain the high $(>100 \mathrm{~K})$ and low $(<50 \mathrm{~K})$ temperatures measured in the CMZ. The detected temperature gradients in our clouds also exclude the latter, except if the cosmic ray heating is non-uniform on very small scales $(\sim 1 \mathrm{pc})$. We will produce similar gas temperature vs. line width plots (as their Fig. 9) for the 218 and $291 \mathrm{GHz}$ temperatures and compare our data to the same models 

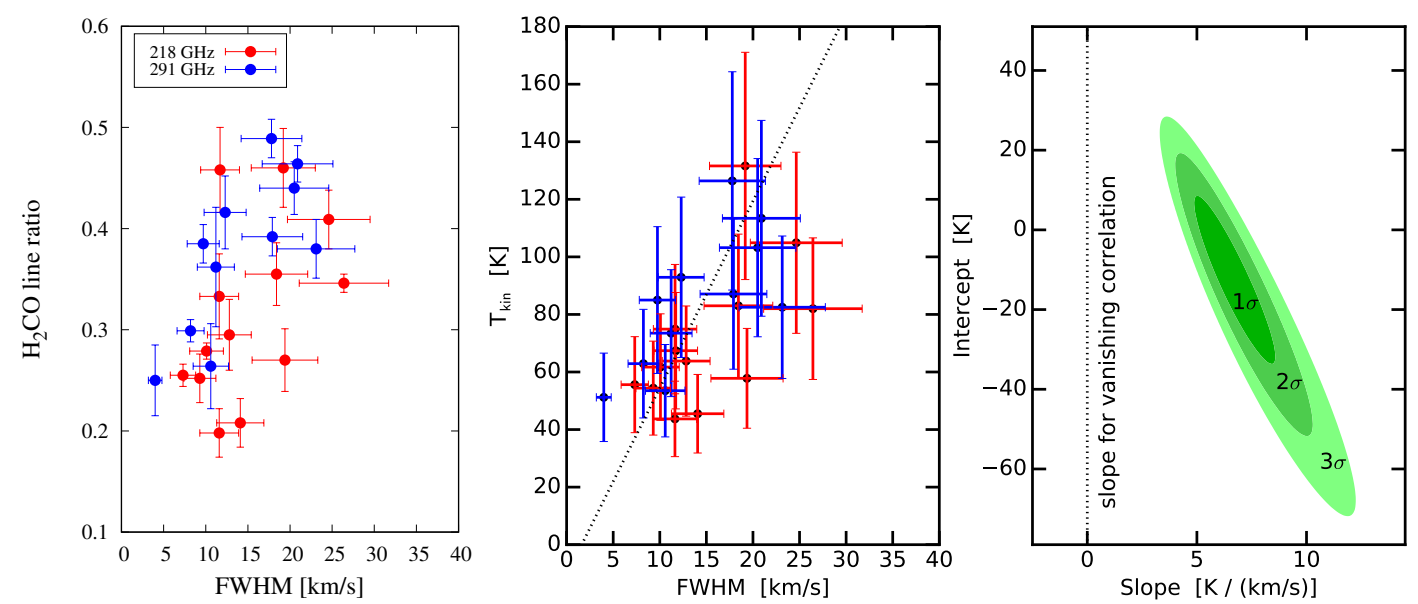

Fig. 5. Left and middle panels: correlation plots of $\mathrm{H}_{2} \mathrm{CO}$ integrated intensity ratio and kinetic temperature, respectively vs. line width. The error bars present the standard deviation of the weighted average for the integrated intensity ratio, $20 \%$ uncertainty in the line width and $30 \%$ uncertainty in the temperature. The best linear fit to the temperature data using the total least-square method (Vanderplas et al. 2012) is shown as the solid line. Right panel: The possible slope and intercept values with the 1,2, and $3 \sigma$ likelihood contours.

as used in Ginsburg et al. (2016) to narrow down the parameter space of possible heating models.

To collect the data of these plots, we chose one or several positions in the 218 and $291 \mathrm{GHz}$ temperature maps of the $6 \mathrm{~km} \mathrm{~s}^{-1}$ broad velocity components of each source where none of the pixels in a $33^{\prime \prime} \times 33^{\prime \prime}$ tile (roughly corresponding to the size of the beam) around these positions are upper limits. The boxes are overlayed on the temperature maps in Figs. H.1-H.7. We then determined the weighted average temperature within these boxes, using the squared reciprocal of the temperature uncertainties as weights. Averaging the $\mathrm{H}_{2} \mathrm{CO}\left(3_{0,3}-2_{0,2}\right)$ spectra of the nine pixels in the boxes, we fitted the line with one or more velocity components depending on the source. We then assigned each average temperature value the line width of the corresponding velocity component. We excluded the $20 \mathrm{~km} \mathrm{~s}^{-1}$ cloud from this analysis because we could not fit the line width of the different components unambiguously. To make sure that any trend we see is real and not due to the radiative transfer modeling, we also obtained the average line ratios $R_{321}$ and $R_{422}$ in those tiles. The values for line ratio, temperature, and line width are listed in Table I.1. In Fig. 5, we show the relation of line ratio (left panel) and temperature (middle panel), respectively, versus line width.

Considering the statistical uncertainties associated with both parameters ( $20 \%$ for the line width, $30 \%$ for the temperature), we adopt the method of total least squares (Vanderplas et al. 2012) to measure the slope of the $\mathrm{d} v-T_{\text {kin }}$ relation. Since we are integrating the emission of the $\mathrm{H}_{2} \mathrm{CO}$ lines over a fixed velocity range, it is safe to assume that the temperature and line width measurements are uncorrelated, so we set the covariance between them to be zero. Figure 5 shows a statistically significant positive correlation, which suggests that regions of higher line widths in our clouds are expected to have higher gas temperature. This implies that turbulence might play a direct role in gas heating.

Figure 6 shows a collection of thermal equilibrium models computed using the DESPOTIC (Krumholz 2014) code. Each model uses the heating terms specified in the legend and both line and dust cooling. The models compute the equilibrium temperature achieved at the specified density. The primary variable being varied is the line width, which is treated as an observational proxy for the $3 \mathrm{D}$ velocity dispersion following the equation $\sigma_{3 \mathrm{D}}=\sqrt{3} \sigma_{1 \mathrm{D}}$. In contrast with Ginsburg et al. (2016), who observed a wider range in densities and environments, our data show a strong trend between the observed temperature and the velocity dispersion. These are readily explained by the $n=10^{5} \mathrm{~cm}^{-3}$ model that assumes that each cloud has a line-of-sight length of $1 \mathrm{pc}$. These observations therefore significantly strengthen the case put forth by Ao et al. (2013) and Ginsburg et al. (2016) that the densest Galactic center clouds are predominantly heated by turbulence.

\subsection{Density constraints}

Following Mangum \& Wootten (1993), the integrated intensity ratio of $\mathrm{H}_{2} \mathrm{CO} \Delta J=1$ lines with different $\mathrm{J}$, but the same $K_{\mathrm{a}}$ quantum numbers constrains the density of the gas. Figure 3 (right panel) shows that the $R_{404}$ ratio depends strongly on both temperature and density for densities $<10^{7} \mathrm{~cm}^{-3}$, while the $R_{321}$ ratio only depends on temperature (Fig. 3, left panel). Thus, combining the measurements of the $R_{321}$ and $R_{422}$ ratios that yield the temperature with the $R_{404}$ ratio, we can estimate the density in the clouds. The red plus signs in Fig. 3 show the locations where the $R_{321}$ and $R_{404}$ ratios are matched at the same time. Due to the significant dependence of the density estimate on the temperature estimate and our temperature uncertainties of $\sim 30 \%$, we cannot compute density maps of our clouds. However, we constrain the density of the widespread warm gas in our clouds to $10^{4}-10^{6} \mathrm{~cm}^{-3}$. Efforts are ongoing to further constrain this range using the $\mathrm{cm}$ transitions of $\mathrm{H}_{2} \mathrm{CO}$ (Ginsburg et al., in prep.).

\section{Conclusion}

In this paper, we present $\mathrm{H}_{2} \mathrm{CO}$ observations of five and seven CMZ clouds at 218 and $291 \mathrm{GHz}$, respectively. Combining integrated intensity $\mathrm{H}_{2} \mathrm{CO}$ line ratios with radiative transfer models, we obtain gas temperature maps for our clouds. The two different sets of $\mathrm{H}_{2} \mathrm{CO}$ lines $\left(\mathrm{H}_{2} \mathrm{CO}(3-2)\right.$ at $218 \mathrm{GHz}$ and $\mathrm{H}_{2} \mathrm{CO}(4-3)$ at $\left.291 \mathrm{GHz}\right)$ yield two independent estimates of the gas temperature.

Our observations at $218 \mathrm{GHz}$ are a factor of $\sim 1.5$ deeper than previous $\mathrm{H}_{2} \mathrm{CO} \mathrm{CMZ}$ observations by Ginsburg et al. (2016, 


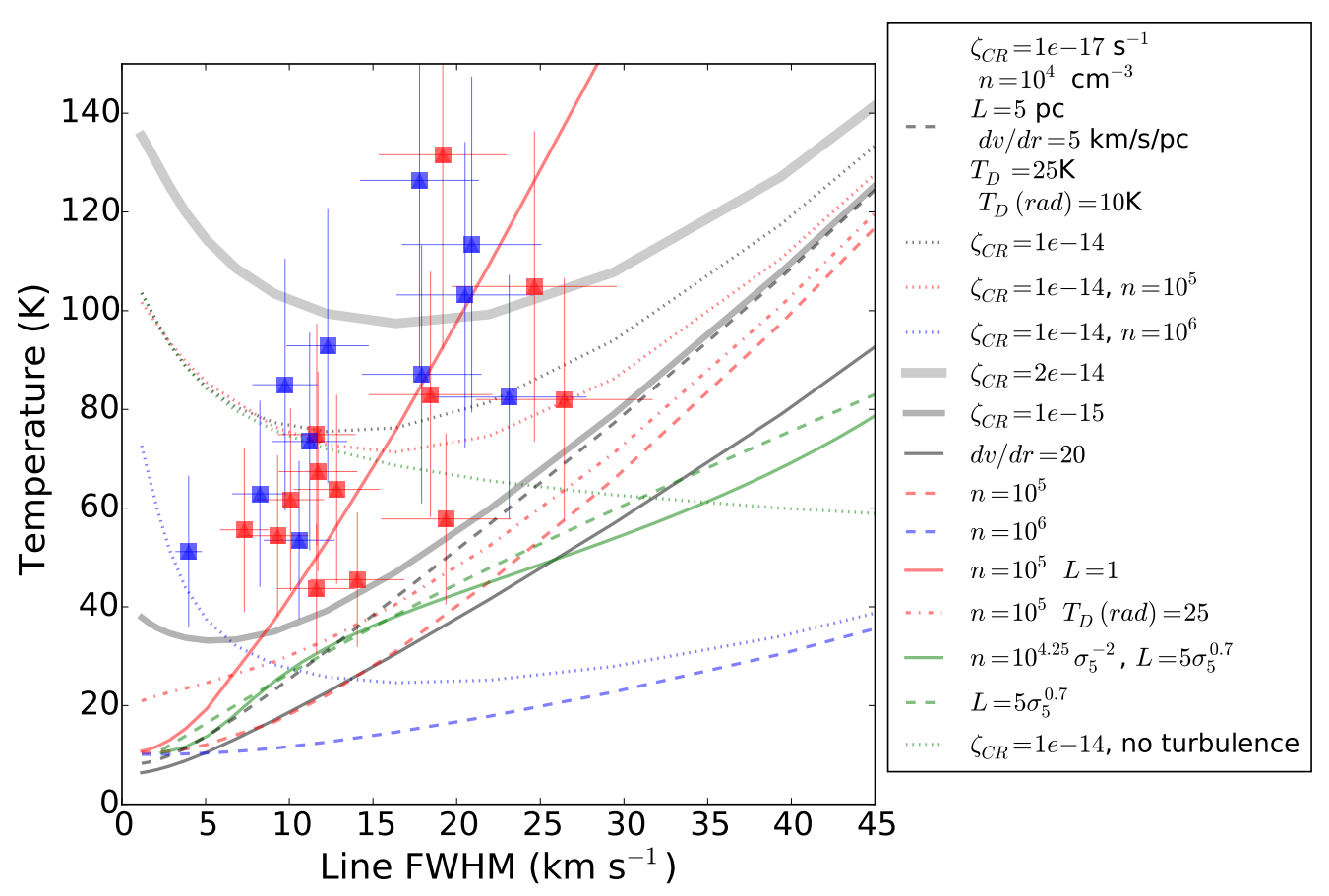

Fig. 6. Temperature versus line width plot, overlayed with thermal equilibrium models computed using the DESPOTIC (Krumholz 2014) code (see also Fig. 9 of Ginsburg et al. 2016). The data is consistent with the $n=10^{5} \mathrm{~cm}^{-3}$ model.

compare our median rms values of $\sim 45 \mathrm{mK}$ per pixel to their noise level of $70 \mathrm{mK}$ per pixel). While Ginsburg et al. focus on the global temperature differences in the CMZ, we disentangle the different velocity components of the gas in our sources and investigate their temperature structures. This is a significant step since the CMZ clouds exhibit the widest velocity components observed in our galaxy.

From a comparison of the $\mathrm{H}_{2} \mathrm{CO}$ main lines at 218 and $291 \mathrm{GHz}$, we accessed that the $\mathrm{H}_{2} \mathrm{CO}\left(3_{0,3}-2_{0,2}\right)$ line is optically thick in some parts of the clouds. Combining the observed line ratios $R_{321}$ and $R_{404}$, we constrain the density of the warm cloud gas to $10^{4}-10^{6} \mathrm{~cm}^{-3}$.

Our temperature maps at 218 and $291 \mathrm{GHz}$ show clear temperature gradients in our clouds. This indicates that heating mechanisms that act on the bulk of the molecular gas cannot be the main heating sources. Cosmic ray heating is only possible if the heating is non-uniform on very small scales. In a following paper, we will compare our results with complementary observations of shock and star formation tracers, as well as supernova remnants in the clouds to understand if these gradients are caused by local heating through cloud collisions, feedback from new born stars, or the explosion of stars.

Comparing the line widths of the main $\mathrm{H}_{2} \mathrm{CO}$ lines at 218 and $291 \mathrm{GHz}$ with the measured temperatures at selected positions in our clouds, we found a clear positive correlation between these two parameters. This indicates that turbulence plays an important role in the heating of the gas. Our data is consistent with a turbulence model with a density $n=10^{5} \mathrm{~cm}^{-3}$ that assumes that each cloud has a line-of-sight length of $1 \mathrm{pc}$.

Acknowledgements. We thank the APEX team and MPIfR observers for carrying out these service-mode observations. The authors are thankful for the helpful comments of the anonymous referee. T.P. acknowledges support from the Deutsche Forschungsgemeinschaft, DFG via the SPP (priority program) 1573 Physics of the ISM. This research has made use of NASA's Astrophysics Data System Bibliographic Services.

\section{References}

Ao, Y., Henkel, C., Menten, K. M., et al. 2013, A\&A, 550, A135 Blum, R. D., Damineli, A., \& Conti, P. S. 1999, AJ, 117, 1392

Churchwell, E., Babler, B. L., Meade, M. R., et al. 2009, PASP, 121, 213

Ginsburg, A., Henkel, C., Ao, Y., et al. 2016, A\&A, 586, A50

Güsten, R., Walmsley, C. M., \& Pauls, T. 1981, A\&A, 103, 197

Güsten, R., Nyman, L. A., Schilke, P., et al. 2006, A\&A, 454, L13

Heyminck, S., Kasemann, C., Güsten, R., de Lange, G., \& Graf, U. U. 2006, A\&A, 454, L21

Hüttemeister, S., Wilson, T. L., Bania, T. M., \& Martin-Pintado, J. 1993, A\&A, 280,255

Immer, K., Schuller, F., Omont, A., \& Menten, K. M. 2012, A\&A, 537, A121

Kauffmann, et al. 2016, submitted

Klein, B., Hochgürtel, S., Krämer, I., et al. 2012, A\&A, 542, L3

Klein, T., Ciechanowicz, M., Leinz, C., et al. 2014, IEEE Transactions on Terahertz Science and Technology, 4, 588

Kruijssen, J. M. D., \& Longmore, S. N. 2013, MNRAS, 435, 2598

Krumholz, M. R. 2014, MNRAS, 437, 1662

Lis, D. C., Li, Y., Dowell, C. D., \& Menten, K. M. 1999, in The Universe as Seen by ISO, eds. P. Cox, \& M. Kessler, ESA SP, 427, 627

Longmore, S. N., Bally, J., Testi, L., et al. 2013, MNRAS, 429, 987

Mangum, J. G., \& Wootten, A. 1993, ApJS, 89, 123

Mehringer, D. M., Goss, W. M., Lis, D. C., Palmer, P., \& Menten, K. M. 1998, ApJ, 493, 274

Mills, E. A. C., \& Morris, M. R. 2013, ApJ, 772, 105

Molinari, S., Bally, J., Noriega-Crespo, A., et al. 2011, ApJ, 735, L33

Morris, M., \& Serabyn, E. 1996, ARA\&A, 34, 645

Ott, J., Weiß, A., Staveley-Smith, L., Henkel, C., \& Meier, D. S. 2014, ApJ, 785, 55

Pety, J. 2005, in SF2A-2005: Semaine de 1'Astrophysique Française, eds. F. Casoli, T. Contini, J. M. Hameury, \& L. Pagani (Les Ulis: EDP Sciences), 721

Sawada, M., Tsujimoto, M., Koyama, K., et al. 2009, PASJ, 61, S209

Schöier, F. L., van der Tak, F. F. S., van Dishoeck, E. F., \& Black, J. H. 2005, A\&A, 432, 369

Schuller, F., Menten, K. M., Contreras, Y., et al. 2009, A\&A, 504, 415

Shirley, Y. L. 2015, PASP, 127, 299

van der Tak, F. F. S., Black, J. H., Schöier, F. L., Jansen, D. J., \& van Dishoeck, E. F. 2007, A\&A, 468, 627

Vanderplas, J., Connolly, A., Ivezić, Ž., \& Gray, A. 2012, in Proc. Conference on Intelligent Data Understanding (CIDU), 47

Vassilev, V., Meledin, D., Lapkin, I., et al. 2008, A\&A, 490, 1157

Wiesenfeld, L., \& Faure, A. 2013, MNRAS, 432, 2573

Yusef-Zadeh, F., Hewitt, J. W., Arendt, R. G., et al. 2009, ApJ, 702, 178 
Appendix A: Calibration

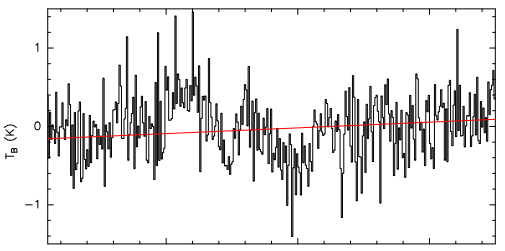

(a)

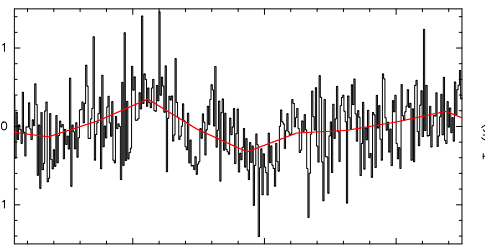

(b)

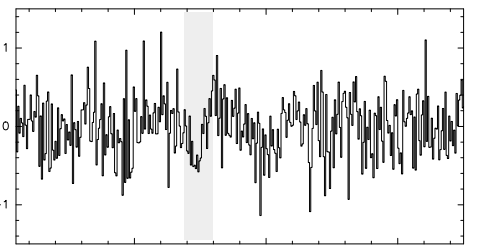

(c)

Fig. A.1. Example spectra of the $\mathrm{H}_{2} \mathrm{CO}\left(3_{0,3}-2_{0,2}\right)$ transition of target $\mathrm{G} 0.253+0.016$ to show the spline-fitting of bad baselines. Left: input spectrum showing a bad baseline. The red line shows a linear baseline for comparison. Middle: input spectrum with the spline spectrum (red) overlayed. Right: baseline-subtracted spectrum. The gray box shows a baseline feature that was not removed in the spline-fitting due to its narrow width.

\section{Appendix B: Spectra}

$20 \mathrm{~km} \mathrm{~s}^{-1}$ cloud
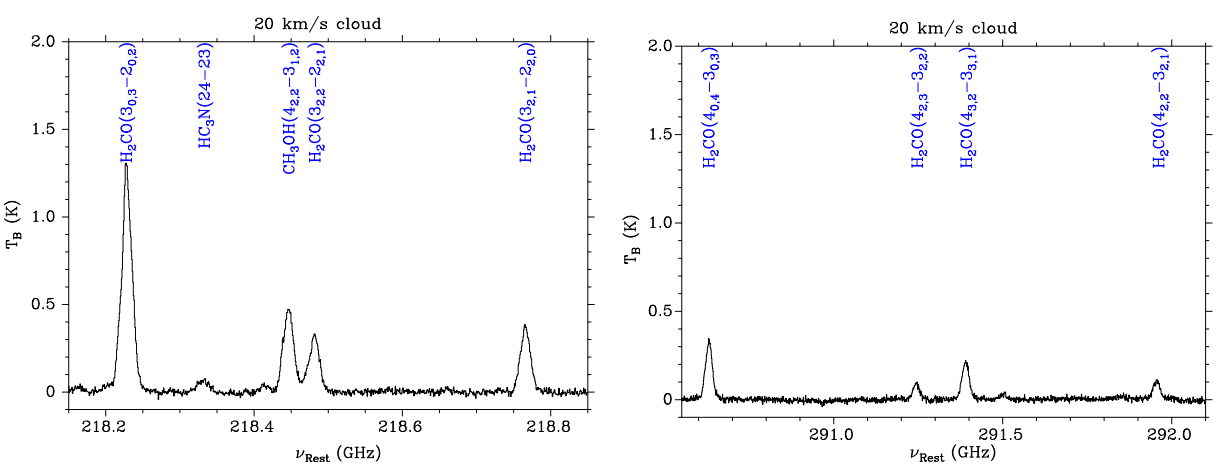

$50 \mathrm{~km} \mathrm{~s}^{-1}$ cloud
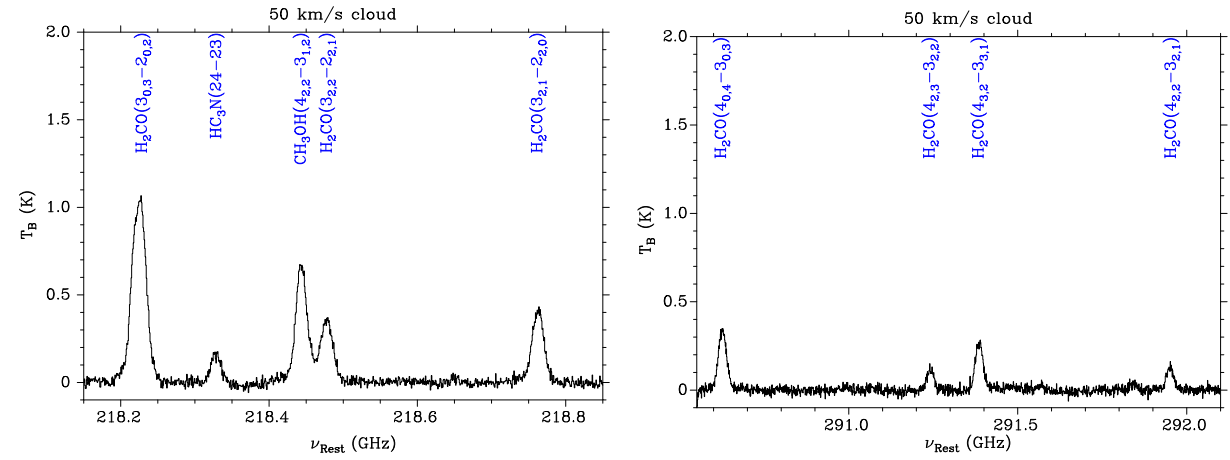

$\mathrm{G} 0.253+0.016$
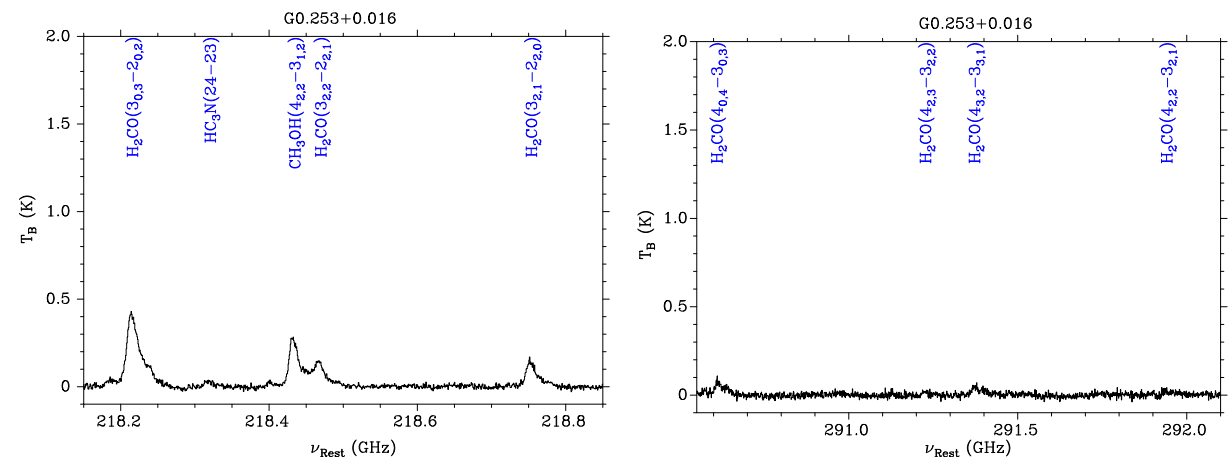

Fig. B.1. Spectra of para- $\mathrm{H}_{2} \mathrm{CO}$ transitions at 218 (left panels) and $291 \mathrm{GHz}$ (right panels), averaged over the whole OTF map of each source. $\mathrm{H}_{2} \mathrm{CO}$ as well as other lines are marked in the spectra. 
A\&A 595, A94 (2016)

\section{$\mathrm{G} 0.411+0.050$}
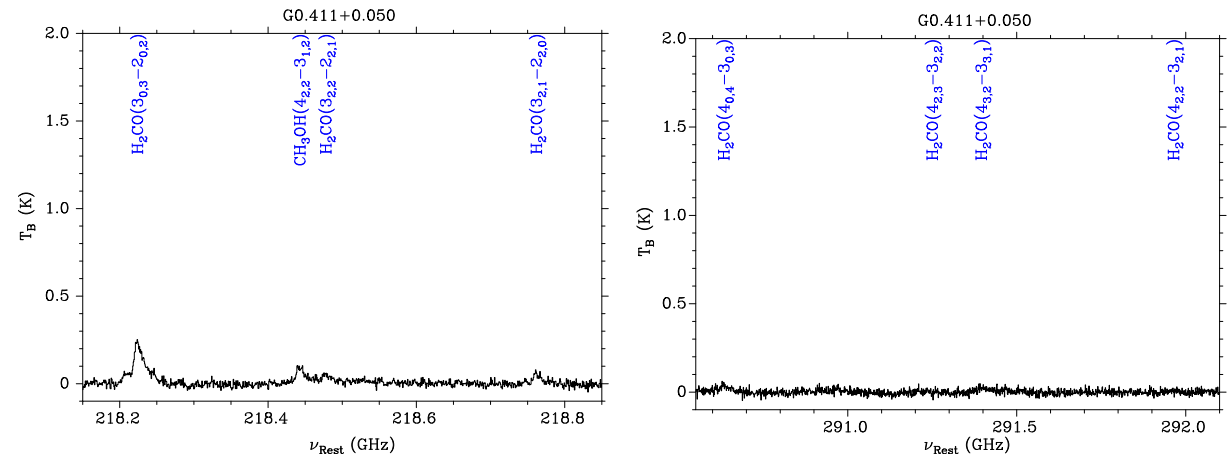

G0.480-0.006

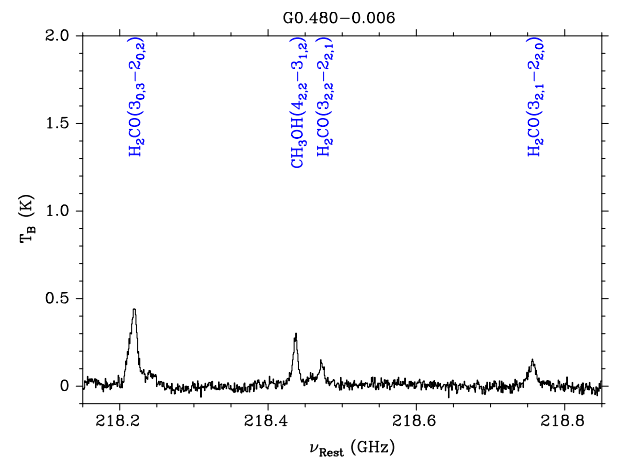

Sgr C
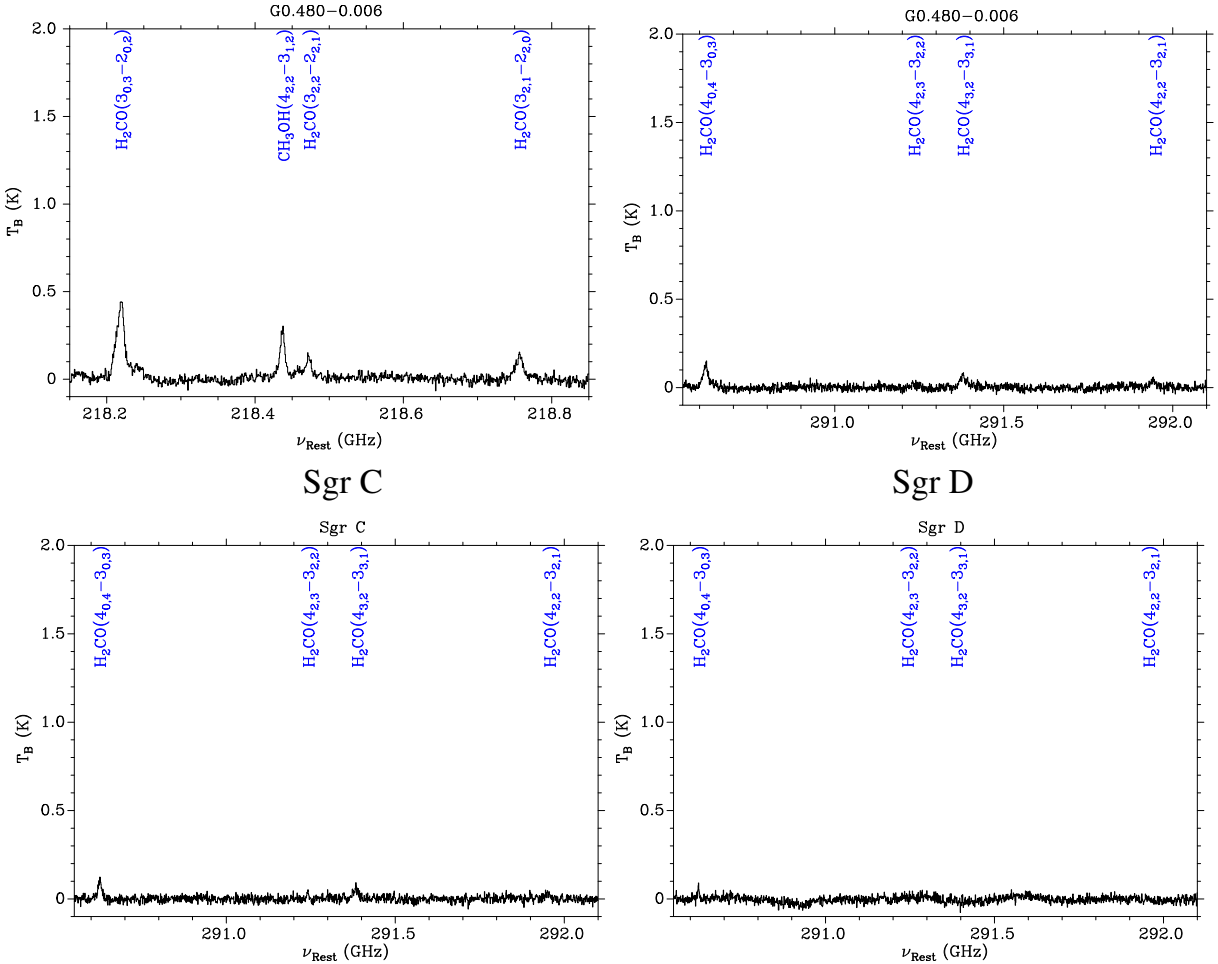

Sgr D

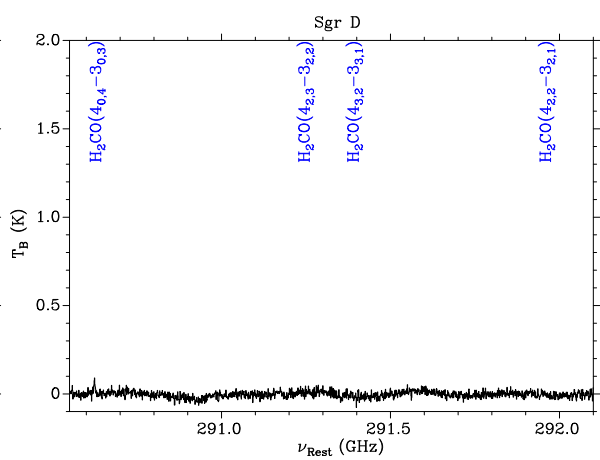

Fig. B.1. continued.
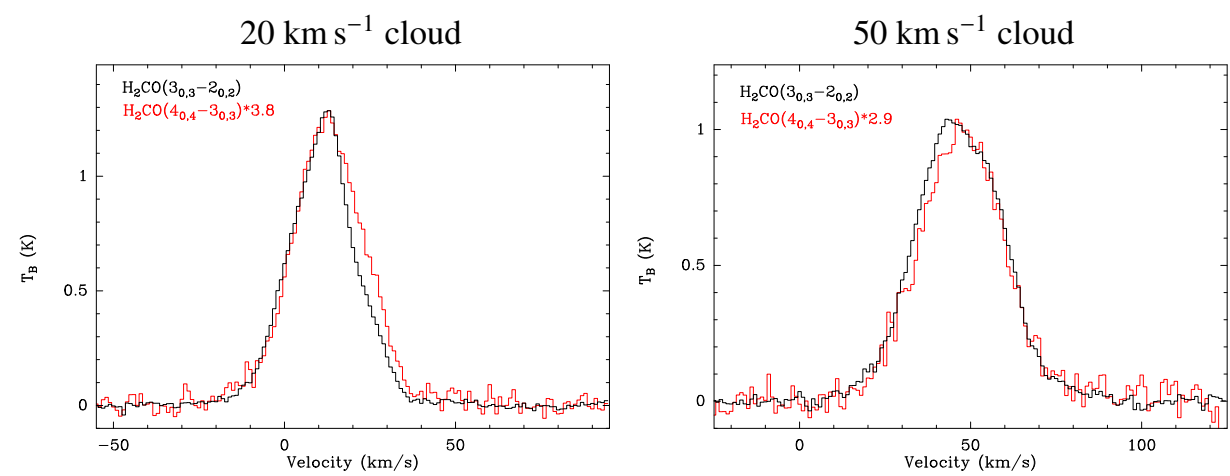

Fig. B.2. The spectra of the $\mathrm{H}_{2} \mathrm{CO}\left(3_{0,3}-2_{0,2}\right)$ and $\mathrm{H}_{2} \mathrm{CO}\left(4_{0,4}-3_{0,3}\right)$ transitions for the $20 \mathrm{~km} \mathrm{~s}^{-1}$ cloud (left) and the $50 \mathrm{~km} \mathrm{~s}^{-1}$ cloud (right) plotted on top of each other. The spectra of $\mathrm{H}_{2} \mathrm{CO}\left(4_{0,4}-3_{0,3}\right)$ were multiplied by a factor of 3.8 and 2.9 in the left and right panels, respectively, to bring the two lines to the same scale. The plots show that the two transitions have comparable width in both sources. 
K. Immer et al.: Temperature structures in Galactic center clouds

\section{Appendix C: Methanol contamination}

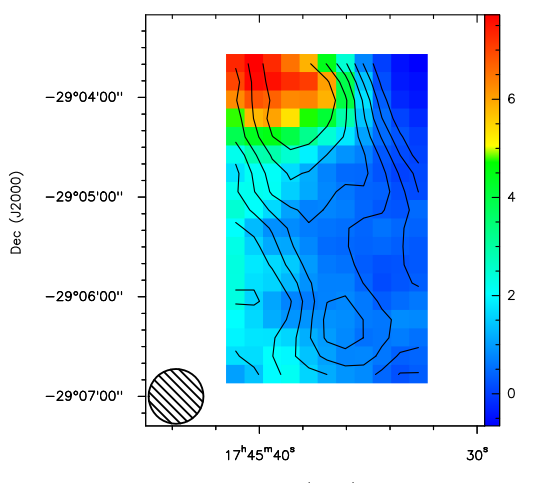

(a) $\mathrm{H}_{2} \mathrm{CO}\left(3_{0,3}-2_{0,2}\right)$

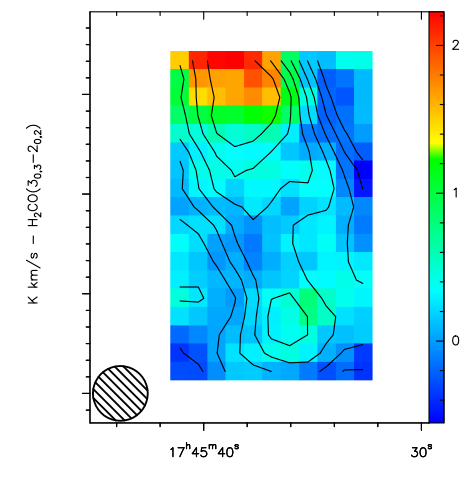

R.A. (J2000)

(b) $\mathrm{H}_{2} \mathrm{CO}\left(3_{2,1}-2_{2,0}\right)$

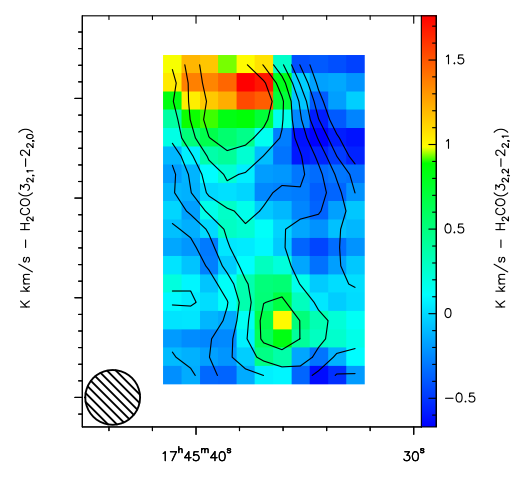

R.A. (J2000)

(c) $\mathrm{H}_{2} \mathrm{CO}\left(3_{2,2}-2_{2,1}\right)$

Fig. C.1. Comparison of the integrated emission of $\mathrm{H}_{2} \mathrm{CO}\left(3_{0,3}-2_{0,2}\right), \mathrm{H}_{2} \mathrm{CO}\left(3_{2,1}-2_{2,0}\right)$ and $\mathrm{H}_{2} \mathrm{CO}\left(3_{2,2}-2_{2,1}\right)$ of the $20 \mathrm{~km} \mathrm{~s}^{-1}$ cloud over the velocity range 27-33 $\mathrm{km} \mathrm{s}^{-1}$, showing the contamination of the $\mathrm{H}_{2} \mathrm{CO}\left(3_{2,2}-2_{2,1}\right)$ line with methanol emission at the south of the cloud. The contours show the moment 0 map of the $\mathrm{H}_{2} \mathrm{CO}\left(3_{0,3}-2_{0,2}\right)$ transition, produced over the whole velocity range of the source (levels: $30-90 \%$ of the maximum in steps of 10\%) The circle in the lower left corner shows the $33^{\prime \prime}$ beam. 


\section{Appendix D: $\mathrm{H}_{2} \mathrm{CO}\left(3_{0,3}-2_{0,2}\right)$ spectra}
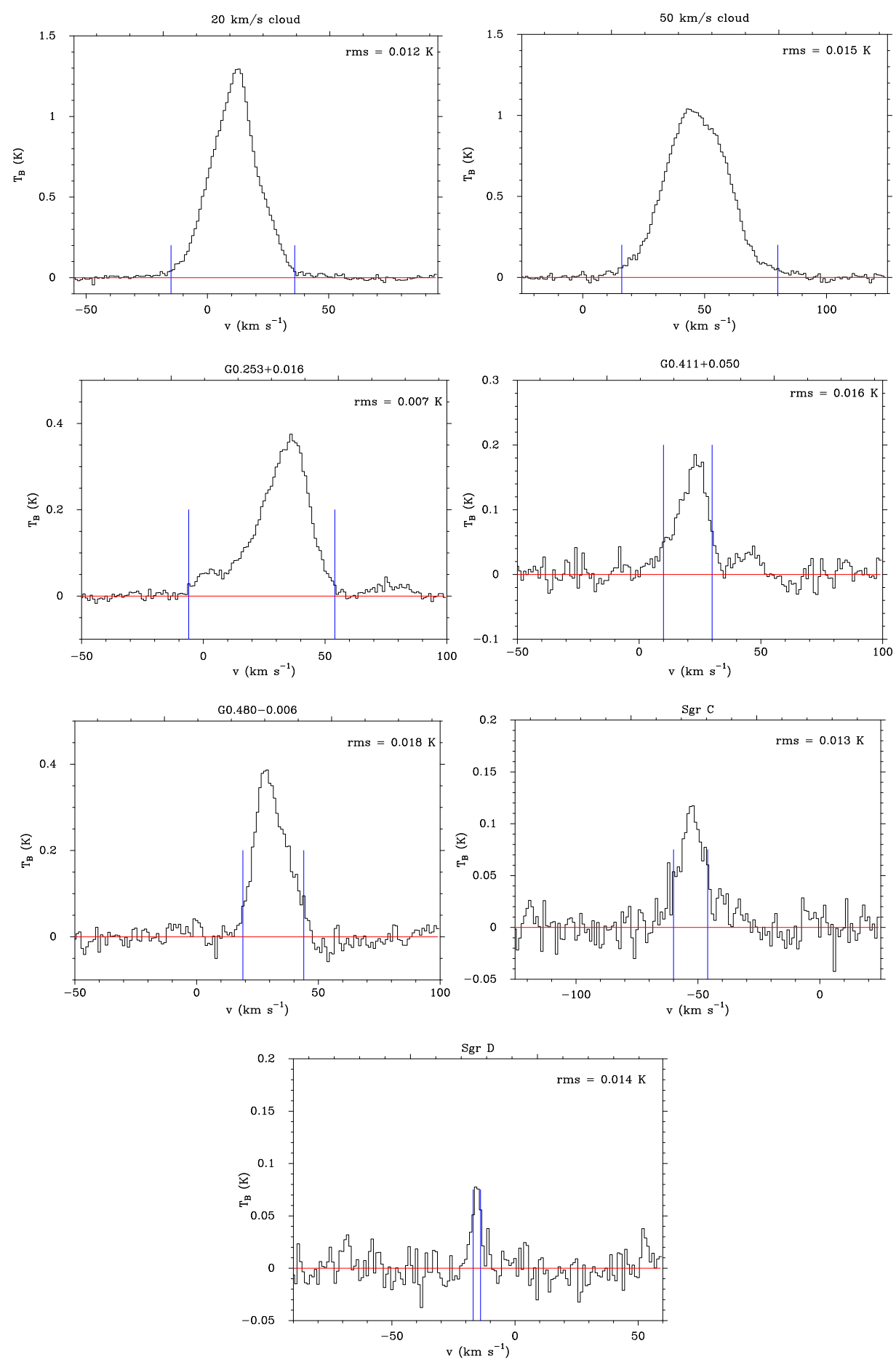

Fig. D.1. $\mathrm{H}_{2} \mathrm{CO}\left(3_{0,3}-2_{0,2}\right)$ spectra, averaged over the whole OTF map of each source. The blue lines indicate the velocity range of the whole source where the line emission is above $3 \sigma$. The red line shows the fitted baseline (rms value in upper right corner). 
K. Immer et al.: Temperature structures in Galactic center clouds

\section{Appendix E: Integrated intensity maps}
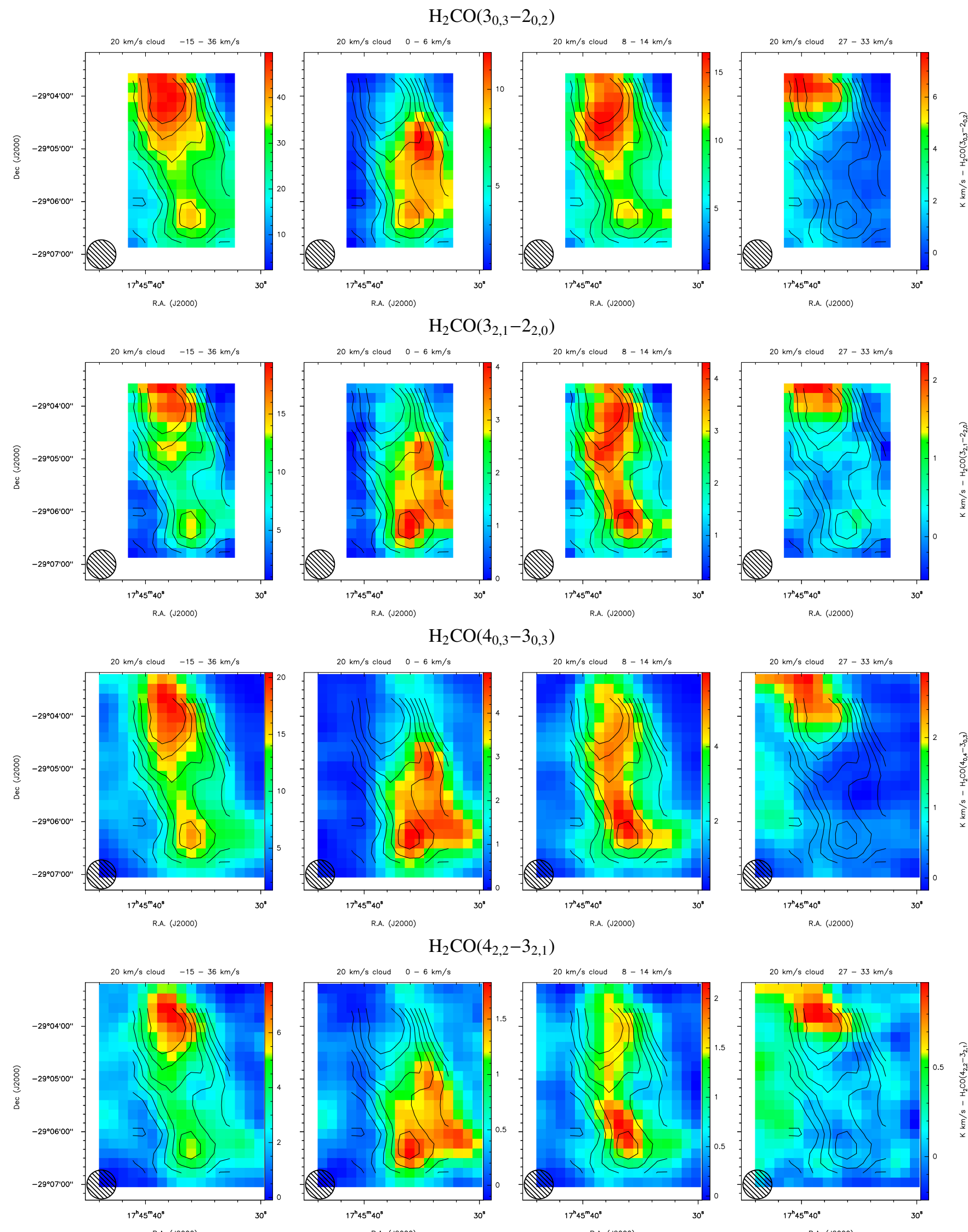

Fig. E.1. Integrated intensities of the para- $\mathrm{H}_{2} \mathrm{CO}$ lines at 218 and $291 \mathrm{GHz}$ in the $20 \mathrm{~km} \mathrm{~s}^{-1}$ cloud. The contours show the moment 0 map of the $\mathrm{H}_{2} \mathrm{CO}\left(3_{0,3}-2_{0,2}\right)$ transition, produced over the whole velocity range of the source (levels: $30-90 \%$ of the maximum in steps of $10 \%$ ). The circle in the lower left corner shows the $33^{\prime \prime}$ beam. 
A\&A 595, A94 (2016)

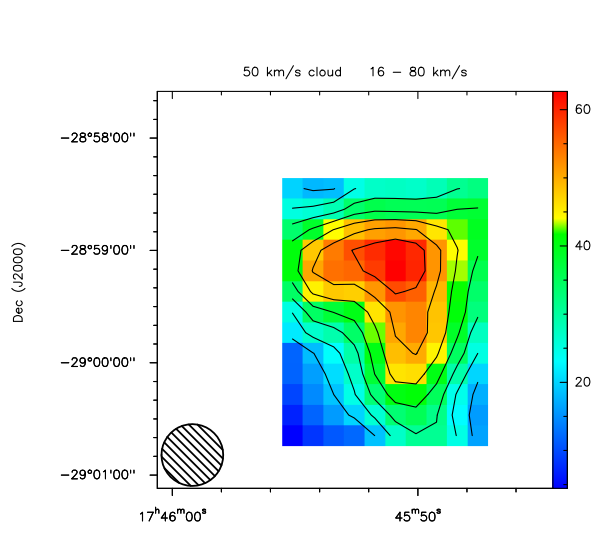

$\mathrm{H}_{2} \mathrm{CO}\left(3_{0,3}-2_{0,2}\right)$

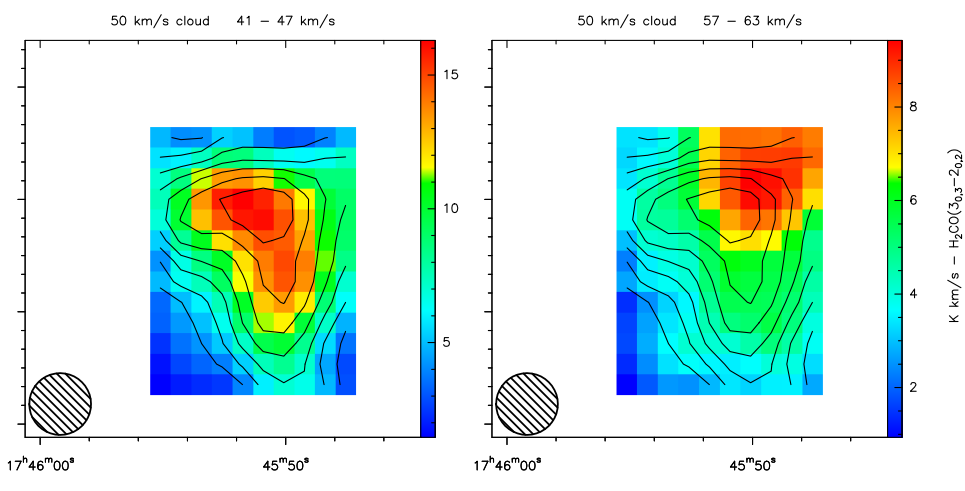

$\mathrm{H}_{2} \mathrm{CO}\left(3_{2,1}-2_{2,0}\right)$
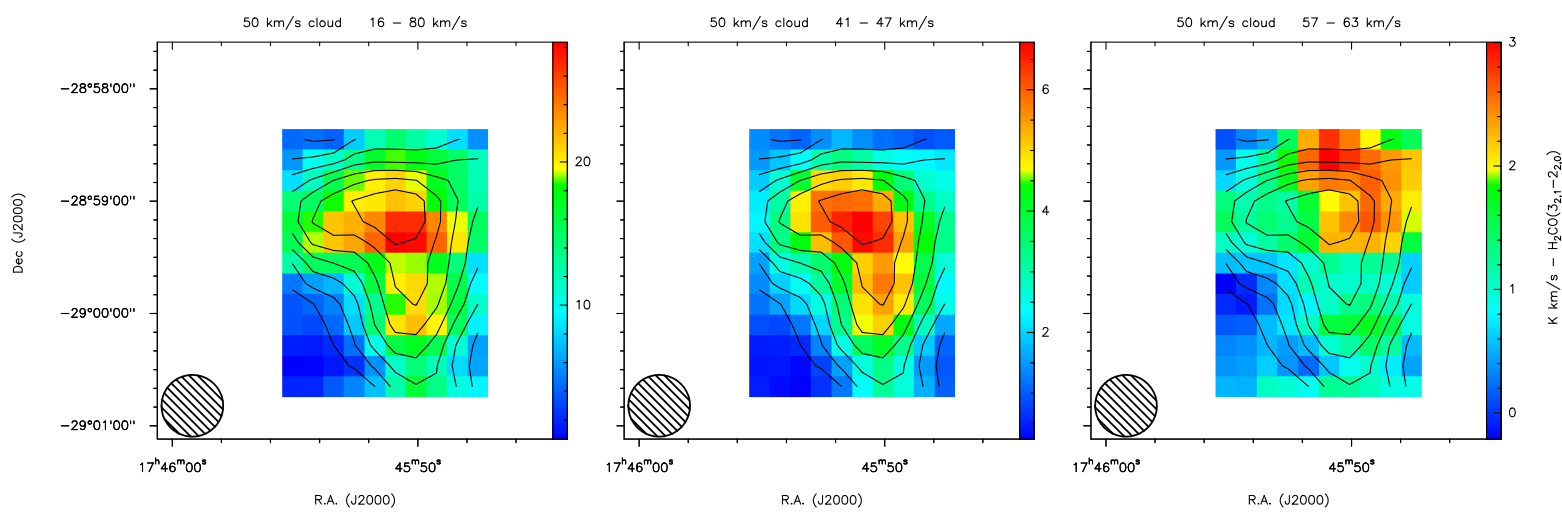

$\mathrm{H}_{2} \mathrm{CO}\left(4_{0,3}-3_{0,3}\right)$
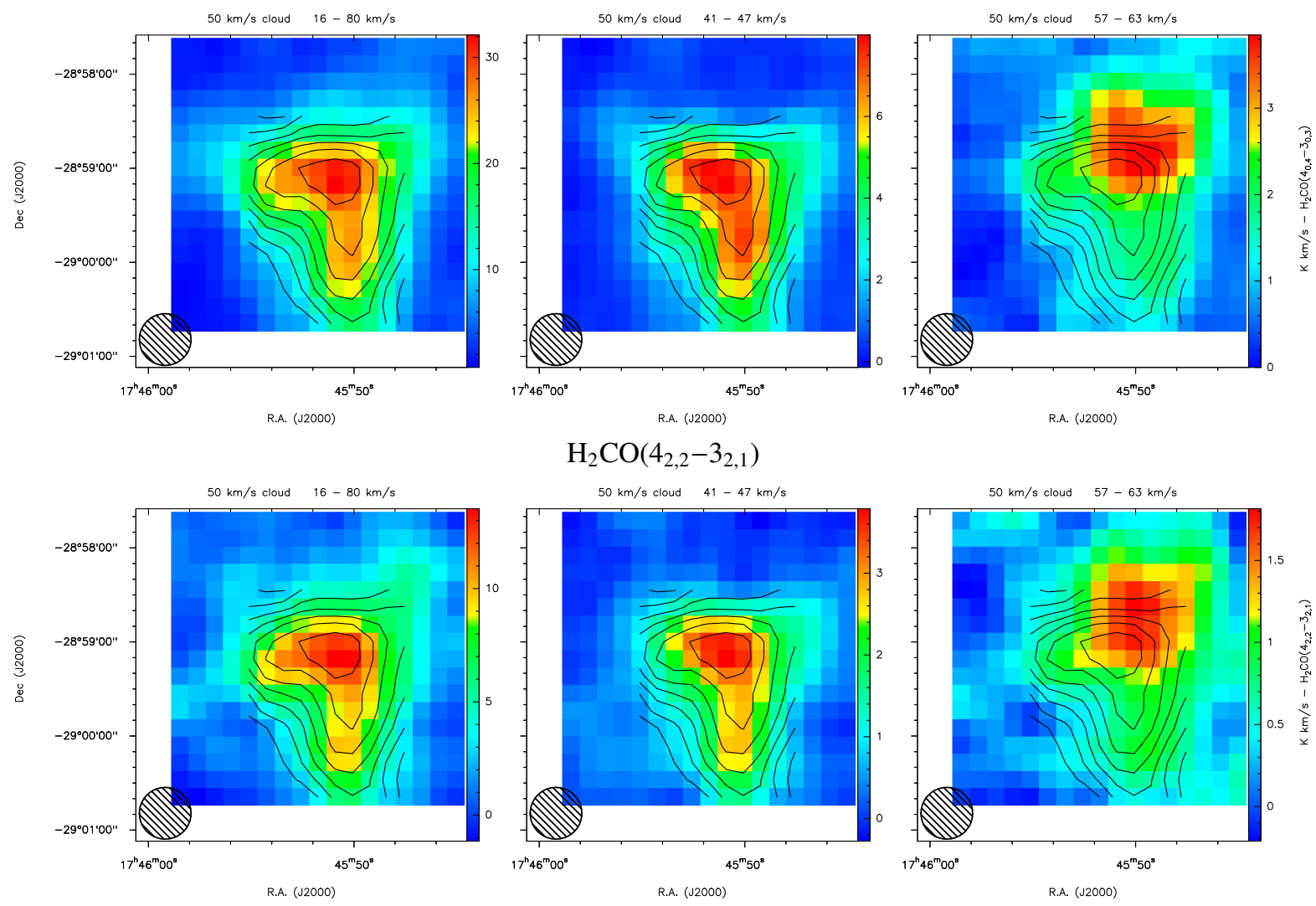

Fig. E.2. As Fig. E. 1 for the $50 \mathrm{~km} \mathrm{~s}^{-1}$ cloud. 
K. Immer et al.: Temperature structures in Galactic center clouds
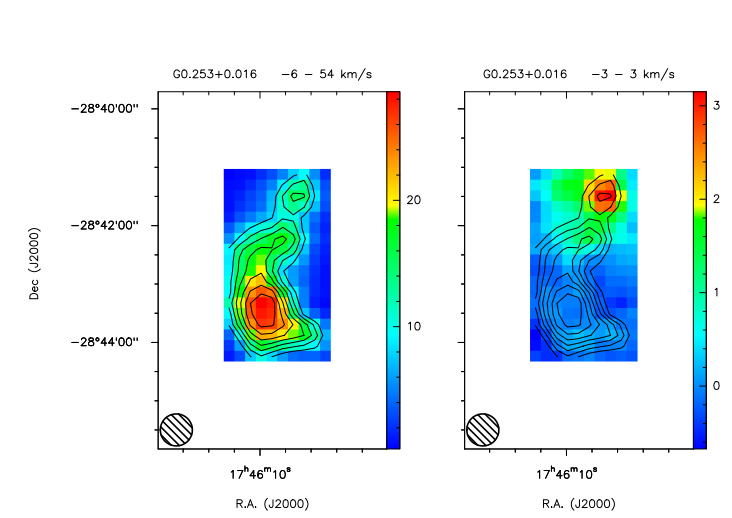

$$
\mathrm{H}_{2} \mathrm{CO}\left(3_{0,3}-2_{0,2}\right)
$$
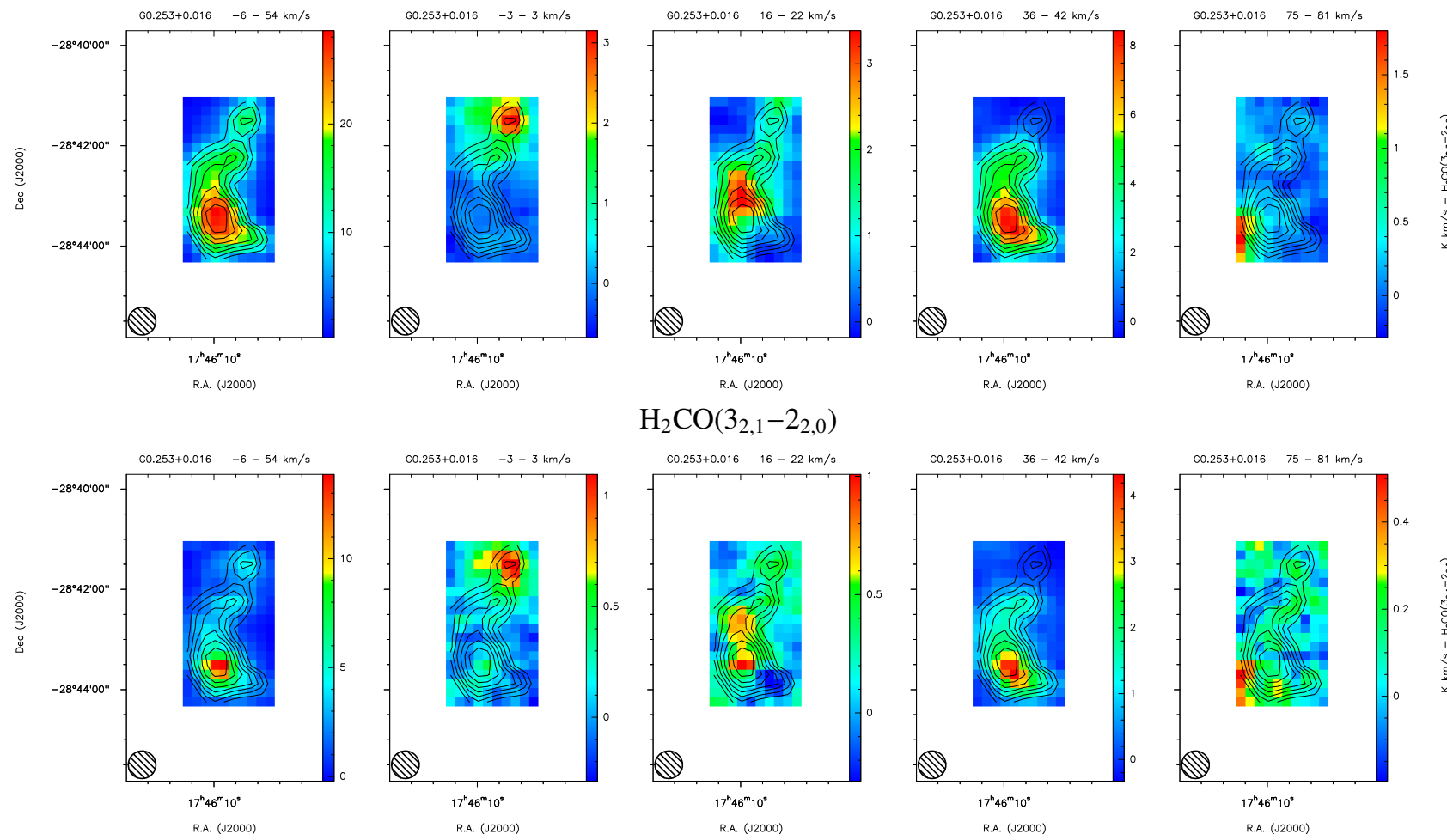

$\mathrm{H}_{2} \mathrm{CO}\left(3_{2,1}-2_{2,0}\right)$
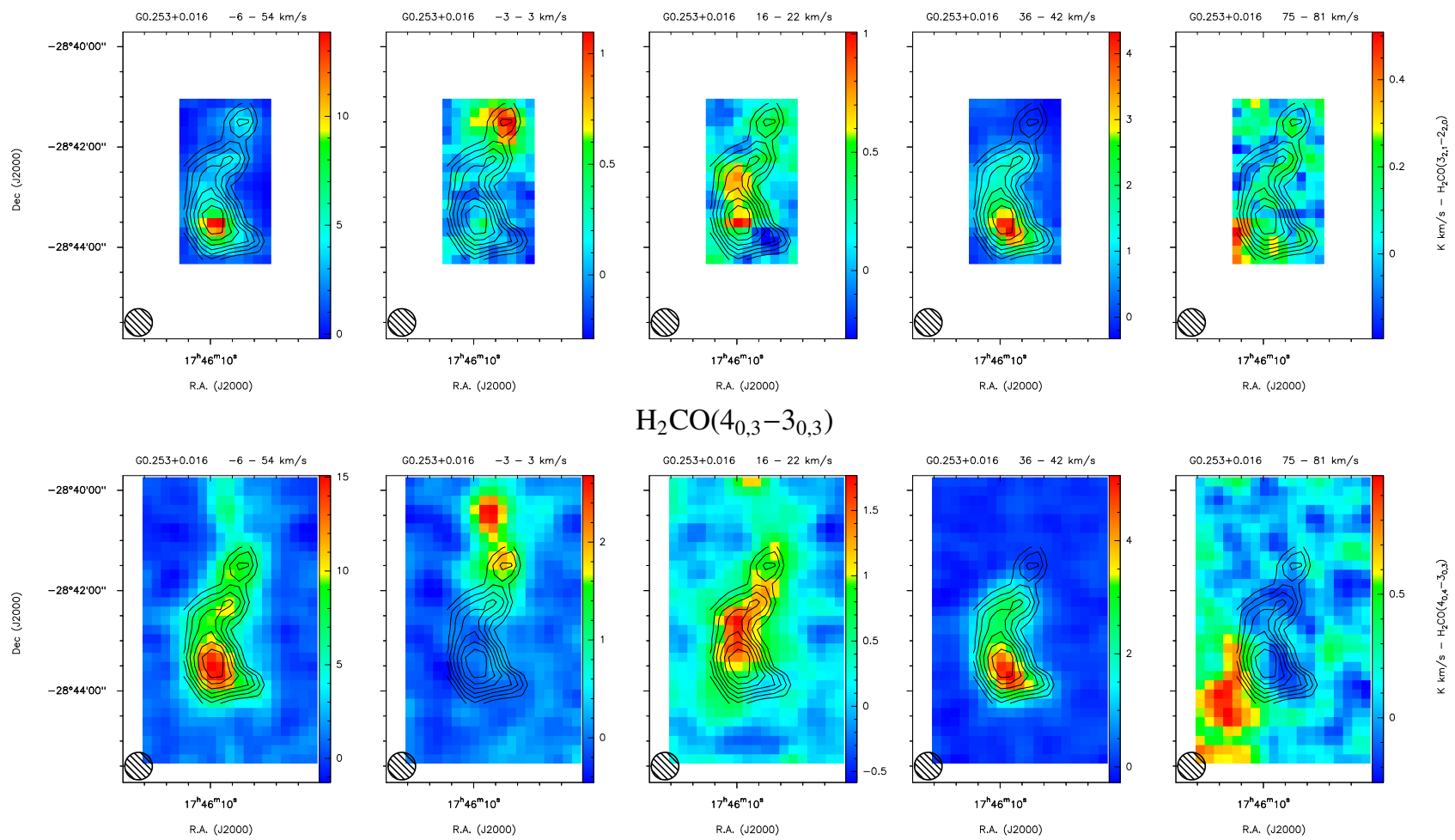

$$
\mathrm{H}_{2} \mathrm{CO}\left(4_{0,3}-3_{0,3}\right)
$$
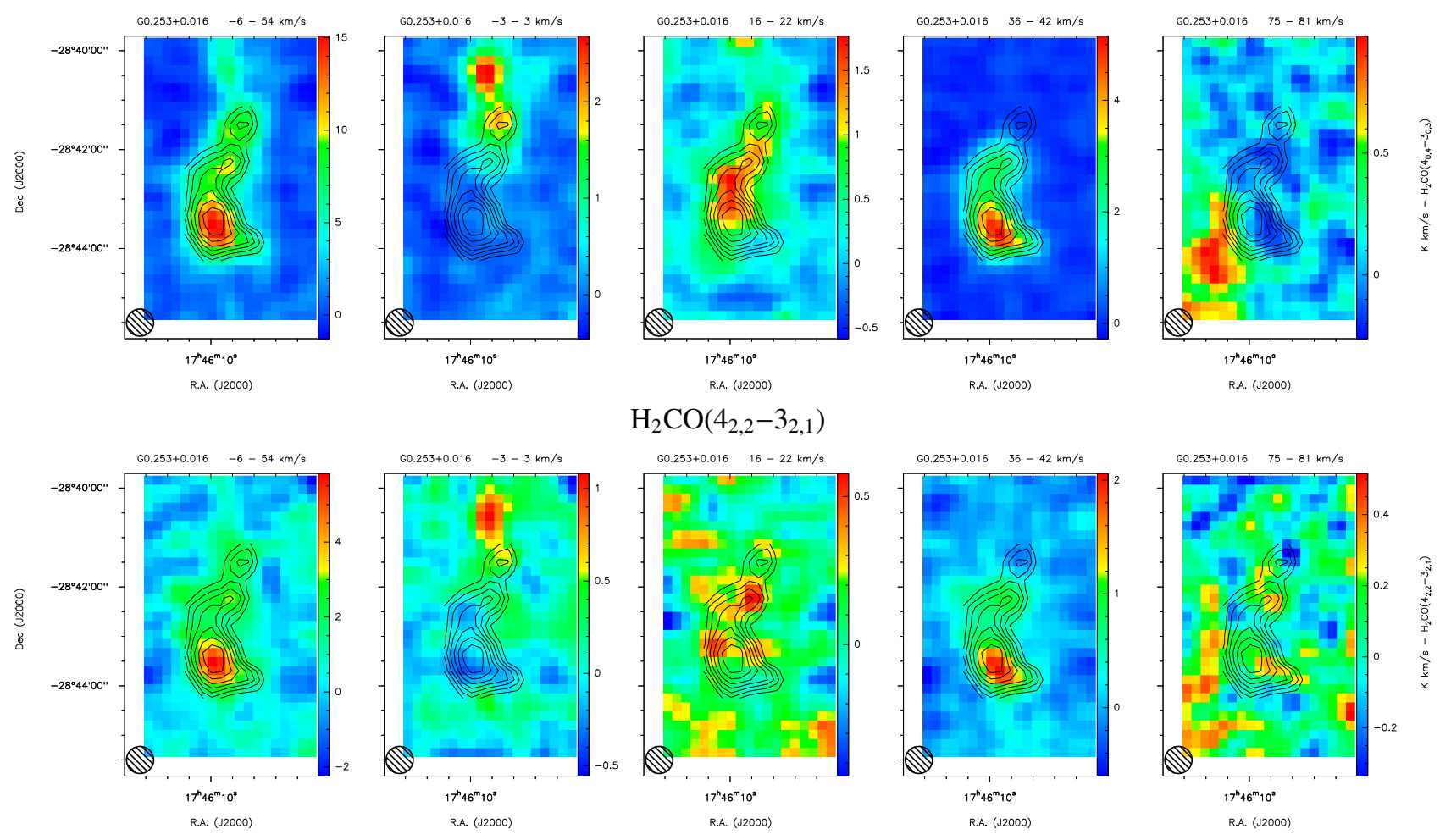

$\mathrm{H}_{2} \mathrm{CO}\left(4_{2,2}-3_{2,1}\right)$
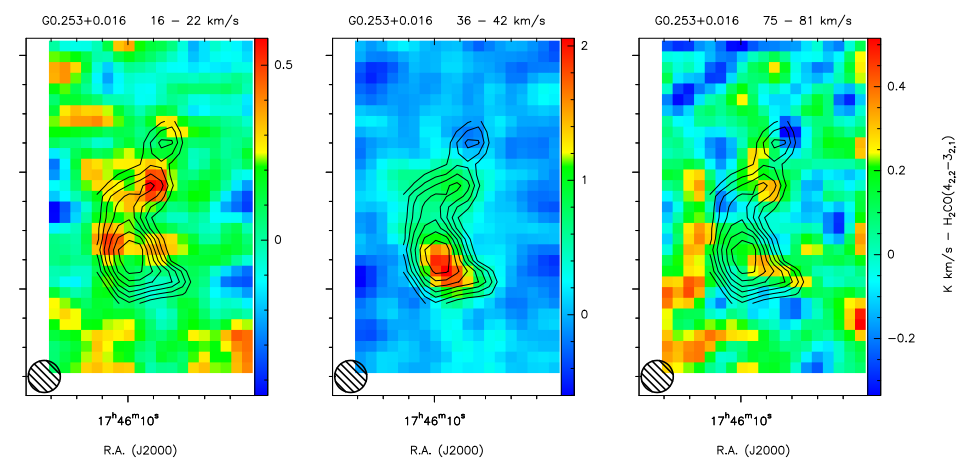

Fig. E.3. As Fig. E.1 for G0.253+0.016. 
A\&A 595, A94 (2016)
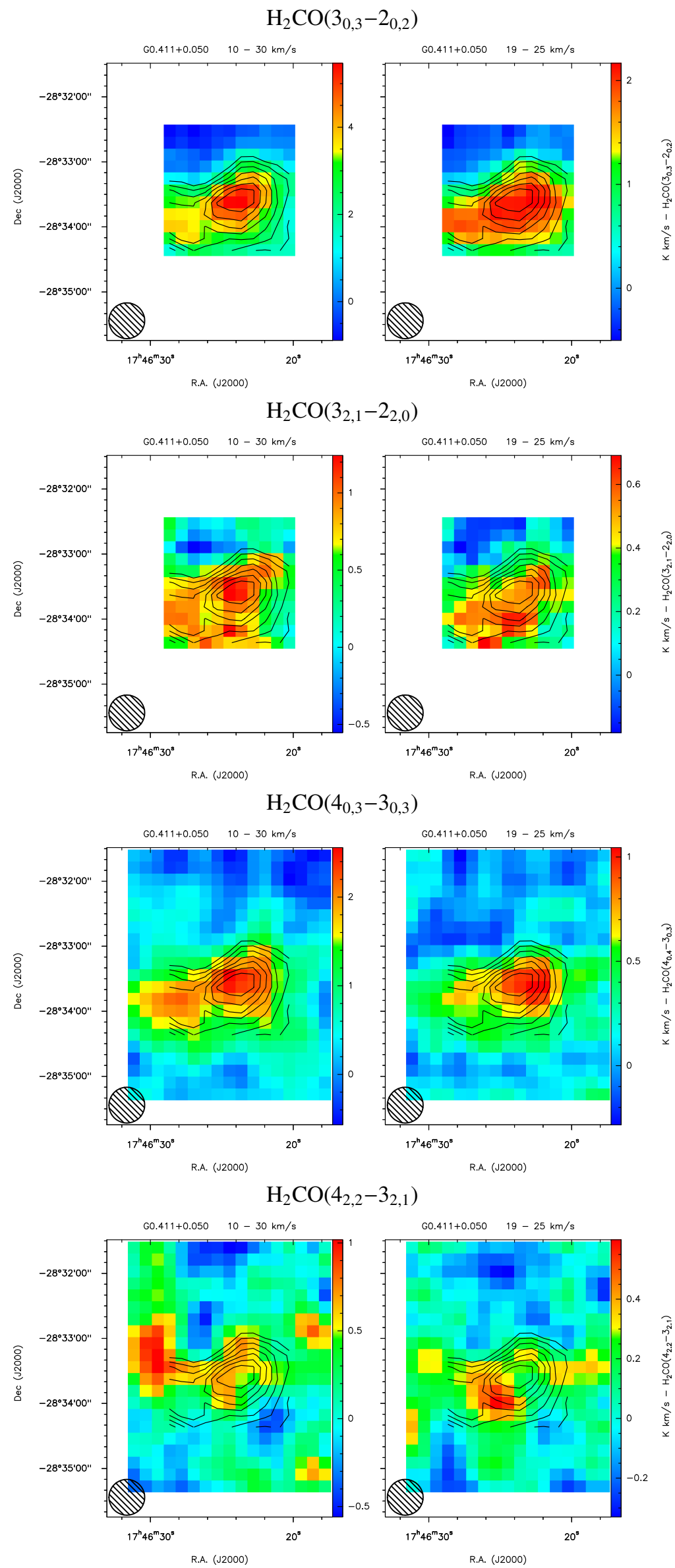

Fig. E.4. As Fig. E.1 for G0.411+0.050. 
K. Immer et al.: Temperature structures in Galactic center clouds
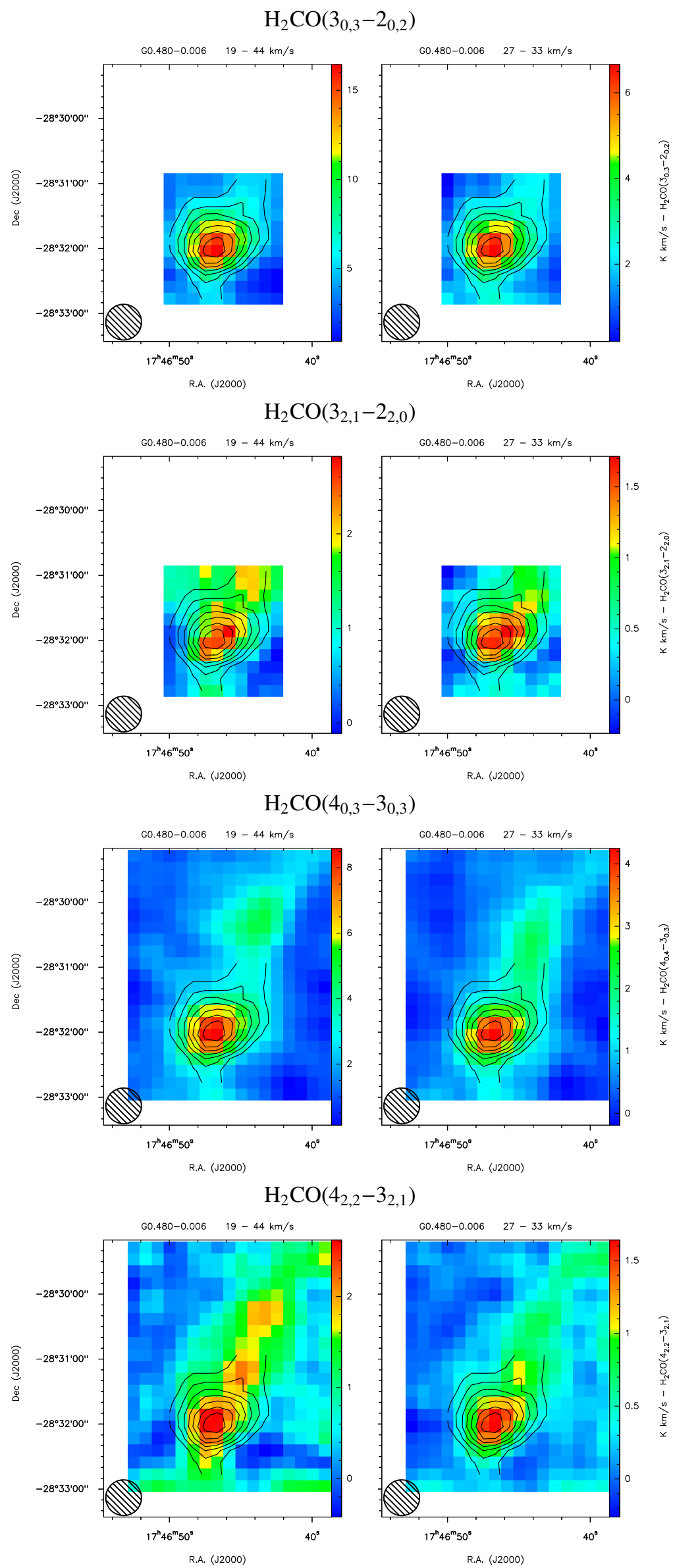

Fig. E.5. As Fig. E.1 for G0.480-0.006. 
A\&A 595, A94 (2016)
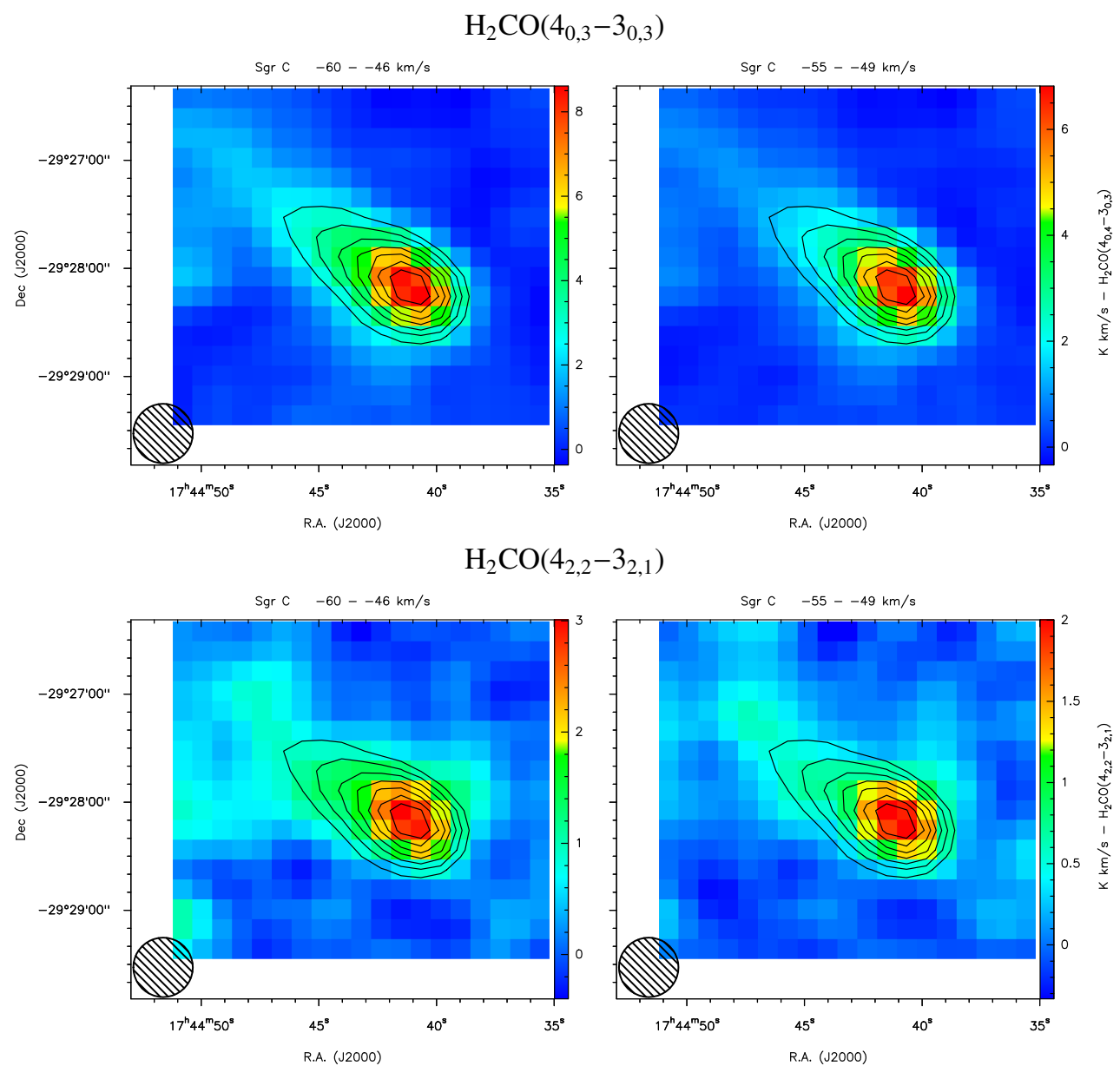

Fig. E.6. As Fig. E.1 for Sgr C. 
K. Immer et al.: Temperature structures in Galactic center clouds
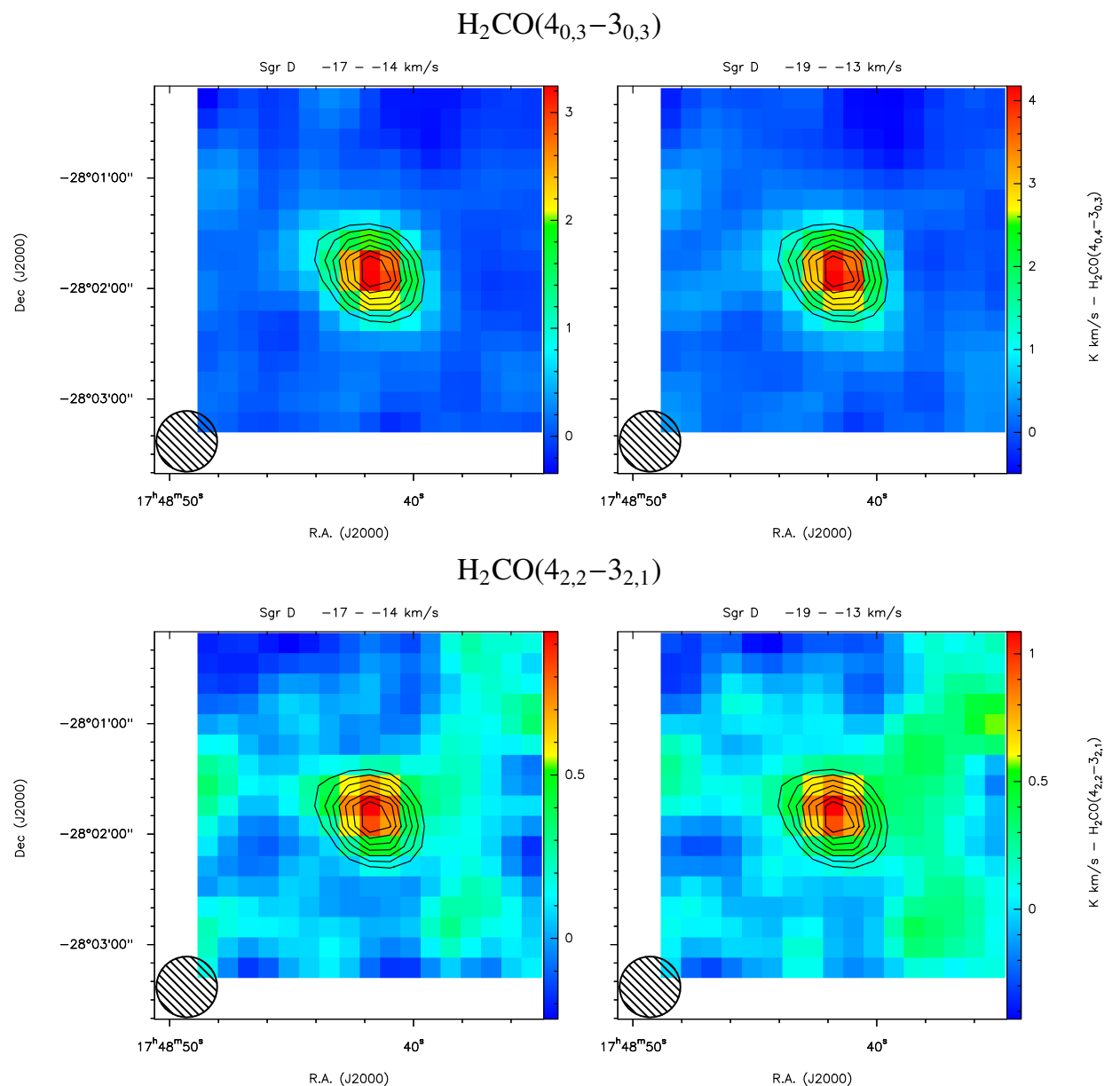

Fig. E.7. As Fig. E.1 for Sgr D. 


\section{Appendix F: Integrated intensity ratio and uncertainty maps}
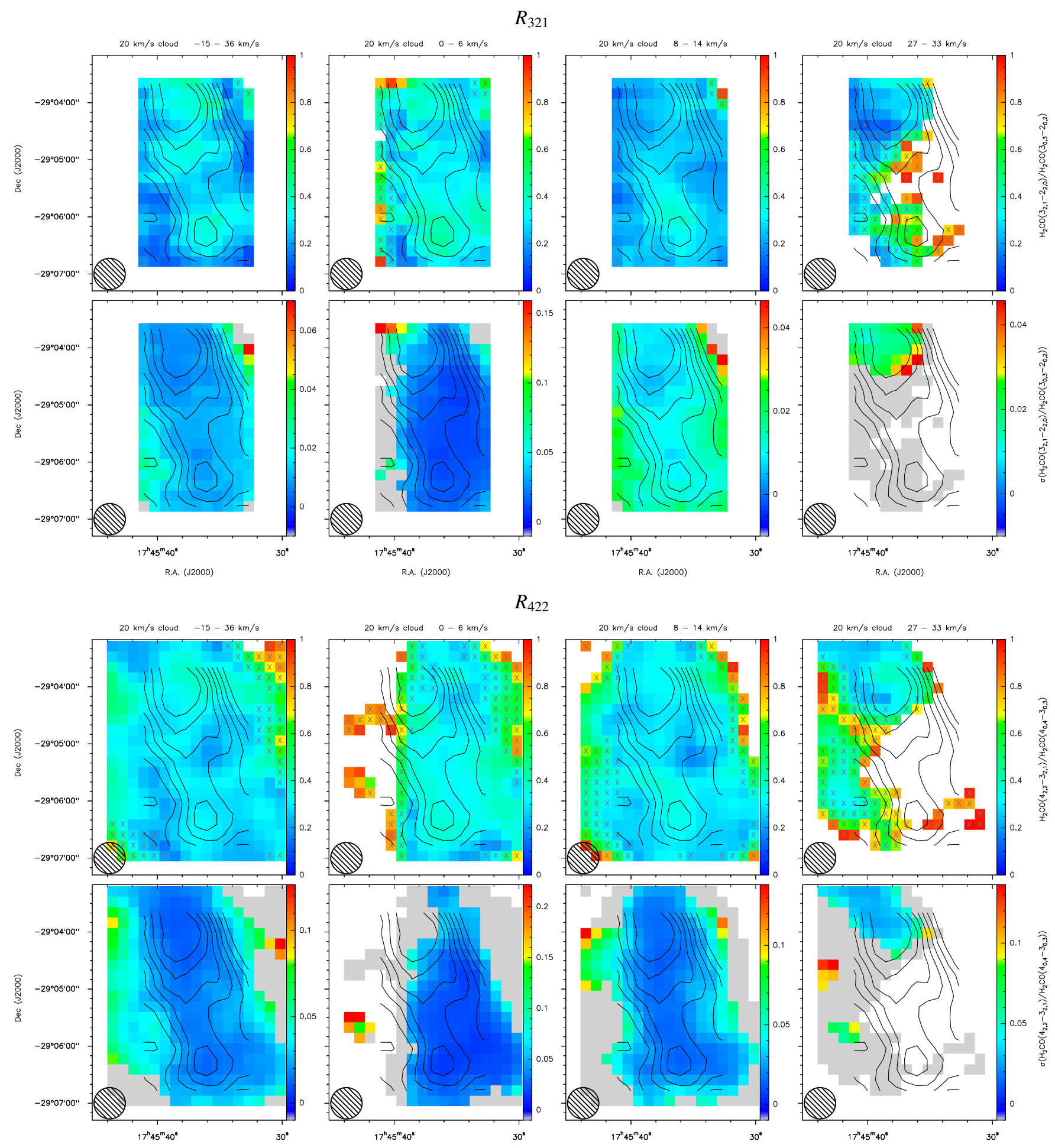

Fig. F.1. Integrated intensity ratio (upper panels) and uncertainty (lower panels) maps of the $20 \mathrm{~km} \mathrm{~s}^{-1}$ cloud (contours as in Fig. E.1). Upper limits of the ratios are indicated with Xs. The corresponding pixels in the uncertainty maps are shown in gray. The circle in the lower left corner shows the $33^{\prime \prime}$ beam. 
K. Immer et al.: Temperature structures in Galactic center clouds

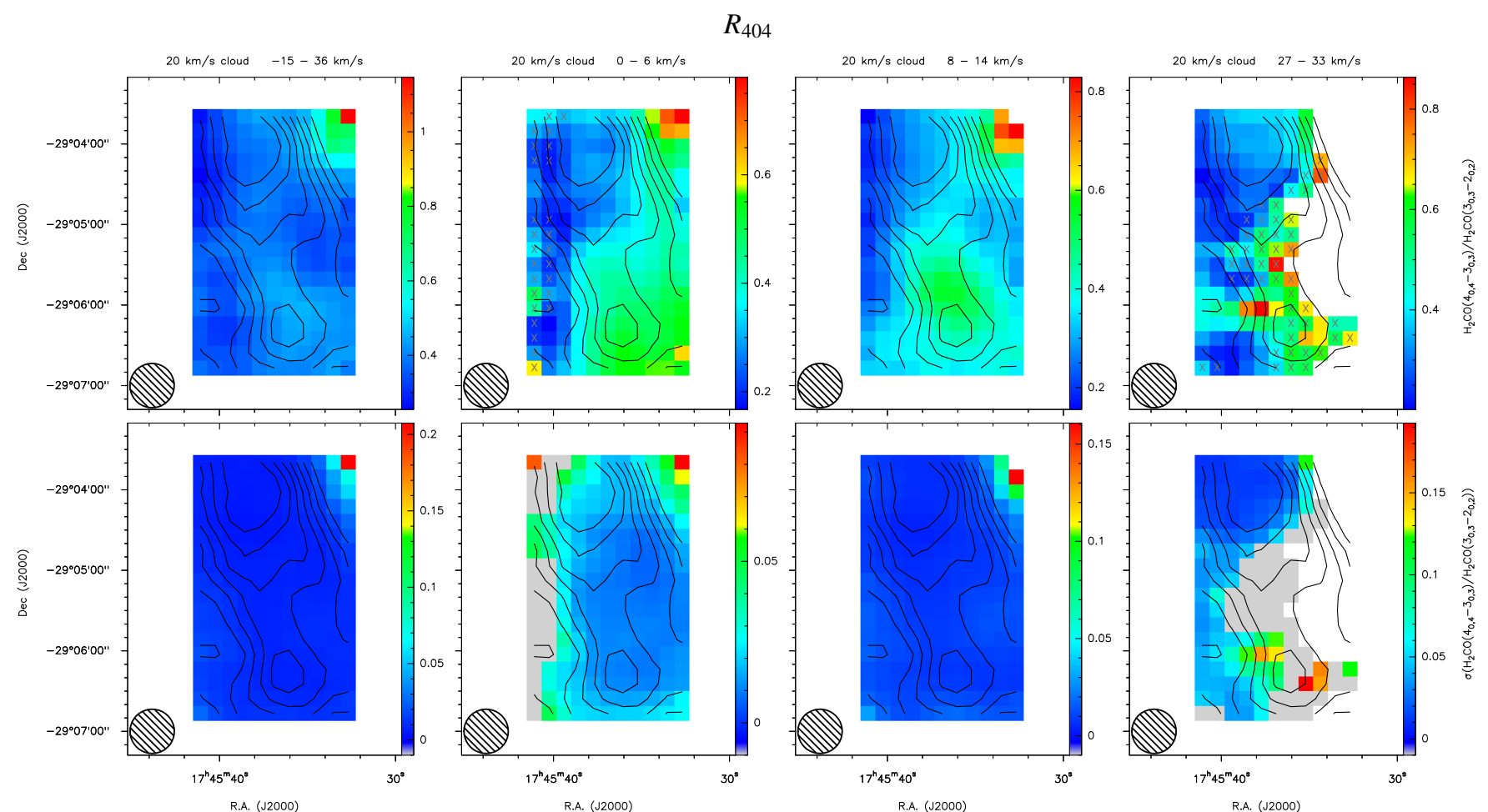

Fig. F.1. continued. 
A\&A 595, A94 (2016)
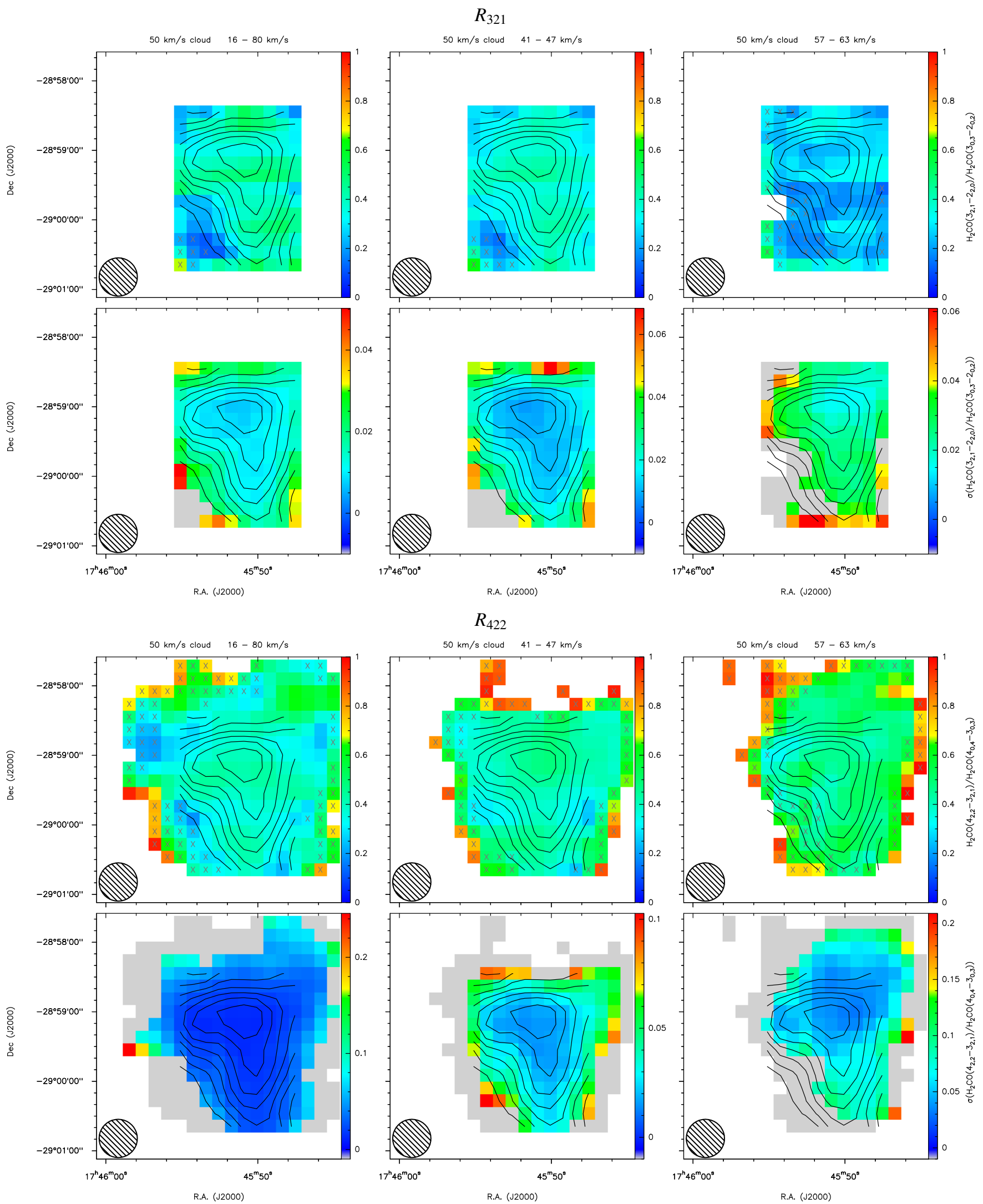

Fig. F.2. As Fig. F.1 for the $50 \mathrm{~km} \mathrm{~s}^{-1}$ cloud. 
K. Immer et al.: Temperature structures in Galactic center clouds
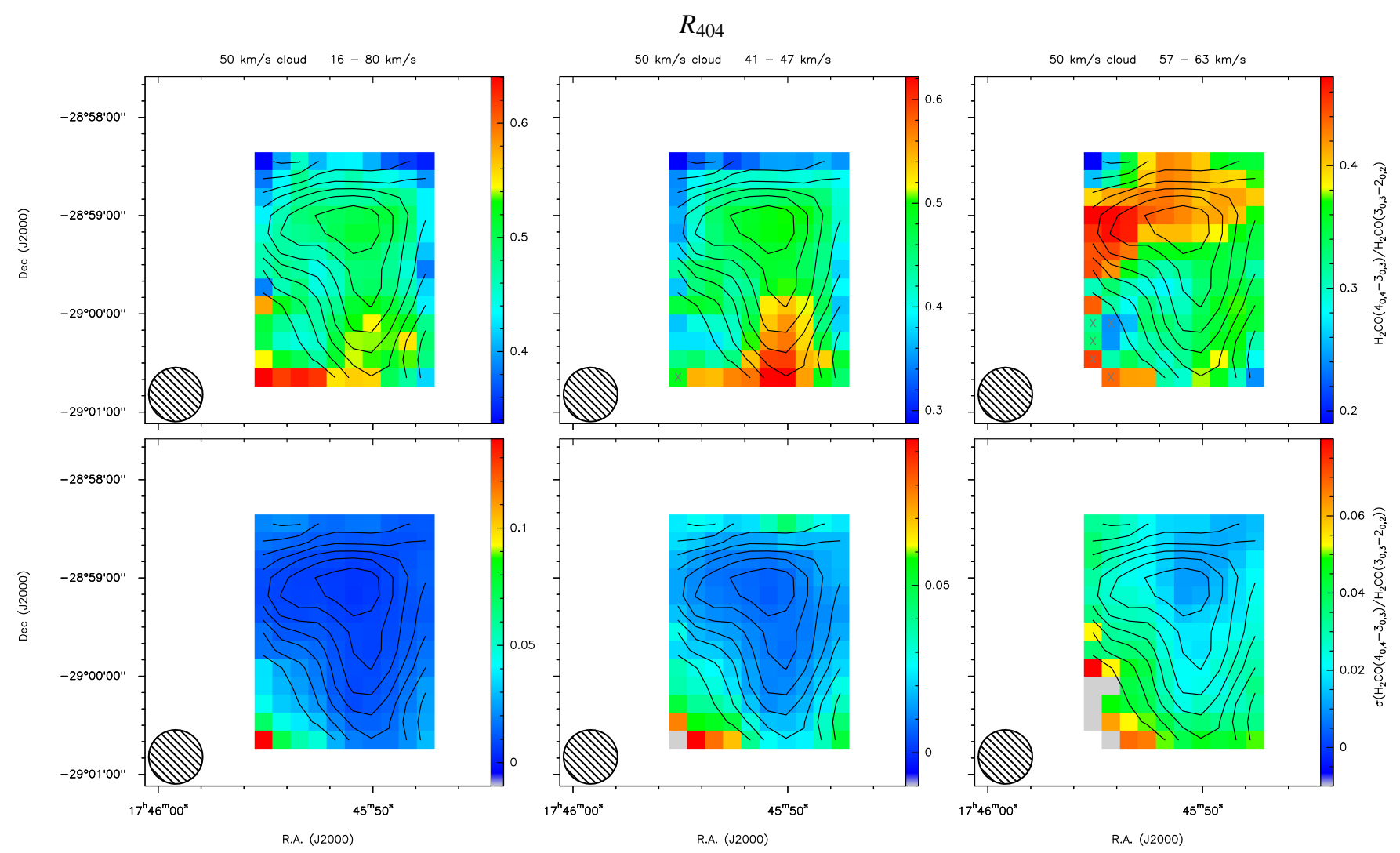

Fig. F.2. continued. 
A\&A 595, A94 (2016)
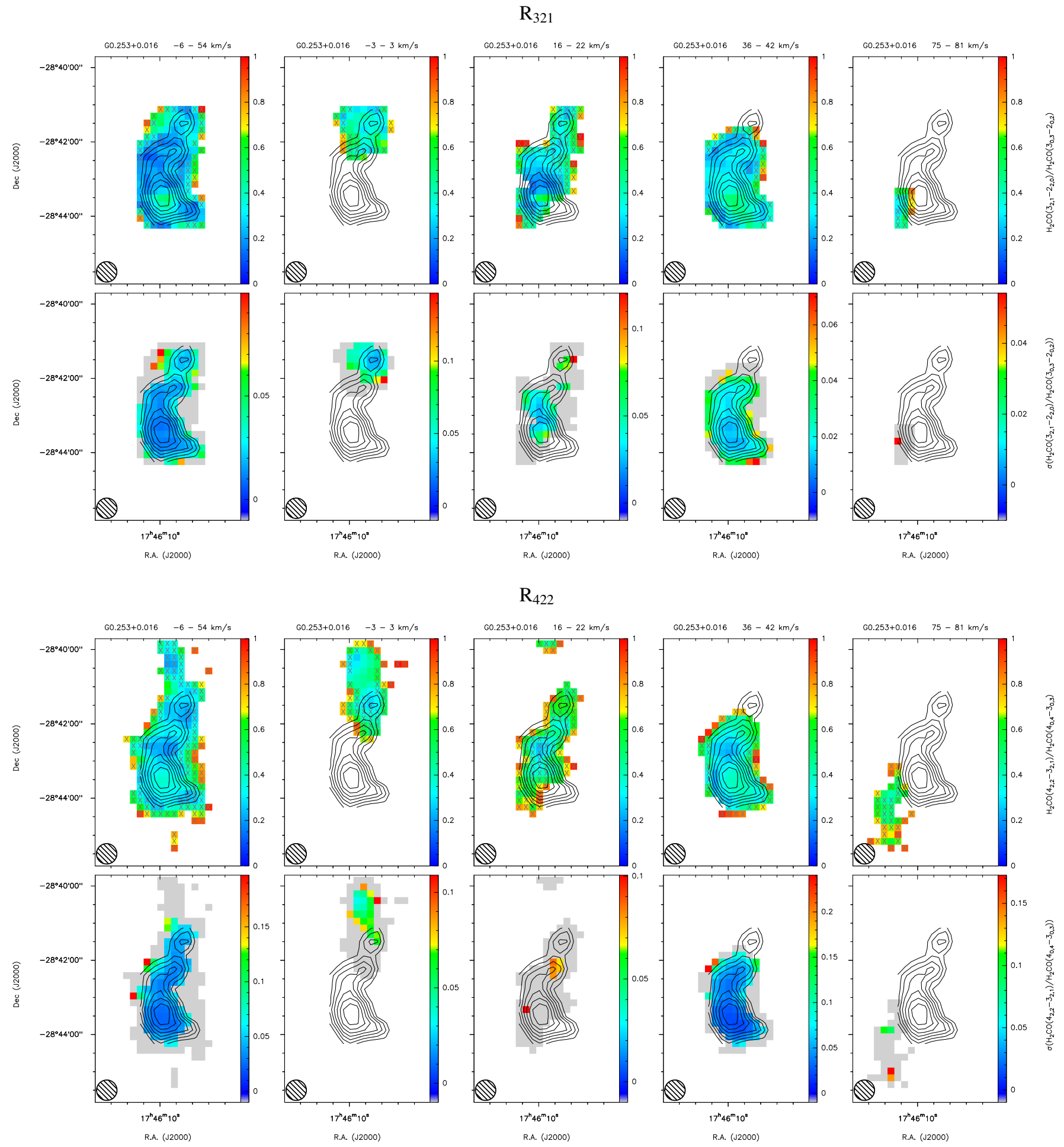

Fig. F.3. As Fig. F.1 for G0.253+0.016. 
K. Immer et al.: Temperature structures in Galactic center clouds

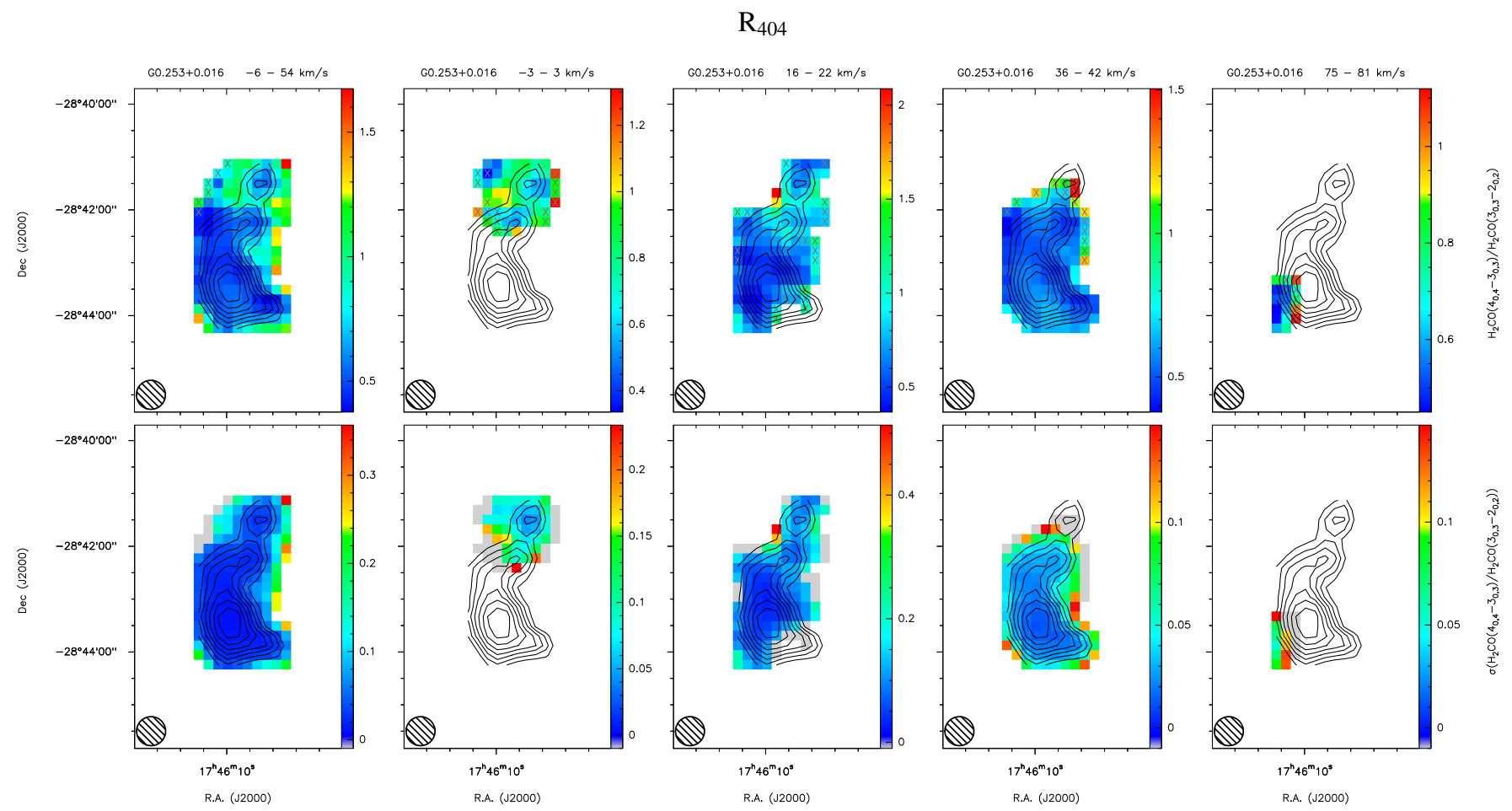

Fig. F.3. continued. 
A\&A 595, A94 (2016)
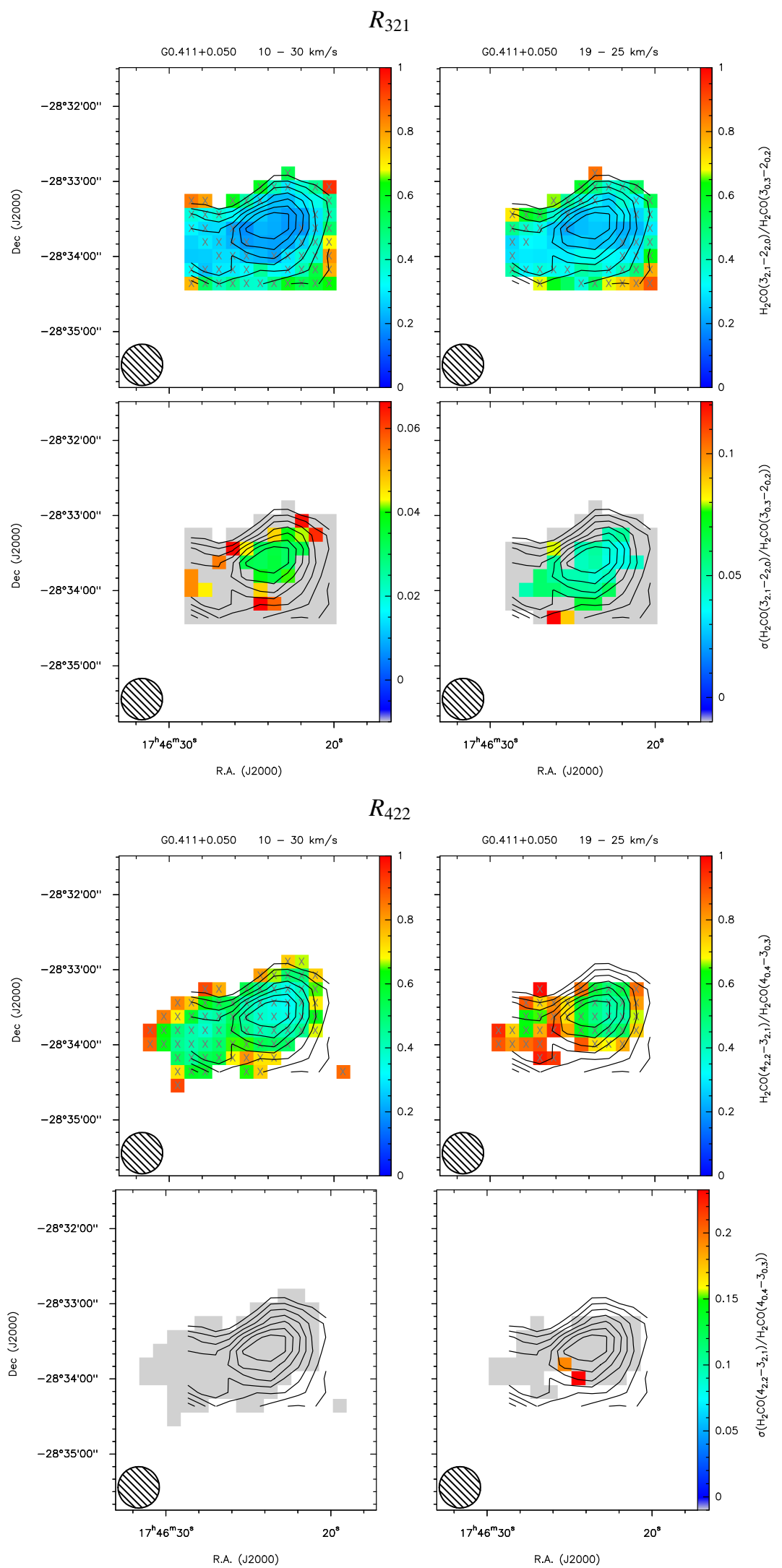

Fig. F.4. As Fig. F.1 for G0.411+0.050. 
K. Immer et al.: Temperature structures in Galactic center clouds

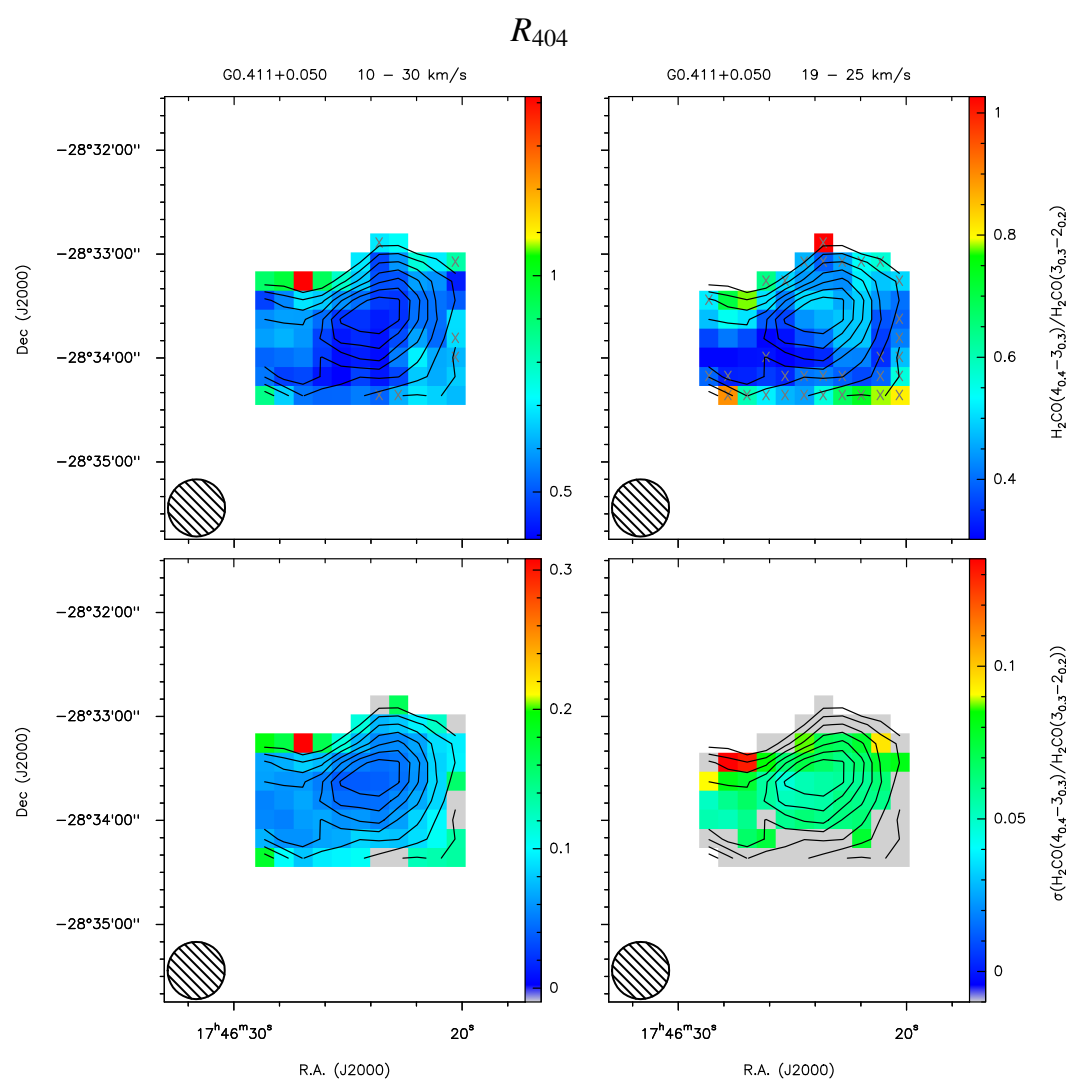

Fig. F.4. continued. 
A\&A 595, A94 (2016)
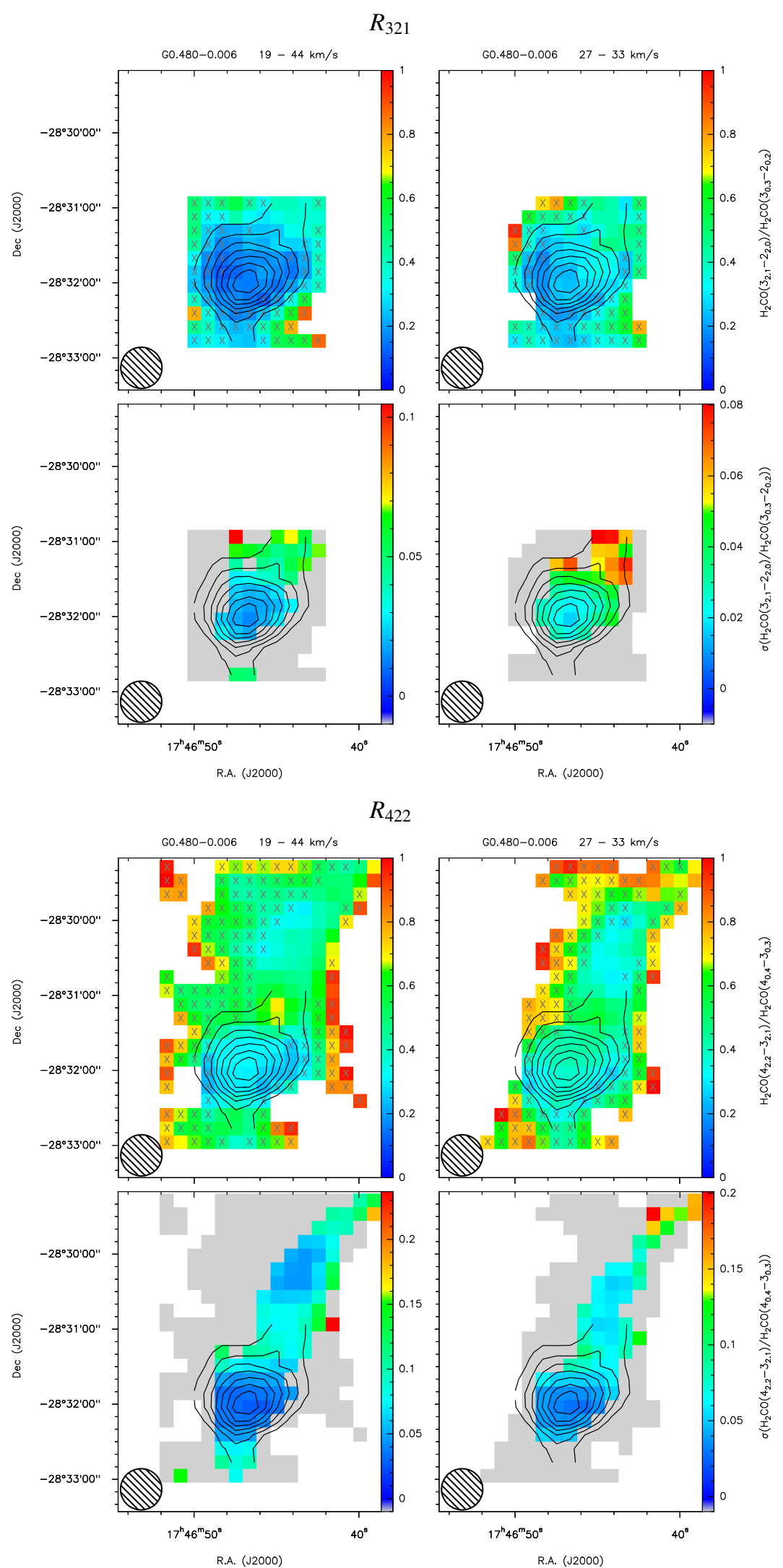

Fig. F.5. As Fig. F.1 for G0.480-0.006. 
K. Immer et al.: Temperature structures in Galactic center clouds

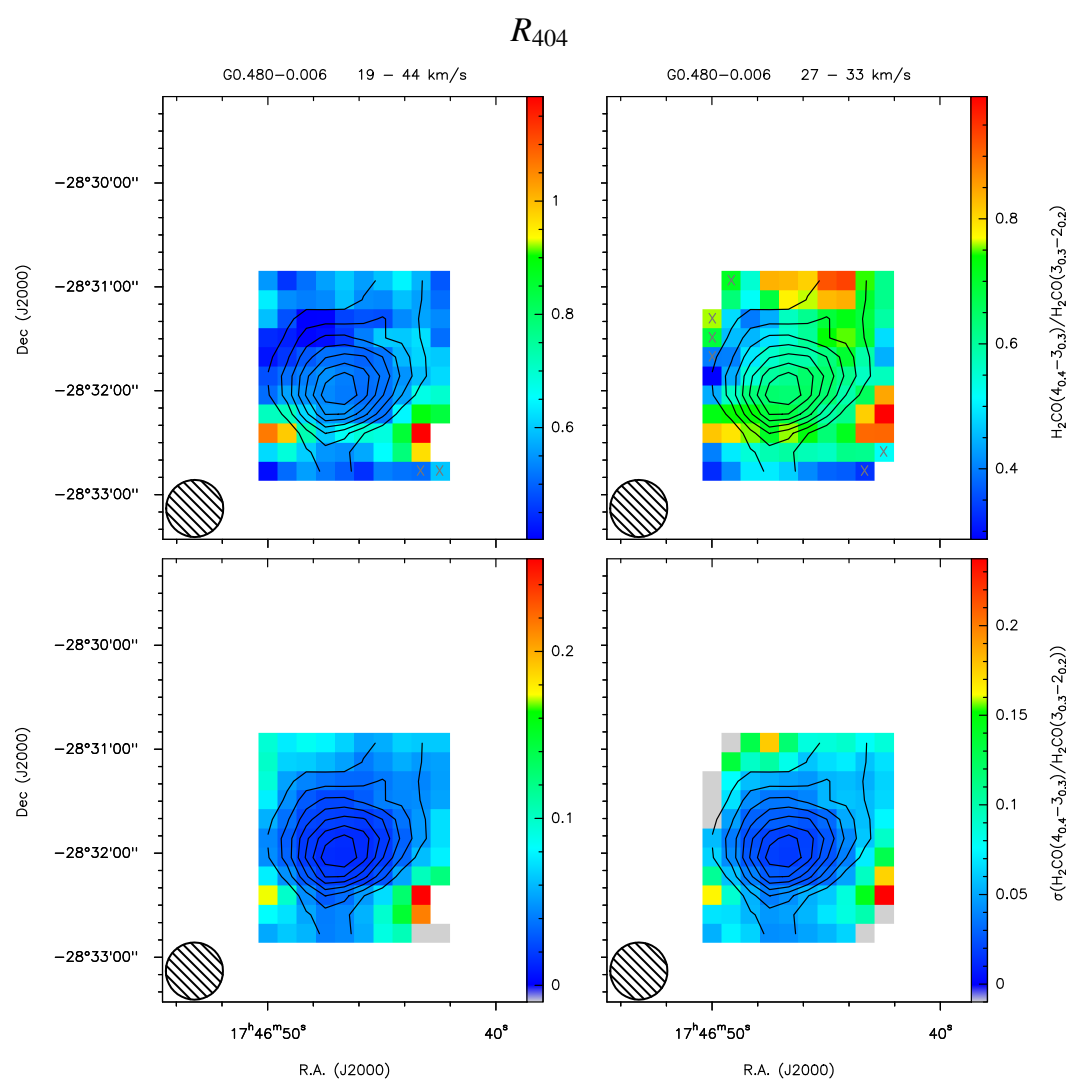

Fig. F.5. continued.
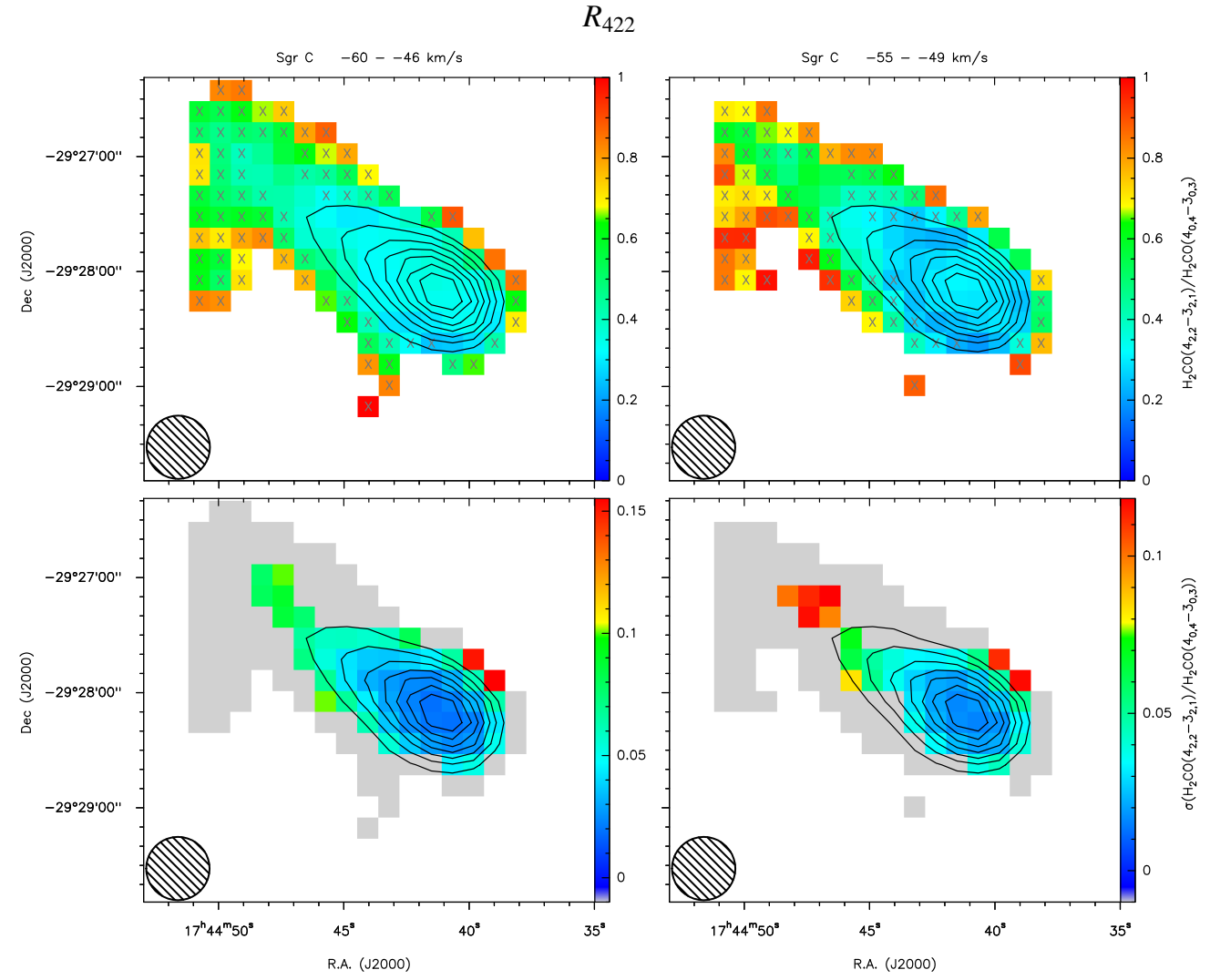

Fig. F.6. As Fig. F.1 for Sgr C. 
A\&A 595, A94 (2016)

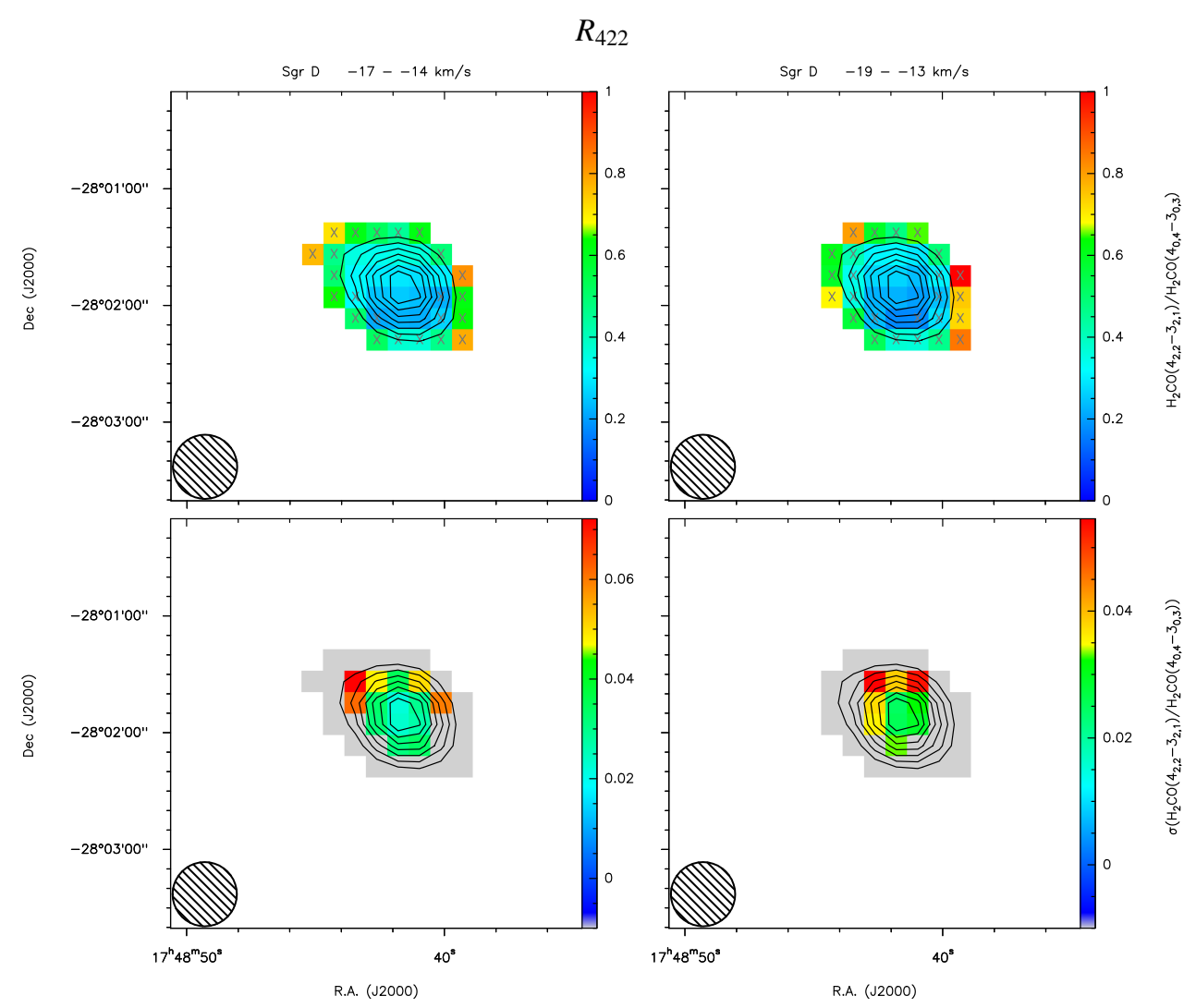

Fig. F.7. As Fig. F.1 for Sgr D. 


\section{Appendix G: Modeling}

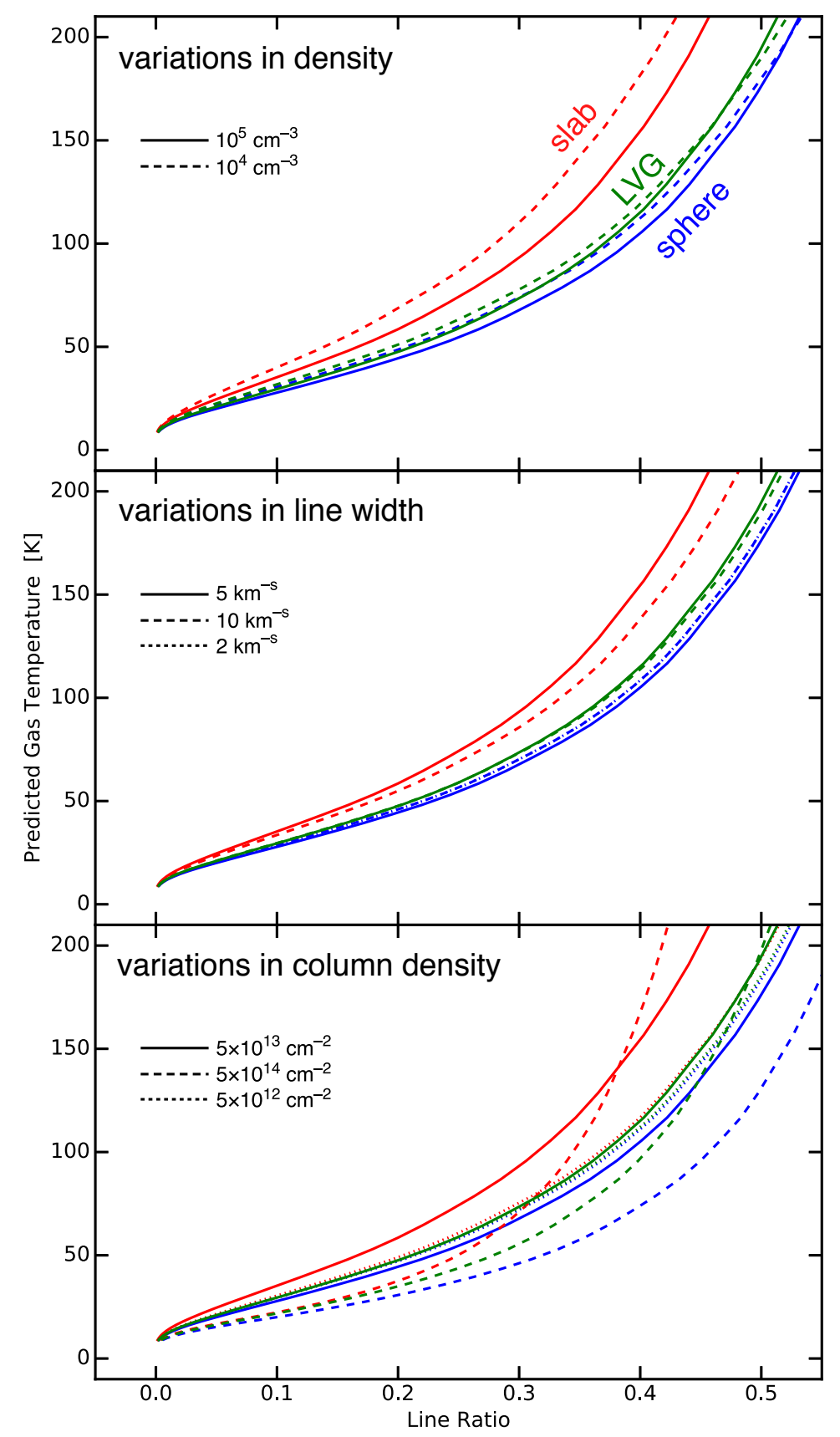

Fig. G.1. Impact of different assumptions in column density, density, line width, and geometry on the estimated temperatures. The solid blue line is alway the fiducial case used to obtain the temperatures presented in this paper. 


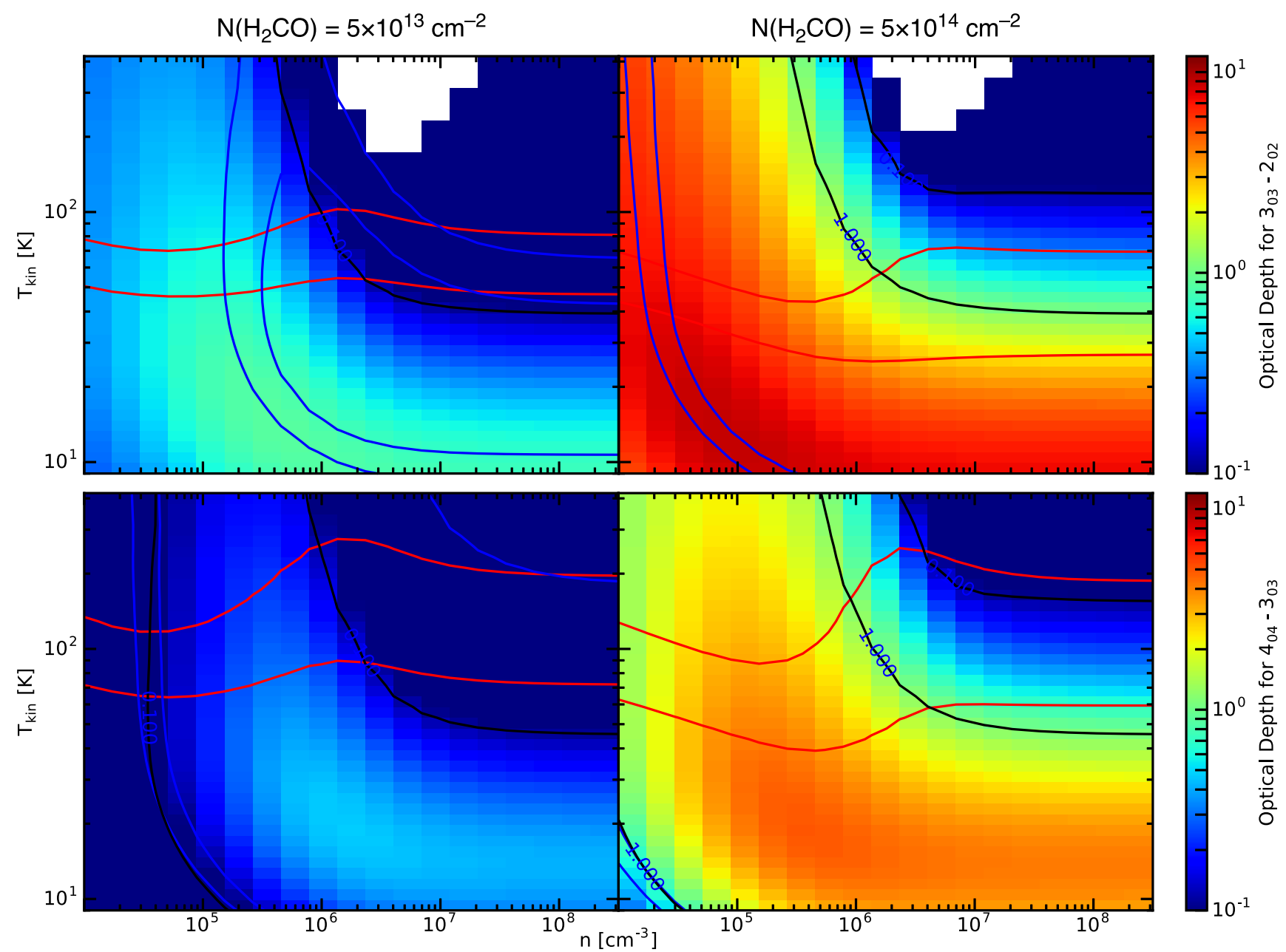

Fig. G.2. Impact of line optical depth. The optical depths shown on the left are calculated for the fiducial case (see Sect. 4.2), while those on the right are for an increased column density of $5 \times 10^{14} \mathrm{~cm}^{-2}$. The red lines indicate the observed line ratios of 0.25 (top panel) and 0.35 (bottom panel) at 218 and $291 \mathrm{GHz}$, respectively, in the $8-14 \mathrm{~km} \mathrm{~s}^{-1}$ slice of the $20 \mathrm{~km} \mathrm{~s}^{-1}$ cloud. The blue lines indicate an intensity of $15 \mathrm{~K} \mathrm{~km} \mathrm{~s}{ }^{-1}$ at $218 \mathrm{GHz}$ (top panel) and of $4 \mathrm{~K} \mathrm{~km} \mathrm{~s}^{-1}$ at $291 \mathrm{GHz}$ (bottom panel), respectively, in the same slice. Black lines give optical depth contours. The lines come in pairs since we allow for $\pm 20 \%$ variation in drawing. Simultaneous fits to line ratios and intensities are found in locations where the blue and red lines intersect. At $218 \mathrm{GHz}$ (top panel) these simultaneous matches require either relatively high densities (left panel) or high optical depths (right panel). 
K. Immer et al.: Temperature structures in Galactic center clouds

Appendix H: Temperature maps

$218 \mathrm{GHz}$ temperatures
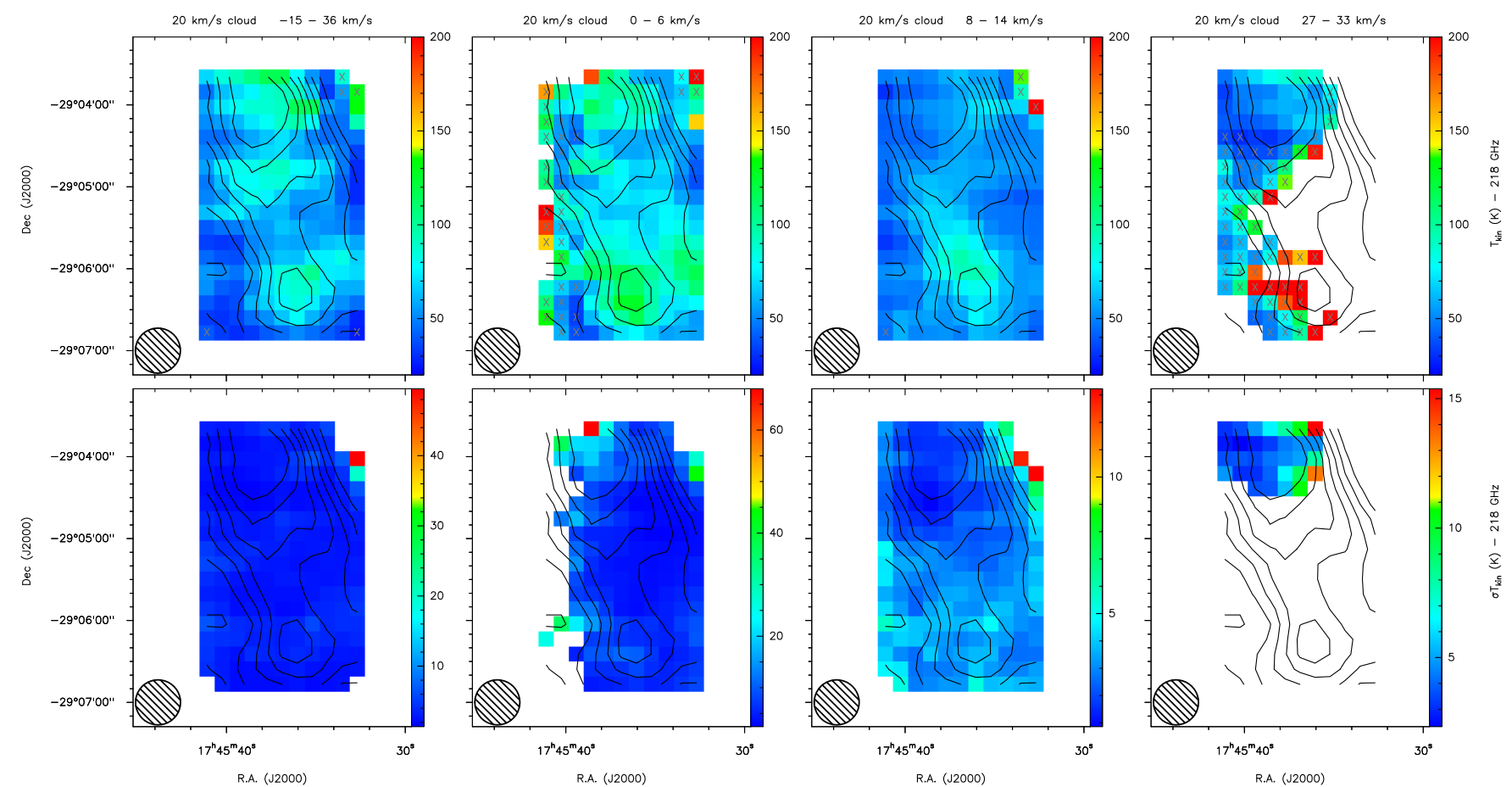

$291 \mathrm{GHz}$ temperatures
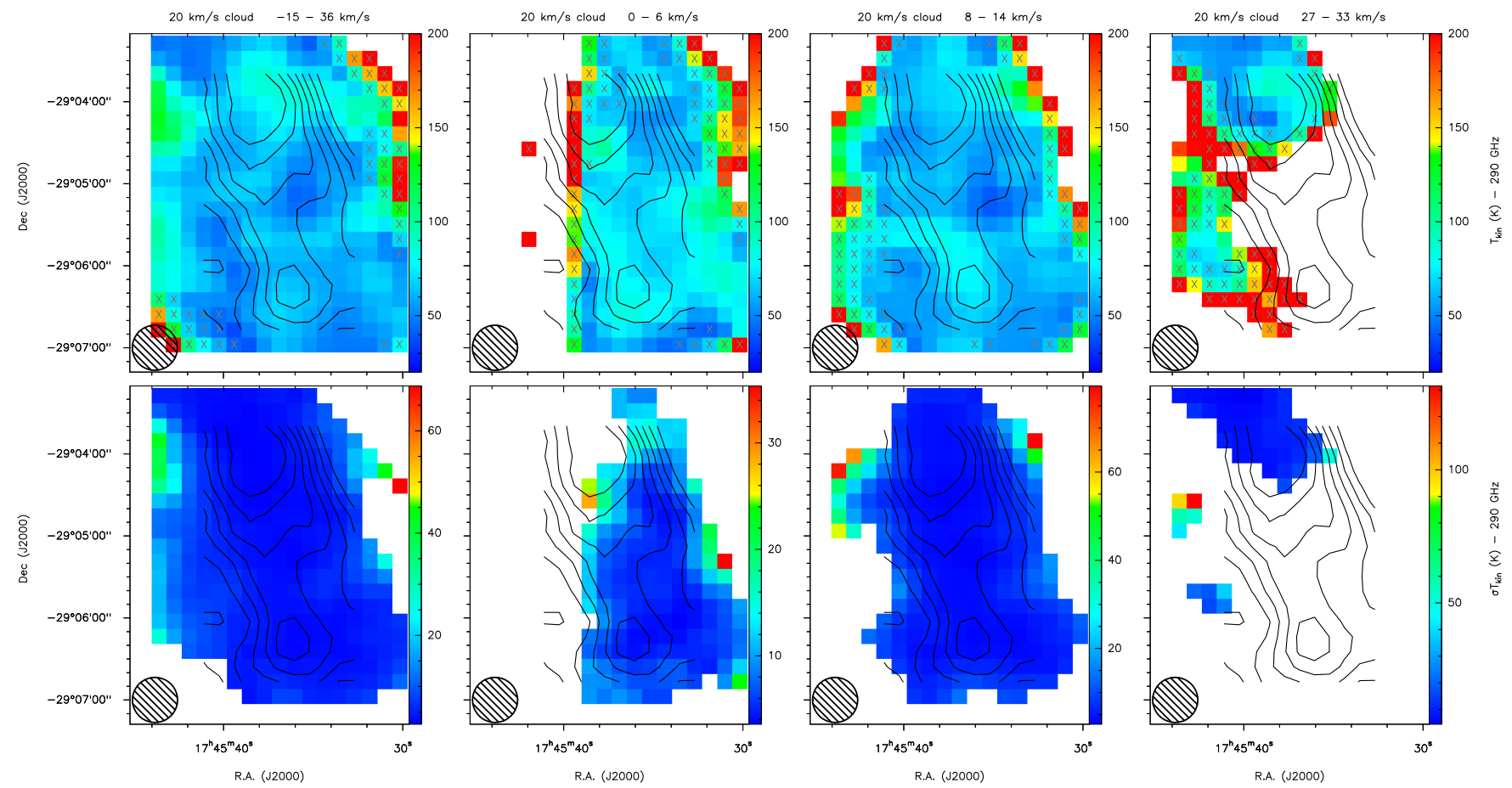

Fig. H.1. Temperature (upper panels) and uncertainty (lower panels) maps of the $20 \mathrm{~km} \mathrm{~s}^{-1}$ cloud (contours as in Fig. E.1). Upper limits of the temperatures are marked with Xs. The corresponding pixels in the uncertainty maps are blanked. The purple squares present the areas over which the emission of the main $\mathrm{H}_{2} \mathrm{CO}$ line was integrated to determine the line width, as well as the average temperature (see Sect. 5.3). The circle in the lower left corner shows the $33^{\prime \prime}$ beam. 
A\&A 595, A94 (2016)

\section{$218 \mathrm{GHz}$ temperatures}
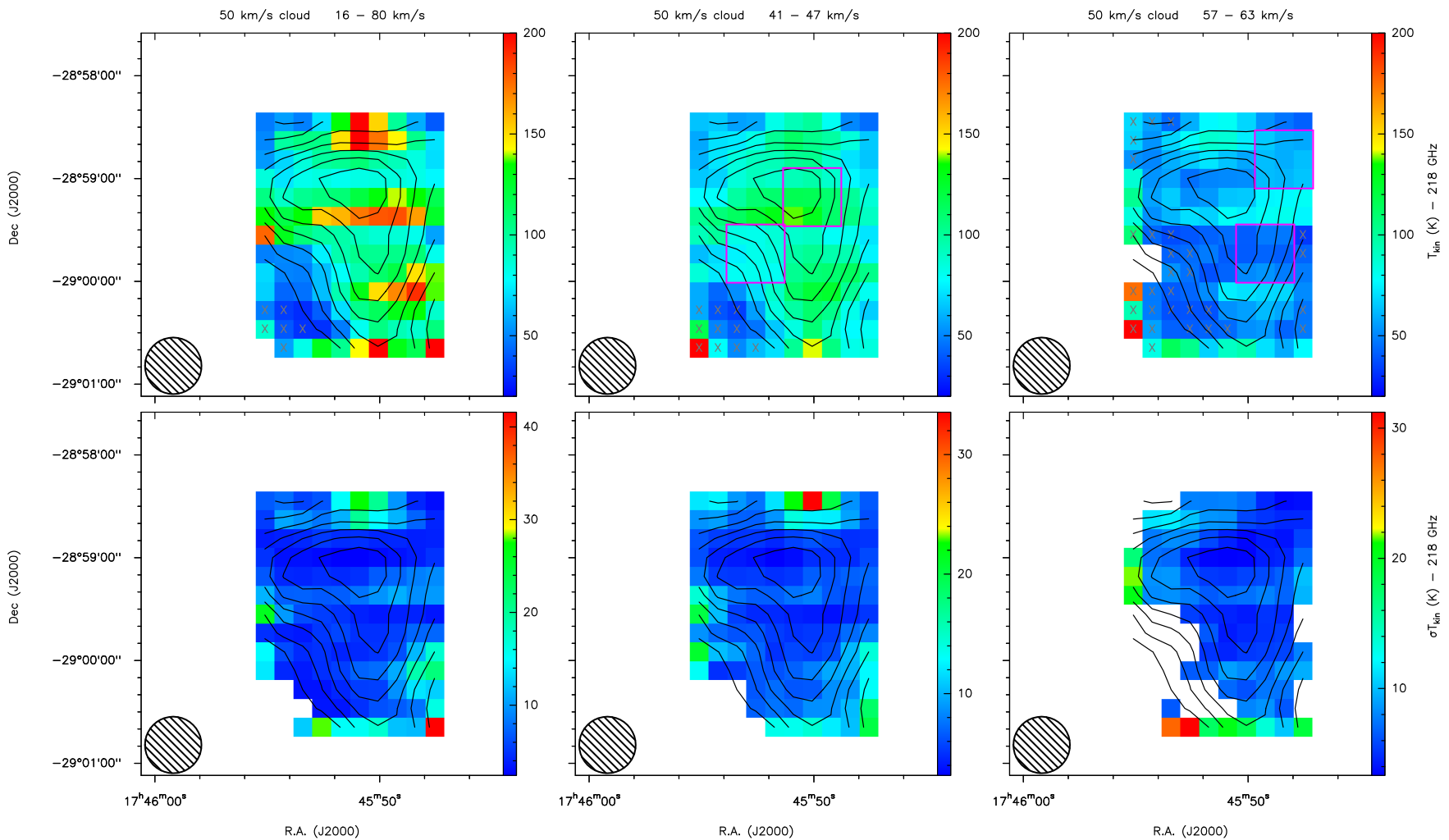

$291 \mathrm{GHz}$ temperatures
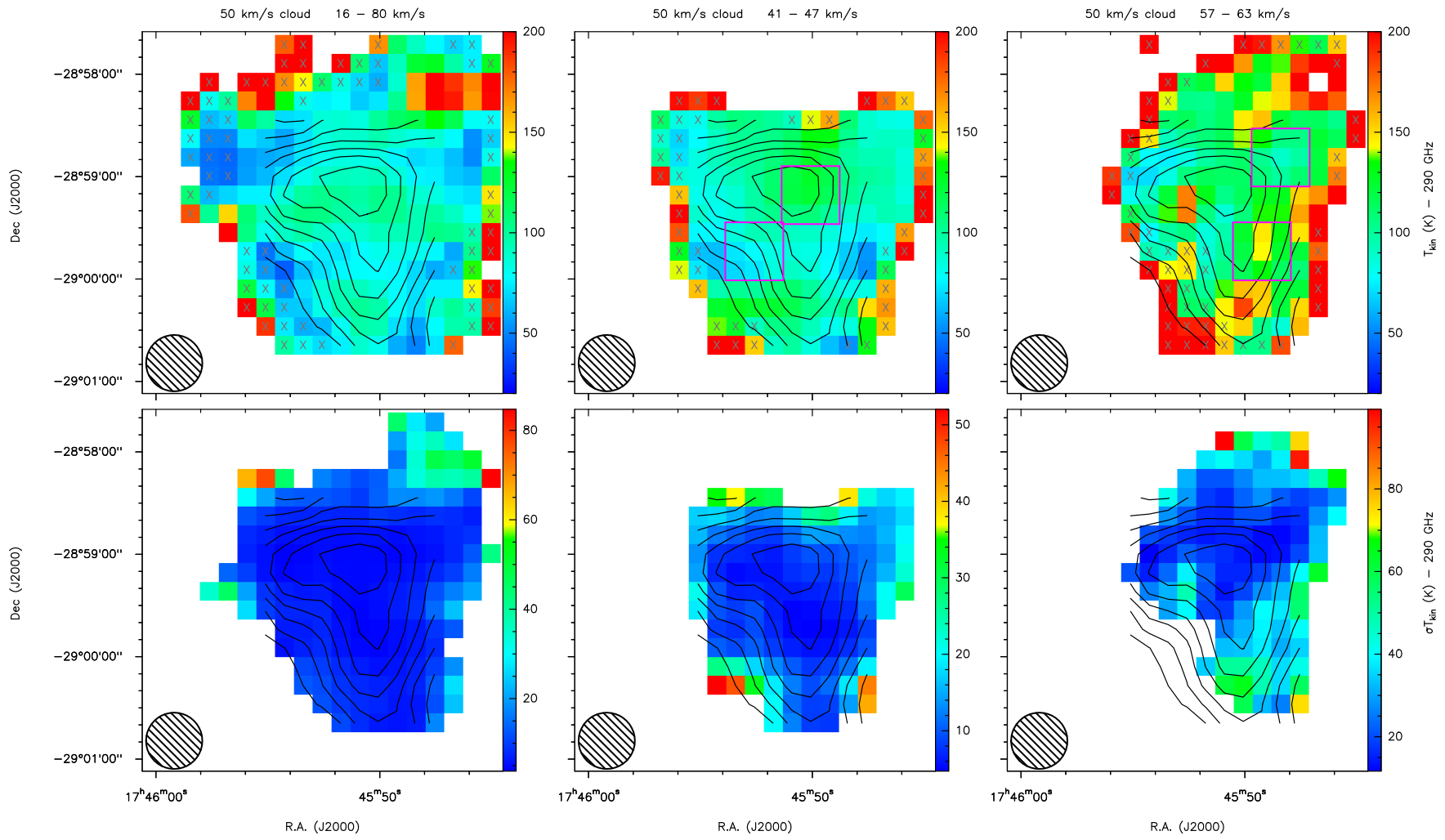

Fig. H.2. As Fig. H.1, for the $50 \mathrm{~km} \mathrm{~s}^{-1}$ cloud. 
K. Immer et al.: Temperature structures in Galactic center clouds

$218 \mathrm{GHz}$ temperatures
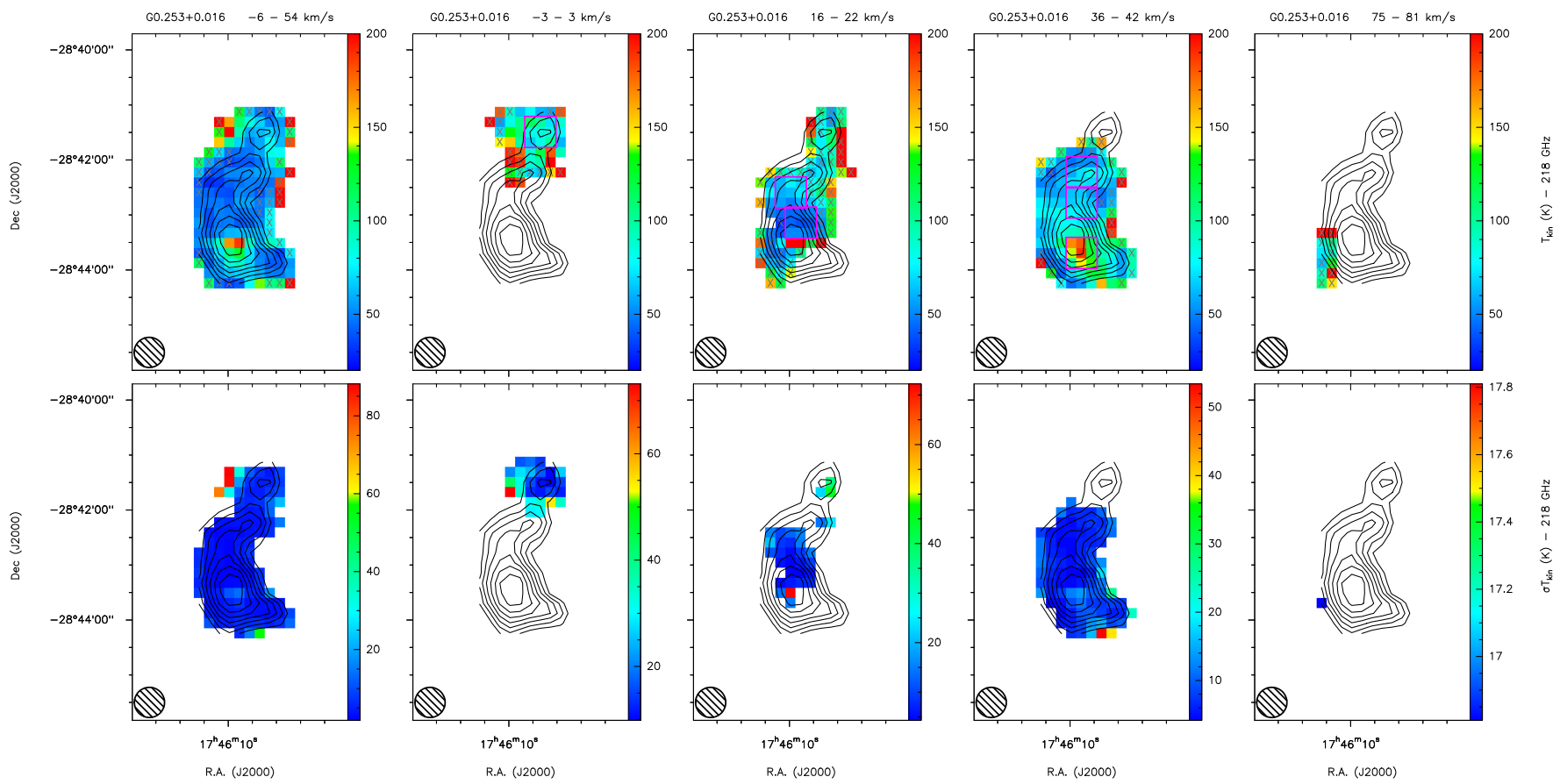

$291 \mathrm{GHz}$ temperatures
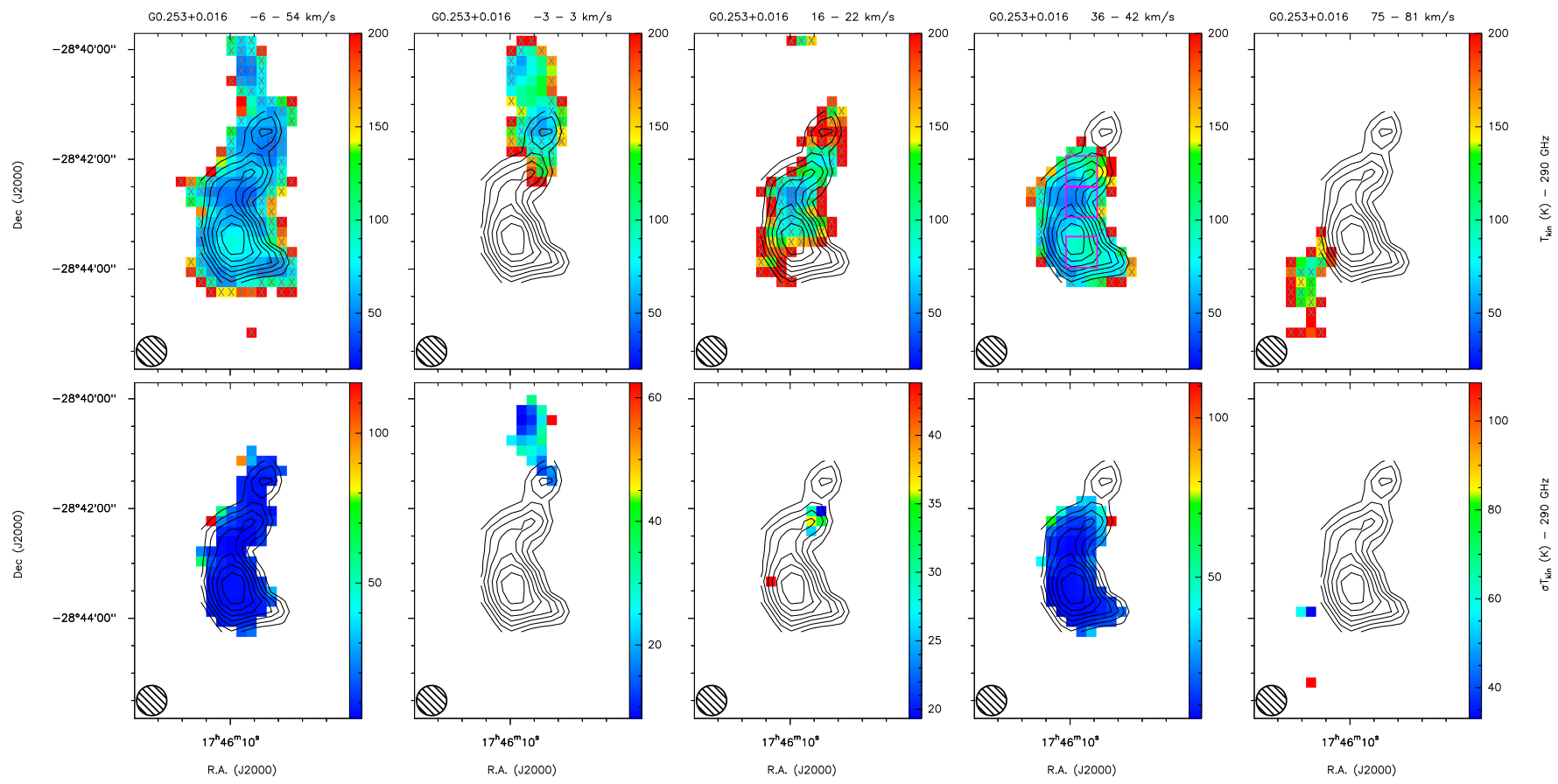

Fig. H.3. As Fig. H.1, for G0.253+0.016. 
A\&A 595, A94 (2016)

$218 \mathrm{GHz}$ temperatures
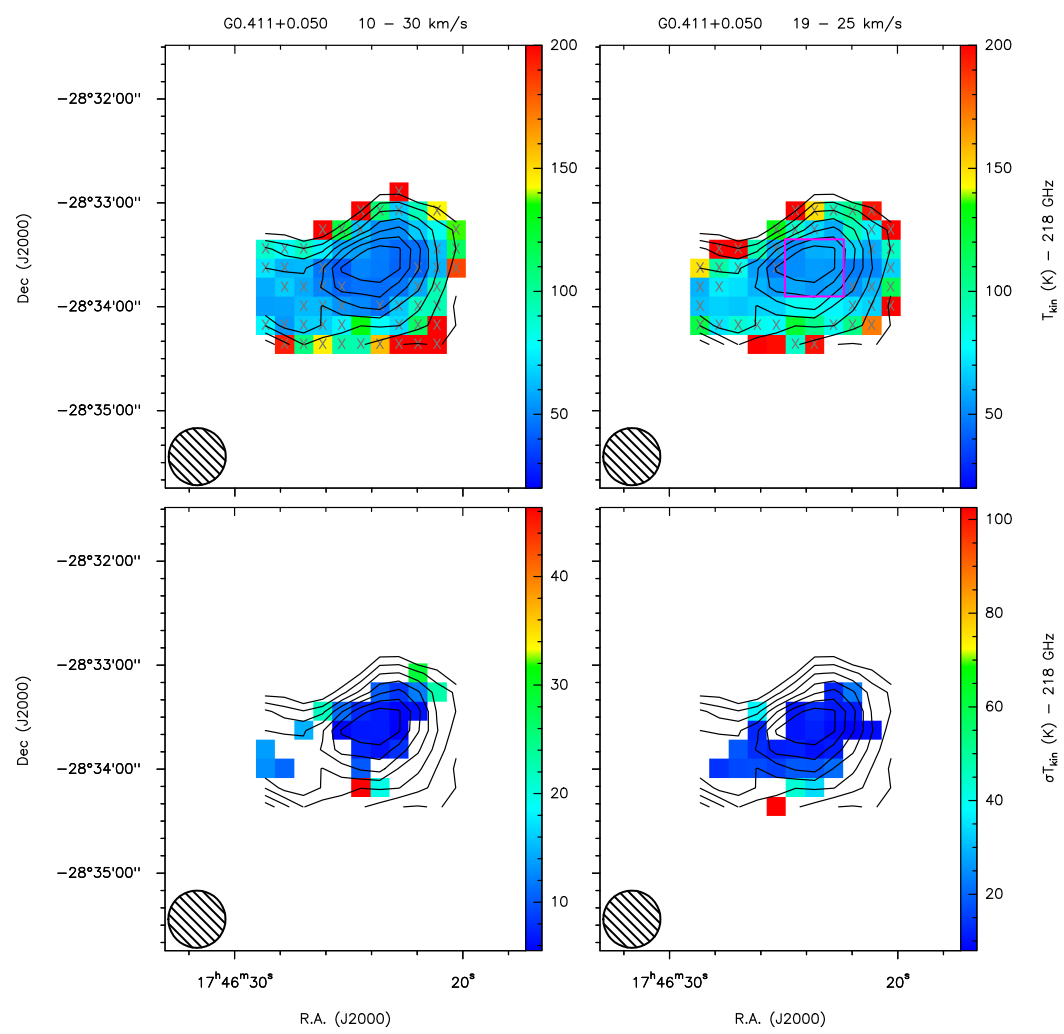

$291 \mathrm{GHz}$ temperatures

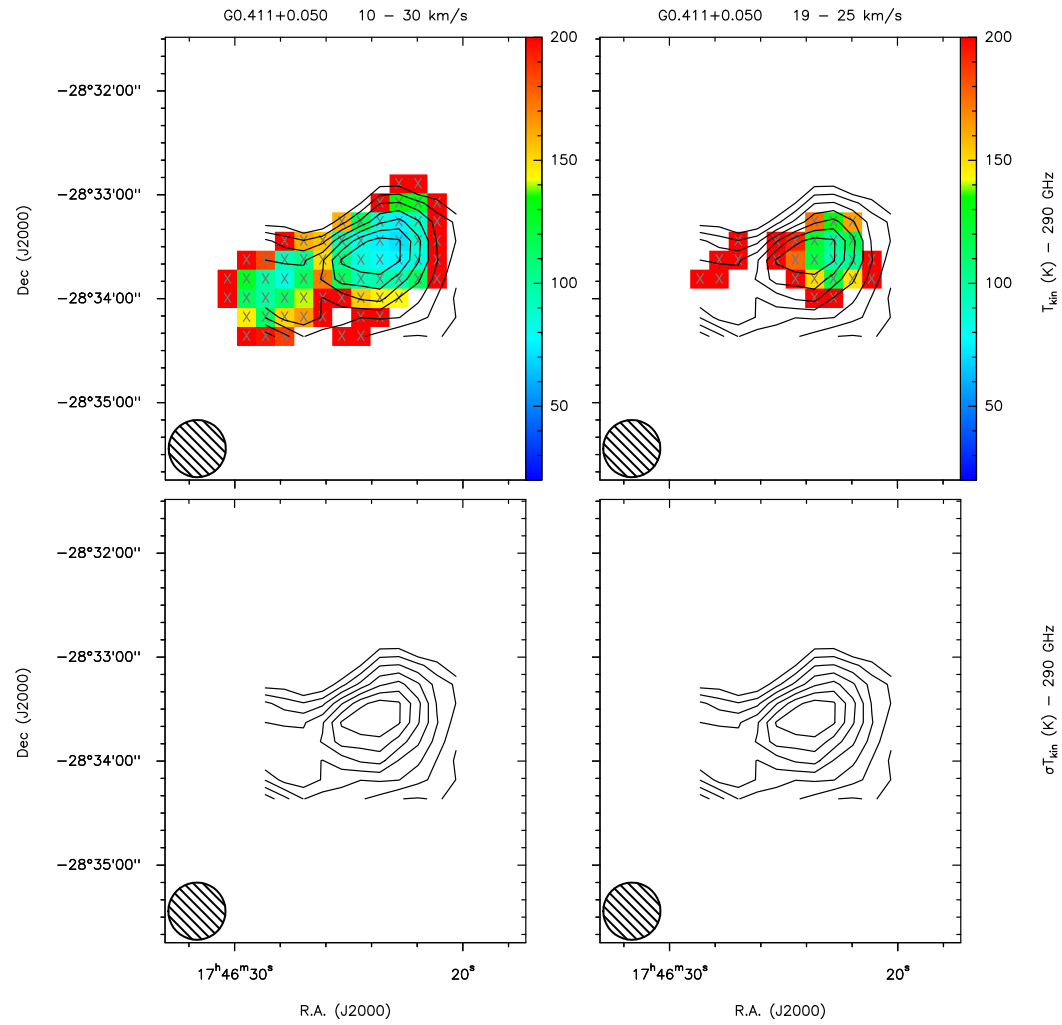

Fig. H.4. As Fig. H.1, for G0.411+0.050. 
K. Immer et al.: Temperature structures in Galactic center clouds

$218 \mathrm{GHz}$ temperatures

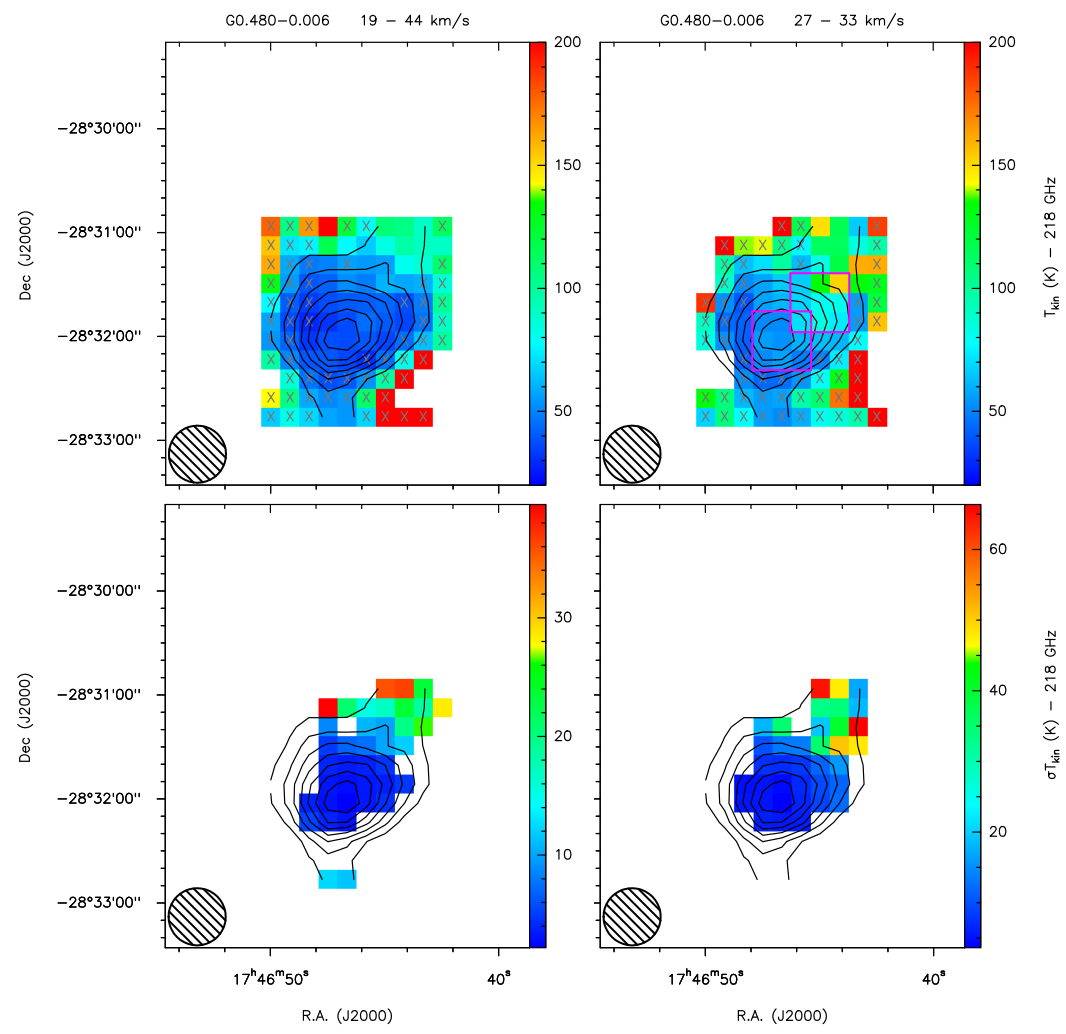

$291 \mathrm{GHz}$ temperatures

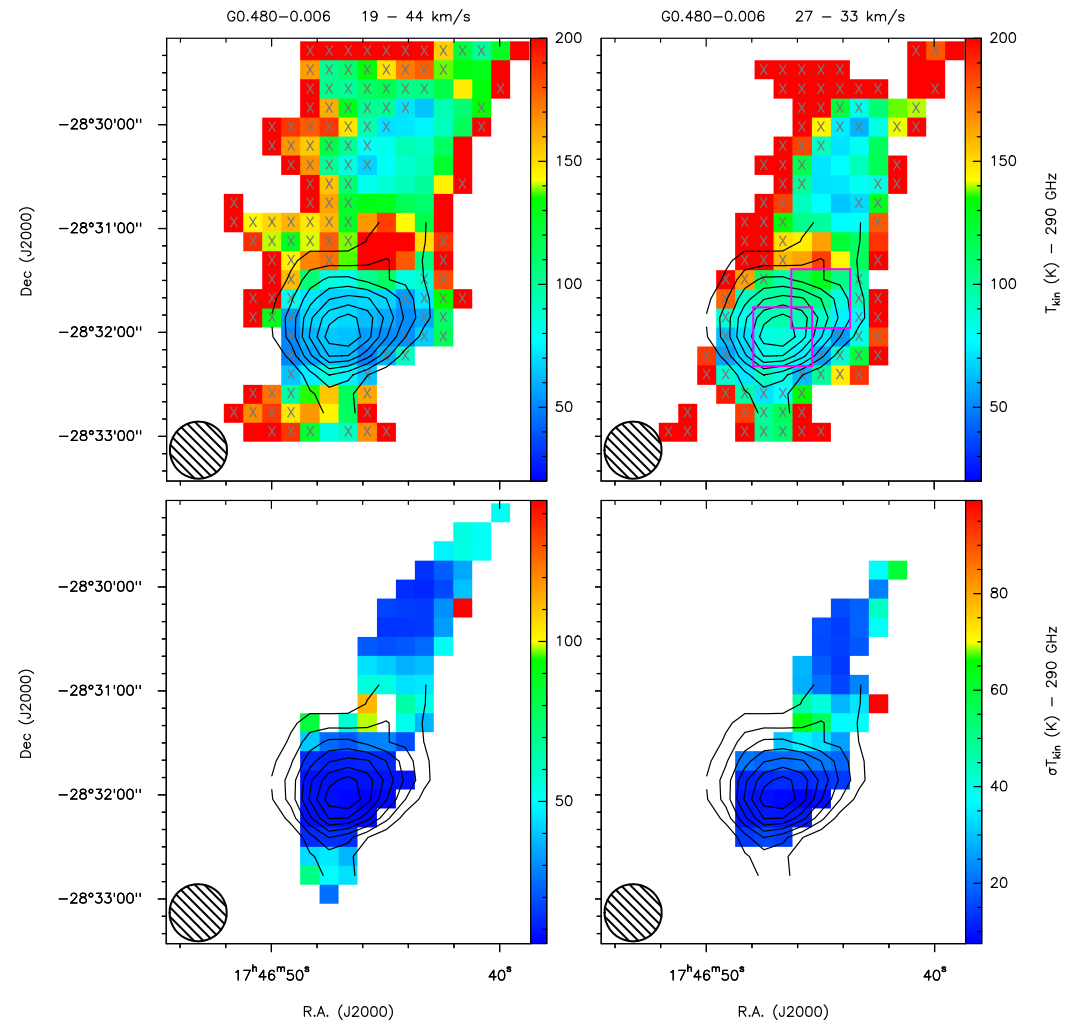

Fig. H.5. As Fig. H.1, for G0.480-0.006. 
A\&A 595, A94 (2016)

$291 \mathrm{GHz}$ temperatures
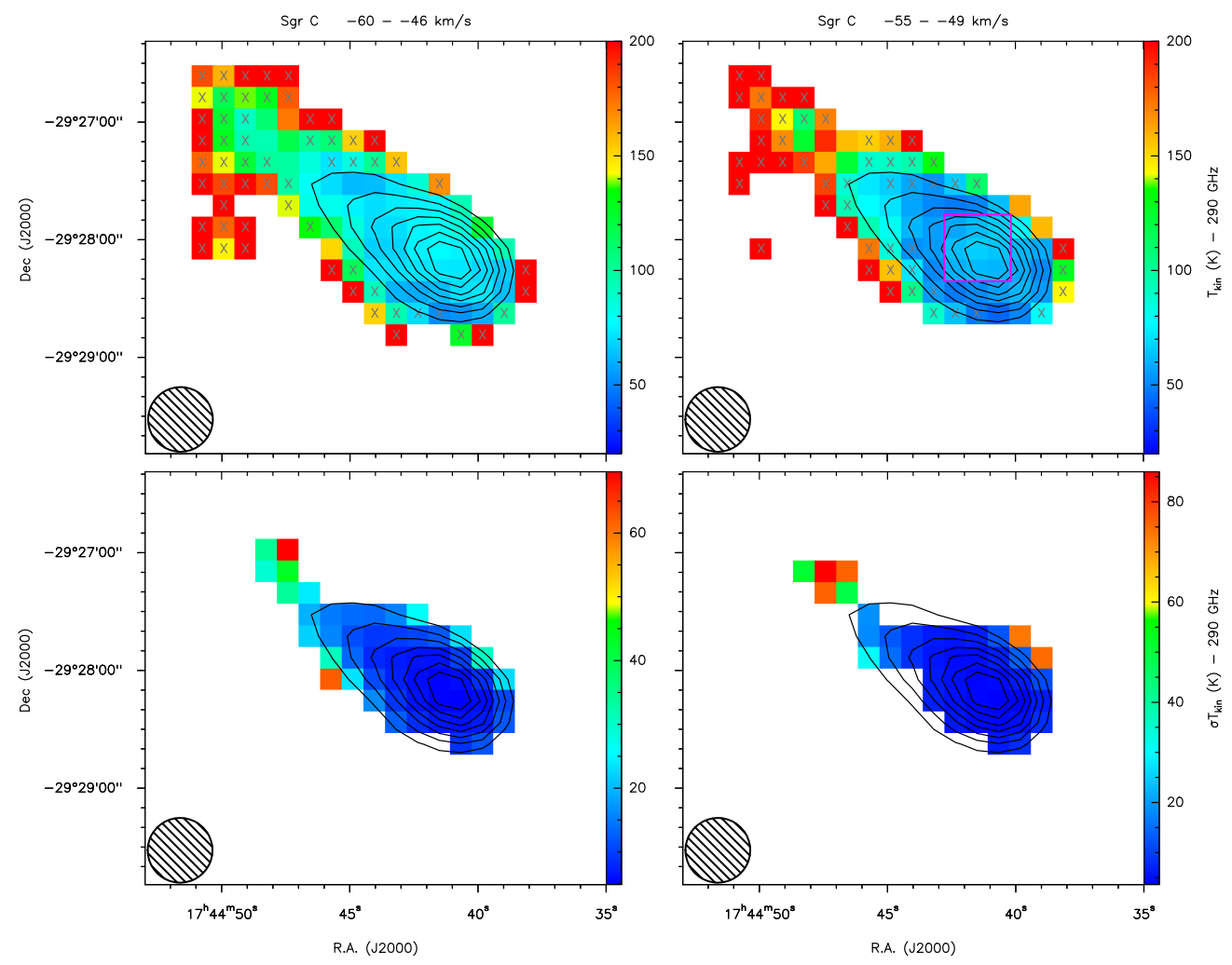

Fig. H.6. As Fig. H.1, for Sgr C.

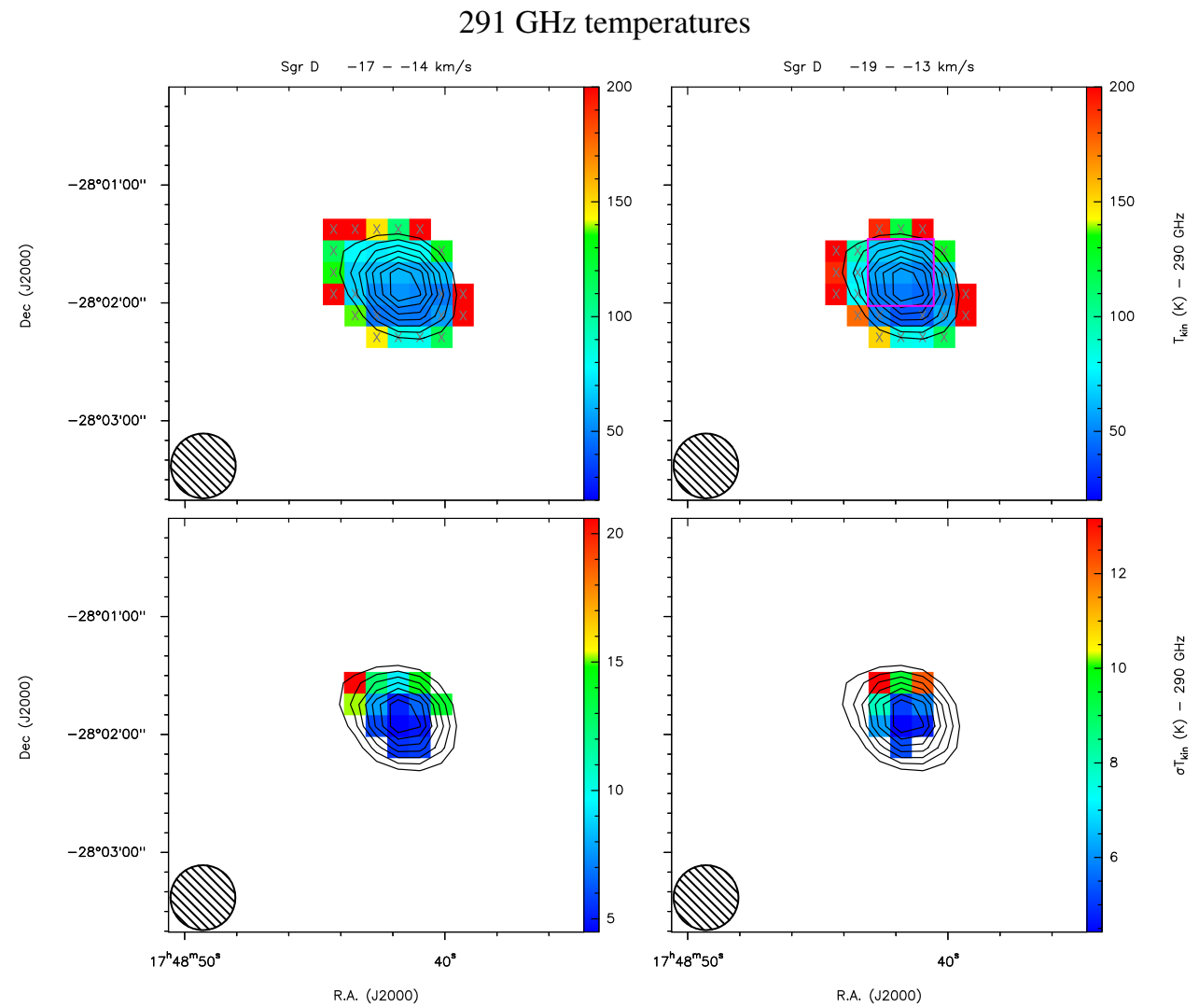

Fig. H.7. As Fig. H.1, for Sgr D. 
K. Immer et al.: Temperature structures in Galactic center clouds
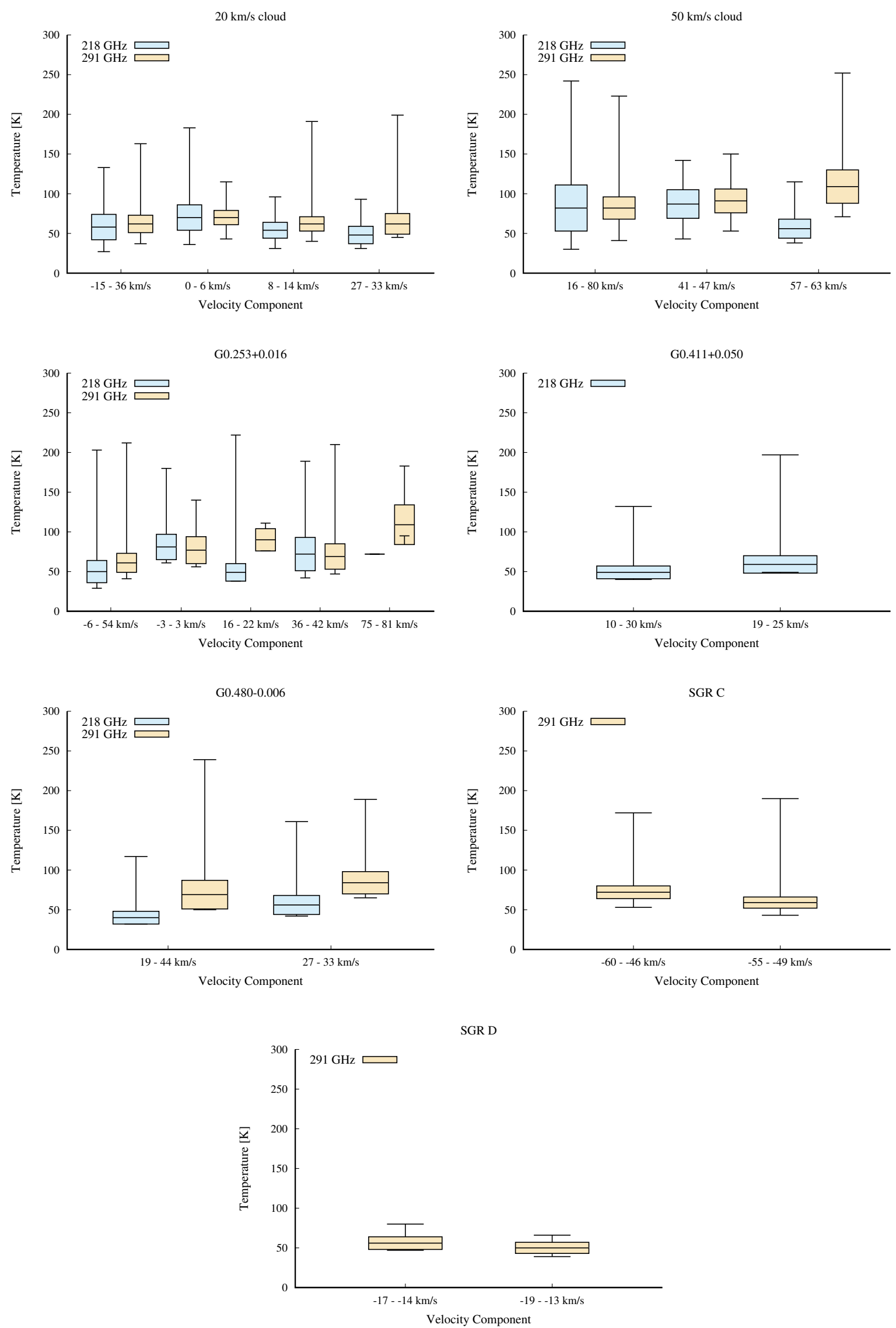

Fig. H.8. Minimal, weighted average, and maximal temperatures of the temperature maps of our seven sources. The vertical size of the boxes is given by the weighted average of the temperature $\pm 1 \sigma$ uncertainty. The values are taken from Table 3 . Blue and yellow boxes show the results at 218 and $291 \mathrm{GHz}$, respectively. 
A\&A 595, A94 (2016)

\section{Appendix I: Temperature vs. line width}

Table I.1. Line ratios, temperatures, and line widths of Fig. 5 at 218 and $291 \mathrm{GHz}$.

\begin{tabular}{|c|c|c|c|c|c|c|c|}
\hline \multirow[t]{2}{*}{ Source } & \multirow{2}{*}{$\begin{array}{l}\text { Velocity component } \\
\qquad\left(\mathrm{km} \mathrm{s}^{-1}\right)\end{array}$} & \multicolumn{3}{|c|}{$218 \mathrm{GHz}$} & \multicolumn{3}{|c|}{$291 \mathrm{GHz}$} \\
\hline & & $R_{321, \text { Average }}$ & $\begin{array}{c}T_{\text {Average }} \\
(\mathrm{K})\end{array}$ & $\begin{array}{c}\text { Line width } \\
\left(\mathrm{km} \mathrm{s}^{-1}\right)\end{array}$ & $R_{422, \text { Average }}$ & $\begin{array}{c}T_{\text {Average }} \\
(\mathrm{K})\end{array}$ & $\begin{array}{c}\text { Line width } \\
\left(\mathrm{km} \mathrm{s}^{-1}\right)\end{array}$ \\
\hline \multirow{5}{*}{$50 \mathrm{~km} \mathrm{~s}^{-1}$ cloud } & \multirow{2}{*}{$41-47$} & 0.409 & 104.9 & 24.6 & 0.464 & 113.4 & 20.9 \\
\hline & & 0.346 & 82.0 & 26.4 & 0.38 & 82.5 & 23.1 \\
\hline & \multirow{2}{*}{$57-63$} & 0.279 & 61.7 & 10.1 & 0.440 & 103.2 & 20.5 \\
\hline & & 0.208 & 45.5 & 14.1 & 0.489 & 126.4 & 17.8 \\
\hline & $-3-3$ & 0.355 & 83.0 & 18.4 & & & \\
\hline \multirow{5}{*}{ G0.253+0.016 } & \multirow{3}{*}{$16-22$} & 0.227 & 57.8 & 19.4 & & & \\
\hline & & 0.198 & 43.7 & 11.6 & & & \\
\hline & & 0.295 & 63.8 & 12.8 & 0.362 & 73.5 & 11.2 \\
\hline & \multirow[t]{2}{*}{$36-42$} & 0.458 & 67.4 & 11.7 & 0.264 & 53.5 & 10.6 \\
\hline & & 0.460 & 131.6 & 19.2 & 0.392 & 87.1 & 17.9 \\
\hline $\mathrm{G} 0.411+0.050$ & $19-25$ & 0.255 & 55.6 & 7.3 & & & \\
\hline \multirow{2}{*}{ G0.480-0.006 } & \multirow{2}{*}{$27-33$} & 0.333 & 74.9 & 11.6 & 0.416 & 92.9 & 12.3 \\
\hline & & 0.252 & 54.4 & 9.3 & 0.385 & 85.0 & 9.7 \\
\hline Sgr C & $-55--49$ & & & & 0.299 & 62.9 & 8.2 \\
\hline Sgr D & $-19--13$ & & & & 0.250 & 51.2 & 4.0 \\
\hline
\end{tabular}

